University of Louisville

ThinkIR: The University of Louisville's Institutional Repository

Electronic Theses and Dissertations

$12-2019$

\title{
Understanding worry and mindfulness through psycholinguistics.
}

\author{
Elena Maria Clara Geronimi Bortoleto \\ University of Louisville
}

Follow this and additional works at: https://ir.library.louisville.edu/etd

Part of the Clinical Psychology Commons

\section{Recommended Citation}

Bortoleto, Elena Maria Clara Geronimi, "Understanding worry and mindfulness through psycholinguistics." (2019). Electronic Theses and Dissertations. Paper 3328.

https://doi.org/10.18297/etd/3328

This Doctoral Dissertation is brought to you for free and open access by ThinkIR: The University of Louisville's Institutional Repository. It has been accepted for inclusion in Electronic Theses and Dissertations by an authorized administrator of ThinkIR: The University of Louisville's Institutional Repository. This title appears here courtesy of the author, who has retained all other copyrights. For more information, please contact thinkir@louisville.edu. 


\title{
UNDERSTANDING WORRY AND MINDFULNESS THROUGH PSYCHOLINGUISTICS
}

\author{
By \\ Elena Maria Clara Geronimi Bortoleto \\ B.A., University of Rio de Janeiro, 2004 \\ B.A., University of Louisville, 2011 \\ M.A., University of Louisville, 2014

\begin{abstract}
A Dissertation
Submitted to the Faculty of the

College of Arts and Sciences of the University of Louisville

in Partial Fulfillment of the Requirements

for the Degree of
\end{abstract}

Doctor of Philosophy in Clinical Psychology

Department of Psychological \& Brain Sciences

University of Louisville

Louisville, Kentucky

December, 2019 
Copyright 2019 by Elena Maria Clara Geronimi Bortoleto

All rights reserved 

UNDERSTANDING WORRY AND MINDFULNESS THROUGH PSYCHOLINGUISTICS

By

Elena Maria Clara Geronimi Bortoleto

B.A., University of Rio de Janeiro, 2004

B.A., University of Louisville, 2011

M.A., University of Louisville, 2015

A Dissertation Approved on

April $15^{\text {th }}, 2019$

by the following Dissertation Committee

Dissertation Director

Janet Woodruff-Borden, Ph.D.

Paul Salmon, Ph.D.

Richard Lewine, Ph.D.

Marci DeCaro, Ph.D.

Kathryn Barten Whitmore, Ph.D. 


\section{DEDICATION}

This dissertation is dedicated to my family; words do not allow me to transcribe my feelings and gratitude. Laura and Rafael have provided me continuous immensurable support and inspiration throughout this journey. 


\section{ACKNOWLEDGEMENTS}

I would like to thank my mentor, Dr. Janet Woodruff-Borden, for her constant guidance, and conceptual expertise throughout this process. I would also like to thank the other committee members, Dr. Paul Salmon, Dr. Richard Lewine, Dr. Marci DeCaro, and Dr. Kathryn Barten Whitmore, for their invaluable insight over the past four years and throughout the completion of this dissertation. I would also like to express my deepest gratitude to my parents for having fostered in me, since my early years, a desire for learning. 


\section{ABSTRACT \\ UNDERSTANDING WORRY AND MINDFULNESS THROUGH PSYCHOLINGUISTICS

\author{
Elena Maria Clara Geronimi Bortoleto
}

April 15, 2019

Our understanding of psychological constructs through language has increased over the last few decades. However, only a few studies have explored linguistic features associated with worry and mindfulness. This is the first study using linguistics to investigate features of worry models associated with worry and mindfulness concomitantly. The current study reviews literatures regarding worry and mindfulness emotional, physiological, and cognitive features, including language. Next, the current study tested the hypotheses that excessive worry would be negatively correlated with present tense and positively correlated with number of words, number of questions, anxiety related words, and negative emotion words, while mindfulness would be positively correlated with present tense and negatively correlated with number of words, number of questions, anxiety related words, and negative emotion words. Further, the hypotheses that worry writing activity would be higher than neutral writing activity in number of words, number of questions, anxiety related words, and negative emotion words, and that worry writing activity would be lower than the neutral writing activity in present tense were also tested. In order to evaluate these hypotheses, 46 predominantly European American women were recruited from the community to complete self-report 
questionnaires and worry and neutral writing tasks. Results of the present investigation suggest that word categories related to emotions (i.e., anxiety and negative emotion words) hold associations with worry and mindfulness. Number of words, questions, and present tense did not reveal an association with worry and mindfulness levels in the present sample. Further, compared to a neutral task, worry activity had higher use of present tense, anxiety-related words, and negative emotion words. The findings may reflect avoidance of emotional content in those higher in worry and lower in mindfulness and acceptance of emotional experience by those lower in worry and higher in mindfulness. Future research should continue exploring linguistic features of worry and mindfulness in order to establish the relevant linguistic variables associated with these constructs, and, concomitantly, explore their clinical applications for treatment and assessment. 


\section{TABLE OF CONTENTS}

PAGE

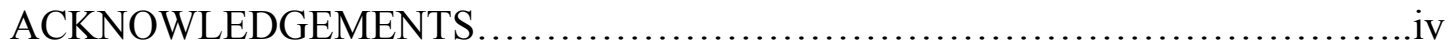

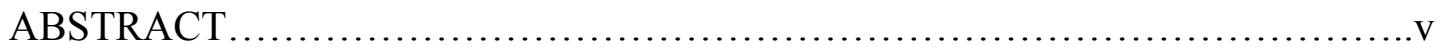

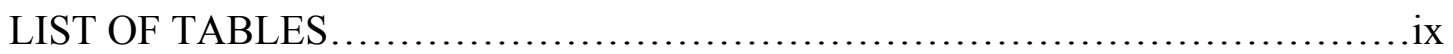

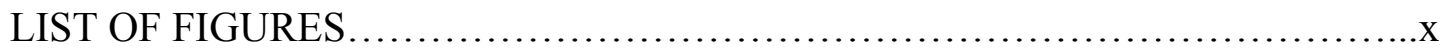

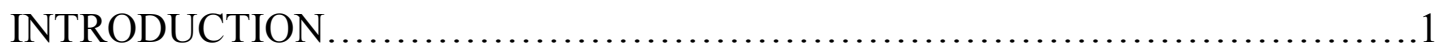

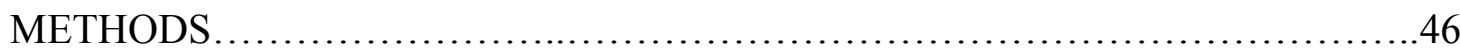

Power Analysis............................................................

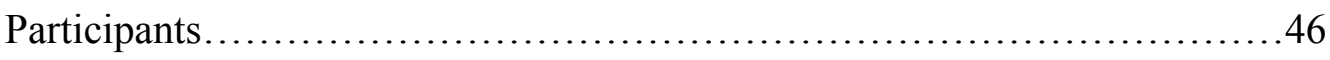

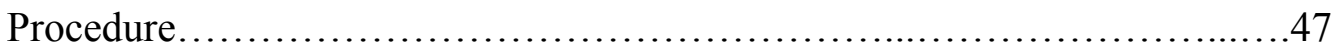

Measures............................................................... 47

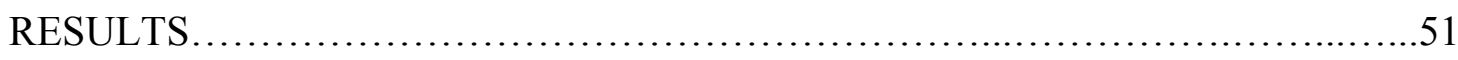

Preliminary Analyses..........................................................

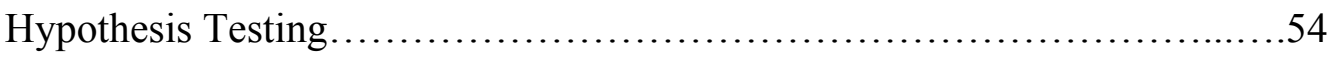

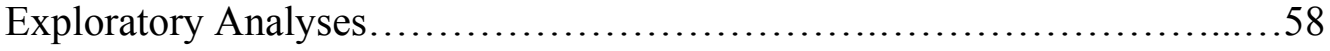

Additional Exploratory Analysis..........................................59

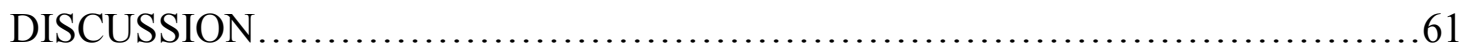

Preliminary Analyses......................................................

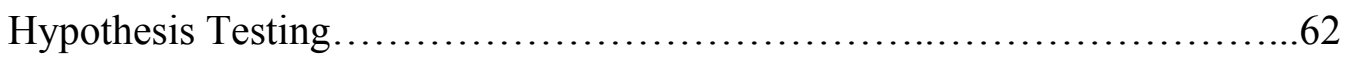

Additional Exploratory Analyses.............................................69

Implications of Linguistic Features for Worry and Mindfulness 
Conceptualizations and Treatment Development.

69

Limitations and Future Research......................................71

Conclusion....................................................... 73

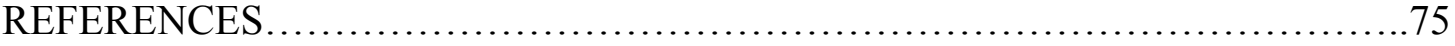

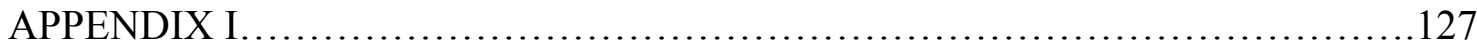

CURRICULUM VITA..................................................... 130 


\section{LIST OF TABLES}

TABLE PAGE

1. Participant Demographics................................................. 91

2. Descriptive Statistics of Self-Report and Language Measures................992

3. Correlation Coefficients for Worry and Mindfulness Variables ..............93

4. Hierarchical Multiple Regression Predicting Worry from Language Variables

5. Hierarchical Multiple Regression Predicting Nonreactivity from Language

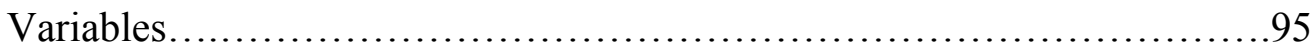

6. Hierarchical Multiple Regression Predicting Nonjudgement from Language

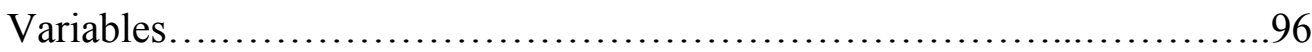




\section{LIST OF FIGURES}

FIGURE

PAGE

1. Worry and mindfulness features.................................... 97

2. Linguistic elements contributing to the "experiential barrier".................98

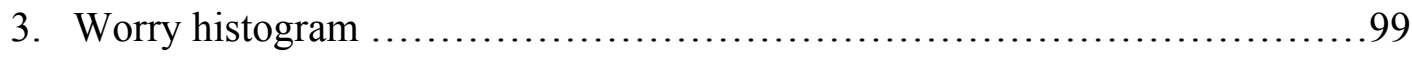

4. Mindfulness histogram........................................... 100

5. Present tense - worry condition histogram............................ 101

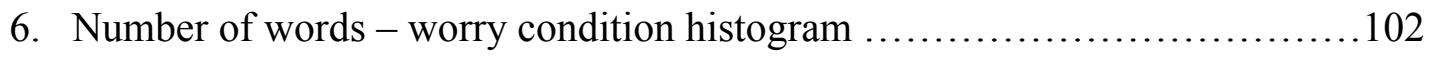

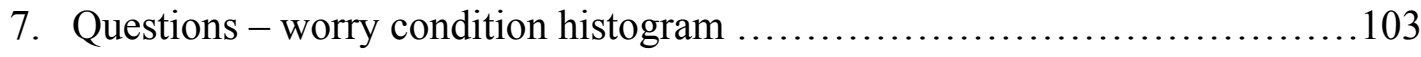

8. Anxiety related words - worry condition histogram .................... 104

9. Negative emotion words - worry condition histogram .................... 105

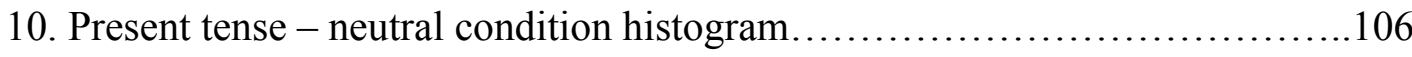

11. Number of words - neutral condition histogram ........................ 107

12. Number of questions - neutral condition histogram...................... 108

13. Anxiety words - neutral condition histogram.........................................109

14. Negative emotion words - neutral condition histogram..................110

15. Worry and language scatterplotts - worry condition.................... 111

16. Mindfulness and language scatterplots - worry condition..................112

17. Worry and language scatterplots - neutral condition...................... 113

18. Mindfulness and language scatterplots - neutral condition..................114

19. Worry Q-Q plot................................................ 115 
20. Mindfulness Q-Q plot ..........................................116

21. Present tense - worry condition Q-Q plot.............................. 117

22. Number of words - worry condition Q-Q plot.........................118

23. Questions - worry condition Q-Q plot...............................119

24. Anxiety related words - worry condition Q-Q plot........................ 120

25. Negative emotion words - worry condition Q-Q plot.....................121

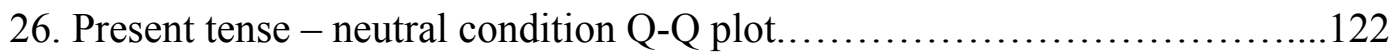

27. Number of words - neutral condition Q-Q plot....................... 123

28. Number of questions - neutral condition Q-Q plot........................124

29. Anxiety related words - neutral condition Q-Q plot.................... 125

30. Negative emotion words - neutral condition Q-Q plot....................126 


\section{INTRODUCTION}

Generalized Anxiety Disorder (GAD) has a high prevalence rate; approximately $5 \%$

of people will develop GAD during their lifetime (APA, 2013). The fundamental feature of GAD is exaggerated, chronic worry that is accompanied by other cognitive and physical symptoms such as sleep problems, difficulty concentrating, and fatigue (APA, 2013). Previous studies have shown that those with GAD experience functional and psychosocial difficulties, are more likely to need heath care services (e.g., Greenberg et al., 1999; Wittchen, Zhao, Kessler, \& Eaton, 1994), and experience low levels of quality of life (Henning, Turk, Mennin, Fresco, \& Heimberg, 2007).

Several theoretical models of worry have been proposed that focus on its prominent emotional, physiological, and cognitive features (see Behar, DiMarco, Hekler, Mohlman, \& Staples, 2009 for a detailed review of theoretical models of worry). These models suggest that worry presents as a barrier to fully experiencing the present moment; that is, worry works as an experiential buffer that prevents the experience of intense emotions and physiological arousal. The difficulty in accepting present experience in pathological worry, and consequently the use of maladaptive strategies (physiological, emotional, and cognitive; Buhr \& Dugas, 2002; Mennin, Heimberg, Turk, \& Fresco, 2002; Thayer, Friedman, \& Borkovec, 1996) are key in perpetuating the worry cycle. In particular, physiological, emotional, and cognitive factors seem to interact in establishing 
this barrier that creates distance from immediate experiences, especially those encompassing negative emotions (Behar, Zuellig, \& Borkovec, 2005). More specifically, in worrisome states, feared stimuli are avoided in the present moment through the use of verbal cognitive activity which has been associated with lower autonomic modulation and poorer emotional processing (Borkovec, 1994). According to the Avoidance Model of Worry, worry is primarily a verbal sequence of thoughts that prevents imagery content from entering consciousness, and thus buffers autonomic arousal (Borkovec, 1994). However, despite the fact that innumerous studies provide support to its verbal nature (e.g., Behar et al., 2005; Borkovec \& Inz, 1990), very little is known about the specific verbal patterns associated with worry. Given that language provides subtle cues about cognitive and emotional patterns, psycholinguistics could provide important insights into the worry activity and broaden our understanding of this phenomenon. However, to date, only two studies examined psycholinguistic features in individuals with GAD and worry activity (Geronimi \& Woodruff-Borden, 2015; Molina, Borkovec, Peasley, \& Person, 1998).

Current conceptualizations of worry stand in direct contrast with the construct of mindfulness which has been defined as an attitude of non-judgmentally focusing attention and being aware of internal and external aspects of the present moment without engaging in further elaborations or reactions (Chambers, Lo, \& Allen, 2008; Kabat-Zinn et al., 1992) which contrasts with the future orientation characteristic of worrisome states as well as negative judgments made about internal experiences while worrying (Geronimi \& Woodruff-Borden, 2015; Wells, 1999). Thus, a mindful state enables one to fully experience the present moment. This ability to be present with whatever arises at a given 
point contrasts with the difficulty in fully engaging in present moment experiences in worrisome states; indeed, mindfulness has been found to be an effective intervention in treating worry (e.g., Craigie, Rees, Marsh, \& Nathan, 2008; Kabat-Zinn et al., 1992). Specific physiological, emotional, and cognitive patterns have been associated with mindfulness; such patterns are antagonistic in relation to those found in worry. For instance, mindfulness states are characterized by more flexible physiological patterns and a better ability to regulate emotions (Feldman, Hayes, Kumar, Greeson, \& Laurenceau, 2007; Vlemincx, Vigo, Vansteenwegen, Van den Bergh, \& Van Diest, 2013).

As such, mindfulness could be used as a way to explain and understand maladaptive patterns that occur during worry, given that, in relation to worry, it presents as a contrasting state where elements constituting worry states (e.g., emotion and physiology) present in an adaptive, balanced form. Indeed, alterations in these domains have been identified as mechanisms of change in mindfulness interventions for worry (e.g., Delgado-Pastor et al., 2015; Delgado et al., 2010; Erisman \& Roemer, 2010). However, less is known about cognitive mindfulness features that could be effective in treating worry (e.g., the internal cognitive dialog characteristic of mindful states; mindfulness relation to cognitive factors associated with worry, such as intolerance of uncertainty - IU, described below). Understanding the psycholinguistic patterns associated with mindfulness could be particularly important in understanding such features. However, to date only two studies have explored linguistic patterns associated with mindfulness (e.g., Collins et al., 2009; Moore \& Brody, 2009).

Given the verbal nature of worry (e.g., Behar et al., 2005) and the immediate access it provides into psychological states (Rude, Gortner, \& Pennebaker, 2004; Stirman 
\& Pennebaker, 2001), linguistics presents the ideal tool for investigating the internal dialog characteristic of worried and mindful states. As aforementioned, several features of worry and mindfulness are contrasting (e.g., physiological, emotional; Feldman et al., 2007; Mennin et al., 2002; Thayer et al., 1996; Vlemincx et al., 2013), and understanding how these features are manifested linguistically will be important in elucidating these constructs and in informing mindfulness interventions for worry. Similar to other areas of psychopathology, in which language has been important in understanding cognitive processes (e.g., depression; Rude et al., 2004), exploring how worry and mindfulness are manifested linguistically will be essential in better understanding these constructs cognitively. Furthermore, as mindfulness presents a distinct, healthier psychological state in relation to worry, establishing how these constructs differ linguistically will be important in delineating the possible cognitive shifts necessary for one to transition from a worrisome to a mindfulness state. Thus, the present study aims to explore the linguistics associated with both worry and mindfulness, as it relates to worrisome states, in order to establish a better understanding of cognitive aspects of worry, especially the internal dialog that takes place during this state. This study will also support and expand on findings about the contrasting nature of worry and mindfulness in other areas, such as emotion and physiology (Feldman et al., 2007; Mennin et al., 2002; Thayer et al., 1996; Vlemincx et al., 2013). In addition, similar to intervention research in these areas (e.g., Delgado et al., 2010), this study will provide an empirical foundation for identifying possible cognitive mechanisms of change in mindfulness-based interventions for worry. Given that GAD is defined by excessive and uncontrollable worry (APA, 2013), not only the worry literature, but also relevant literature related to GAD will be reviewed. 


\section{Worry and GAD: Verbal Nature and Conceptual Models}

\section{The Verbal Nature of Worry}

Worry has been conceptualized primarily as a verbal mental process with a high frequency of language-based thought and low frequency of imagery, a pattern that has been supported in several studies (Behar et al., 2005; Borkovec \& Inz, 1990; Freeston, Dugas, \& Ladouceur, 1996; Hirsch, Hayes, Mathews, Perman, \& Borkovec, 2012). Freeston et al. (1996) found that, when asked to characterize their worry, participants identify a higher frequency of thoughts $(70.4 \%)$ as opposed to images $(24.8 \%)$. In addition, excessive worriers tend to report higher percentages of thoughts compared to low worriers (Freeston et al., 1996).

Using a different method and a clinical sample, Borkovec and Inz (1990) asked participants to engage in worry and relaxation. During relaxation, controls reported higher frequency of imagery over thoughts while individuals with GAD reported equal amount of imagery and thoughts. In addition, during worry, both groups reported higher frequency of thoughts, and after receiving treatment, individuals with GAD reported a decrease in thought activity during relaxation. These findings suggest that thought activity characterizes both worry activity and individuals who have GAD. Hirsch et al. (2012) conducted a similar study; however, they assessed for both frequency and duration of images during worry and thinking about a positive topic. In addition, they also assessed participants more frequently during the tasks. Regarding imagery occurrence, the GAD group reported fewer images than the control group and fewer images occurred when worrying than when thinking about a positive topic. There was also a significant interaction for imagery occurrence, such that fewer images were reported by the GAD 
group during the worry condition but there were no differences in number of images reported during the positive condition. Regarding imagery duration, the GAD group reported briefer images than the control group and briefer images were also reported during worrying than during the positive topic (Hirsch et al., 2012). Further evidence for the verbal nature of worry was found in Behar et al. (2005) study. The intensity in which the induction of worry and trauma recall is associated with imaginal in comparison to verbal activity in both an unselected and a selected sample was investigated. Participants' reports supported the idea that worry is predominantly a verbal thinking process, while trauma recall is an imaginal process. Behar et al. (2005) speculate that during worry activity threatening images are substituted by verbal content as a way of avoiding stronger somatic and emotional reactions (more associated to images) and, thus, maintaining worry through negative reinforcement. Complementing these findings, Hirsch et al. (2015) found that imagery, even negative, reduces later intrusions as compared with verbal perseveration.

In summary, worry is primarily a verbal process, however, to our knowledge, only two studies investigated linguistic features in individuals with GAD and worry (Geronimi \& Woodruff-Borden, 2015; Molina et al., 1998; see details in the "Language: Worry" section, below). These studies suggest that worry and those with GAD are characterized by a unique pattern of language use (e.g., differences in verb tense and connectors). However, more research is needed in understanding the nature of the "internal dialog" characteristic of worry and GAD. Thus, psycholinguistic examinations could contribute to further elucidating the nature of worry. In particular, linguistics could be essential in better integrating the worry models, given that it provides direct and subtle cues about 
cognitive and emotional processes. As a consequence, knowing the language that constitutes worry could provide additional theoretical foundation for treatment studies and interventions.

\section{Worry and GAD Conceptual Models: Physiology, Emotion, and Cognition}

Three main different perspectives (i.e., physiological, emotional, and cognitive) have been adopted in trying to understand the worry phenomenon and GAD (e.g., Buhr \& Dugas, 2002; Mennin et al., 2002; Thayer, et al., 1996), which are associated with specific worry theoretical models (see figure 1). First, from a physiological perspective, worry and GAD have been associated with a restricted range of autonomic responses (e.g., Thayer et al., 1996), which is mostly captured by the Avoidance Worry Model. Second, from an emotional perspective, worry has been characterized by difficulties in emotion regulation (e.g., Mennin et al., 2002), which are the foundation for the Emotion Dysregulation Model of Worry. Third, cognitively, worry and GAD have been associated with a maladaptive pattern of metacognitions (e.g., Thielsch, Andor, \& Ehring, 2015; Wells, 1999) combined with difficulties in tolerating uncertainty (e.g., Buhr \& Dugas, 2002), which are the key features of the IU and Metacognitive Worry Models.

As aforementioned, worry presents as an internal strategy for avoiding present moment experiences (Borkovec, 1994). Despite the fact that the Avoidance Worry Model is the one that most strongly supports worry as an avoidance strategy of present experiences, the other models are also suggestive of the avoidant nature of worry. Indeed, most recently, it has been proposed that none of the worry models alone is sufficient in capturing worry in its entirety, and integrated approaches, incorporating elements of each of the models, as well as their influences on each other, have been more successful in 
explaining worry (Behar et al., 2009). Below, the main worry models are briefly described and literature supporting them is reviewed.

The Avoidance Model. The avoidance function of worry is well established as the fundamental feature of the avoidance worry model; it is also a common feature across other conceptual models of worry (Behar et al., 2009). The avoidance model conceptualizes worry as an avoidant strategy that is reinforced in a variety of ways (Borkovec, 1994). A core feature of this model is the assumption that worry is primarily verbal in nature and that its verbal features are key in preventing the experience of somatic and emotional symptoms that are more closely associated to imagery (Behar et al., 2005; Borkovec \& Inz, 1990). The first empirical attempt to establish worry as a way of preventing the processing of threatening material was conducted by Borkovec and $\mathrm{Hu}$ (1990). Speech anxious females engaged in neutral, relaxation or worrisome thinking just prior to imagining a phobic speech scene. Heart rate (HR) was recorded during the entire procedure and measurements of subjective fear and vividness of the scene were gathered following each trial. Results revealed that, although no differences in HR were found during the three types of thinking, worry caused the greatest subjective fear response to the images and produced the lowest HR responses in the subsequent threatening scene compared to the other two groups. These findings were later replicated (e.g., PeasleyMiklus \& Vrana, 2000).

Taken together, these results suggest that worry may inhibit the processing of threatening information and, thus, maintain the cognitive and affective fear response patterns, despite repeated exposures (Foa, Huppert, \& Cahill, 2006). Complementing the findings related to $\mathrm{HR}$, limited autonomic responses have also characterized worry 
periods as well as GAD participants. More specifically, Thayer et al. (1996) found that, relative to baseline and relaxation periods, worry was associated with heart related measures (e.g., shorter interbeat intervals) that reflect lower autonomic modulation. Similarly, GAD individuals, compared to controls also exhibited poorer autonomic modulation across all tasks (Thayer et al., 1996).

Another way worry is negatively reinforced is by beliefs about worry or functions that are attributed to the worry process. Since the catastrophic outcomes expected by the worrier rarely happen, worry is more likely to reoccur in the future and to be seen as a strategy for preventing undesired outcomes from happening (Borkovec, 1994). Borkovec and Roemer (1995) investigated six perceived functions of worry by individuals with GAD in comparison to controls and nonworried anxious individuals. Interestingly "distraction from more emotional things" distinguished between the three groups, with individuals with GAD scoring higher, followed by nonworried anxious individuals, and controls scoring lower. The authors' interpretation gives further support to the idea that worry serves an emotional avoidant function as well as the suppression of anxiety-related somatic symptoms (Borkovec \& Roemer, 1995). Borkovec (1994) has extensively argued about the avoidance function of worry. According to his view, since the future threat exists only in the minds of the individuals who worry, thought semantic activity is one of the few possibilities left as an attempt to prevent hypothetical negative events from happening.

Despite the fact that clear individual differences exist when attributing reasons to worry, the five reasons most commonly mentioned clearly relate to avoidance of some sort of threat (Borkovec, 1994). Interestingly, the first two (i.e., actual and superficial 
avoidance of catastrophe) are forms of delayed negative reinforcement of worry activity; the other three (i.e., avoidance of emotional content, coping preparation, and motivation) serve as a momentary source of worry reinforcement. Thus, regardless of what the real function of worry may be, it appears to chronic worriers as an "effective" negative reinforcing activity, both in the short and long terms.

The Emotion Dysregulation Model. Emotion dysregulation has been proposed as one of the deficits found in individuals with GAD (Mennin et al., 2002). More specifically, it has been suggested that those with GAD have poor understanding of their emotional states, experience more intense emotions, show negative reactivity to emotional experience, and are not as equipped in regulating their emotions (Mennin et al., 2002; Turk, Heimberg, Luterek, Mennin, \& Fresco, 2005). These difficulties contribute to experiencing emotions as aversive, and thus, those with GAD engage in worry activity to avoid, control, and minimize emotional experiences (Mennin et al., 2002). Similar to the Emotion Dysregulation Model theory, the Contrast Avoidance Model of GAD suggests that those at risk for excessive worry and GAD fear emotional changes from positive or neutral emotions into negative states, using worry to maintain negative emotions and avoid shifts (e.g., Crouch, Lewis, Erickson, \& Newman, 2017; Newman, Llera, Erickson, \& Przeworski, 2014).

Empirical evidence for the role of emotion dysregulation in worry and GAD is provided by several studies (e.g., Mennin, Heimberg, Turk, \& Fresco, 2005; SaltersPedneault, Roemer, Tull, Rucker, \& Mennin, 2006). For instance, Mennin et al. (2005) found that individuals with GAD endorsed more intense emotions, lower ability to understand emotions, higher negative reactivity in response to emotional experience, and 
lower ability to remain calm after experiencing negative emotions in comparison to control individuals. Similarly, Salters-Pedneault et al. (2006) found that poor general emotion regulation was associated with self-reported levels of worry and analogue GAD. In addition, different constituents of emotion regulation such as emotional clarity, acceptance of emotions, and access to effective regulation strategies were negatively associated with worry and analogue GAD.

Additional evidence for the role of emotions in GAD is provided by a study that also investigated worry inductions (McLaughlin, Mennin, \& Farach, 2007). In McLaughlin et al.'s (2007) study, participants with GAD (with and without comorbid dysphoria) were randomly assigned to worry, neutral, and relaxation inductions. After the induction, all participants watched a clip to elicit sadness. Participants with GAD (both with and without dysphoria) in the worry condition experienced higher levels of depressed affect compared to GAD participants in the other conditions and controls. Emotion dysregulation, on the other hand, was higher in those with GAD, but was not affected by the inductions. After viewing the clip, participants with GAD (in both groups) showed lower understanding, acceptance, and management of emotions compared to controls. However, acceptance and management difficulties were higher in those with GAD who also had dysphoria.

In contrast, other studies provide mixed results regarding the association between emotion dysregulation and both GAD and worry. For instance, in one study, those with GAD reported more intense emotional experiences; however, they did not show poor emotion differentiation and applied emotion regulation strategies more often than control individuals (Decker, Turk, Hess, \& Murray, 2008). Analogously, contrary to their 
expectations, Novick-Kline, Turk, Mennin, Hoyt, and Gallagher (2005), found that those with GAD scored higher than controls in emotional awareness. It is important to mention, however, that in this study the method used was not self-report, as in the other studies, but a rater-coded measure.

The specificity of emotion dysregulation to GAD and worry remains unclear, and studies comparing GAD to other pathologies also show mixed findings. Turk et al. (2005) compared features of emotion dysregulation in patients with GAD compared to those with social anxiety. While individuals with social anxiety expressed positive emotions to a lower degree, paid less attention to their emotions, and had more difficulties describing emotions compared to those with GAD and controls, GAD patients reported higher emotion intensity and more fear of experiencing depression compared to the other two groups. Furthermore, emotion measures successfully differentiated between the three groups. Mennin, Holaway, Fresco, Moore, \& Heimberg (2007) investigated the specificity of emotional variables to GAD, major depression, and social anxiety. They found that emotional intensity and maladaptive management are particularly associated with GAD as compared with major depression and social anxiety. On the other hand, poor understanding and negative reactivity to emotions predicted major depression and social anxiety when overlap between disorders was considered, but this was not true for GAD.

The Intolerance of Uncertainty Model. IU has been constantly linked to exaggerated worry and has received growing attention in the field (e.g., Buhr \& Dugas, 2002; Buhr \& Dugas, 2006). IU consists of negative responses when facing uncertain and ambiguous situations (Buhr \& Dugas, 2006). IU is a fundamental component of worry; it 
plays a key role in its etiology and maintenance (Buhr \& Dugas, 2002) and has been conceptualized as a central piece in theoretical models of worry and GAD (Dugas, Gagnon, Ladouceur, \& Freeston, 1998). Further, IU has recently been proposed as a mediator of the relation between personality and worry (Yang, Wang, Chen, \& Ding, 2015).

Early studies examining differences between high and low worriers suggested that high worriers display several features that differentiate them from low worriers. For instance, Metzger, Miller, Cohen, Sofka, and Borkovec (1990) conducted a study investigating the role of worry level in cognitive processing differences. Worriers were classified as those who reported $50 \%$ or greater daily worry and nonworriers as those below this level. Participants engaged in a categorization task. The groups did not show differences in their responses when the stimulus was easily perceived as a member or non-member of the category. However, as the stimulus ambiguity level increased in relation to the category, worriers displayed a higher difficulty in processing. These results suggest that in face of uncertainty or ambiguity, higher worriers show a higher difficulty in making decisions. Consistent with these findings, Tallis and Eysenck (1994) reviewed that several studies have shown that, when faced with categorical decisions, worriers need more evidence than non-worriers before they are able to make a decision. In addition, Russell and Davey (1993) found that worry was significantly correlated with evaluating ambiguous situations as threatening. Taken together, these findings suggest that when faced with ambiguous stimuli, high worriers may experience more difficulties since they tend to evaluate them in a negative way. 
In an attempt to test a conceptual worry model, Dugas et al. (1998) found that IU, beliefs about worry, poor problem orientation, and cognitive avoidance discriminated between individuals with GAD and controls. Most importantly, IU was the variable that most contributed to the model. In the proposed model, the presence of IU functions as a fundamental cognitive piece in initiating questions (e.g., "what if") even when a precipitating stimulus is not present (Dugas et al., 1998). Ladouceur et al. (1999) investigated the specificity of GAD. While sensitivity was found for four process variables (i.e., problem orientation, IU, cognitive avoidance, and beliefs about worry), only IU and problem orientation were variables specific to GAD. Complementary, IU was found to be specific to worry when investigating a nonclinical sample of individuals (Dugas, Gosselin, \& Ladouceur, 2001). More specifically, IU was found to be highly correlated with worry, moderately correlated with obsessions and compulsions, and weakly correlated with panic sensations. In addition, worry was the strongest predictor of IU and IU was the strongest predictor of worry. These findings suggest that the relation between worry and IU is ubiquitous and can be found both in clinical and nonclinical populations.

As a first attempt to investigate the directionality of the relationship between worry and IU, IU was experimentally manipulated (Ladouceur, Gosselin, \& Dugas, 2000). In this study, two groups engaged in a gambling game. One group was told that it was hard to win money for a foundation (increased intolerance) while the other was told that the foundation would get money regardless of the game outcome (decreased intolerance). Results revealed that participants who were exposed to increased intolerance presented higher levels of worry than those who were presented with decreased 
intolerance. Although this study had poor ecological validity and further research is needed before causality can be assumed, it contributes to the understanding of the close relation between IU and worry.

The Metacognitive Model. Another way to conceptualize pathological worry is by the role of metacognitions. Metacognition relates to ideas and evaluations about cognition regarding the capacity to monitor and control cognition (Flavell, 1979; as cited in Wells, 1999). The key piece of this model is that it distinguishes between two types of worry (Wells, 1994, 1995, 1999). Type 1 worry is defined as worry about general events or worry about internal events that are noncognitive in nature; on the other hand, Type 2 worry, or metaworry, is defined in metacognitive terms, or is the worry about the way in which individuals think (Wells, 1994, 1995, 1999).

Based on their experience with the worry processes, individuals with GAD elaborate their own beliefs about worry. Two types of belief are particularly important in this model: positive beliefs about worry, or beliefs that worrying has a protective and coping function, and negative beliefs about worry, or beliefs that worry is difficult to control and can cause threatening consequences (Wells, 1999, 2006). As patients with GAD encounter a threatening situation, positive beliefs about worry are elicited and the individual engages in worry as a coping strategy in order to further evaluate the situation (Type 1 worry).

Positive beliefs about worrying are a frequently used strategy and do not necessarily indicate pathology (Wells, 2006). Nonetheless, as the individual engages in it, the worry process itself may become problematic and contribute to further negative evaluations (Wells, 1999). It is precisely the presence of these negative metacognitive 
beliefs, and the related meta-worry that is key to the start and maintenance of GAD (Wells, 2006). At this point, worry itself may start to be overwhelming and distressing, contributing to negative beliefs about it, or negative metaworry (Type 2 worry). Anxiety then increases as a result of type 2 worry and as a consequence type 2 worry contributes to the view of worry as a threatening process, which the client is unable to deal with. Individuals with GAD respond to type 2 worry in ways that reinforce the negative beliefs and type 2 worry itself. For instance, as individuals with GAD try to control or eliminate the worry experience, they paradoxically experience more worry and higher inability to control it.

Empirical evidence supporting the metacognitive worry model is derived from different sources. First, studies provide support to the association of worry activity in clinical and non-clinical samples with positive beliefs about worry. For instance, Tallis, Davey and Capuzzo (1994) conducted a preliminary study on the phenomenology of worry in a non-clinical sample. They identified two factors associated with the perceived advantages of engaging in worry: motivation and preparatory or analytical thinking. These aspects are closely related to the positive beliefs about worrying. Borkovec and Roemer (1995) investigated positive beliefs about worry in relation to GAD, other anxious states, and control individuals. Participants with GAD rated distraction from more emotional thoughts higher than either the nonworried anxious or control subjects, and they rated superstition and problem-solving higher than the control group (Borkovec \& Roemer, 1995).

Further evidence for the role of positive beliefs about worry in relation to the pathological worry process comes from a study by Cartwright-Hatton and Wells (1997). 
In their study, positive beliefs about worry were a reliable factor in predicting trait anxiety and general worry proneness. Pathological worry was also associated with negative beliefs about uncontrollability and danger, lack of cognitive confidence, and trait anxiety. However, it is important to mention that positive beliefs contributed to worry independently of negative beliefs. According to the authors, the study results are consistent with the idea that some individuals may be more likely to worry because of the positive beliefs they endorse about worrying.

Cartwright-Hatton and Wells (1997) were also interested in testing whether beliefs about worry were specific to individuals with GAD and compared metacognitive subscales of individuals with GAD to those of individuals with Obsessive-Compulsive Disorder, a disorder associated with high worry levels, and with patients with other emotional disorders. According to the findings, individuals with GAD presented similar levels of positive beliefs in relation to individuals with Obsessive-Compulsive Disorder and other emotional disorders, suggesting that although associated with high worry levels, positive beliefs about worry are not specific to patients with GAD. Overall, these findings suggest that individuals who worry, either at a clinical and non-clinical level, present with positive beliefs about worrying. These findings are consistent with the metacognitive model which proposes that beliefs about the advantages of worrying can be conceptualized as a coping mechanism (Wells, 2006). As expected, positive beliefs about worrying are not specific to GAD and are present in non-clinical populations (e.g., Tallis et al., 1994) and other disorders (e.g., Cartwright-Hatton \& Wells, 1997). In addition, they were found not to distinguish between individuals with GAD, high worriers without GAD, and control individuals (Ruscio \& Borkovec, 2004), nor between 
those with GAD compared to those with panic disorder, social phobia, and depression (Wells \& Carter, 2001). According to Wells' (2006) metacognitive model, negative beliefs about worry is the element that distinguishes between pathological and nonpathological worry.

The second main source of evidence for the metacognitive model is based on studies that investigated negative metacognitions and meta-worry in relation to worry (Davis \& Valentiner, 2000; Ruscio \& Borkovec, 2004; Thielsch et al., 2015; Wells \& Carter, 1999; Wells \& Carter, 2001; Wells, 2005). Wells and Carter (1999) provided preliminary evidence of the role of meta-worry in predicting worry and worry-related problems. In a series of regressions, they found that meta-worry significantly predicted both pathological worry and worry-related problems even when controlling for type 1 worries, trait anxiety, and the controllability of worrying. As expected, type 1 worry did not predict worry and worry-related problems when controlling for the other variables analyzed. With the aim of replicating laboratory findings to a more ecologically valid context, Thielsch et al. (2015) found that negative metacognitive beliefs predicted everyday worry even when controlling for trait worry and predicted unique variance in worry when intolerance of uncertainty was also included in a model predicting worry.

Research has also been conducted to compare negative beliefs in those with GAD to controls and other diagnostic groups. Those with GAD scored higher on negative beliefs about worry and meta-worry than those with social phobia and non-patients (Wells \& Carter, 2001). Furthermore, these results remained significant after controlling for type 1 worry. However, individuals with GAD did not differ from those with panic disorder when type 1 worry was controlled for. Evidence also exists for similar levels of 
negative beliefs about worry between those with GAD and obsessive-compulsive patients (Cartwright-Hatton \& Wells, 1997). In Cartwright-Hatton and Wells's (1997) study, GAD patients scored similarly to those with obsessive-compulsive disorder in negative beliefs and both scored higher than individuals with emotional disorders and control individuals. Additional evidence for differences in worry and meta-worry, including negative beliefs about worry, between individuals with GAD and other groups (i.e., nonanxious, non-GAD high worriers, somatic anxiety, and non-worried anxious) is shown in studies by Davis and Valentiner (2000), Ruscio and Borkovec (2004), and Wells (2005). Taken together, these findings suggest that negative metacognitions and meta-worry are consistently related with higher levels of worry and pathological worry. However, these beliefs may not be specific to GAD, since they do not distinguish between those with GAD and those with OCD and panic disorder (Cartwright-Hatton \& Wells, 1997; Wells, 2005; Wells \& Carter, 2001).

\section{Mindfulness}

As detailed above, mindfulness is defined as self-regulated awareness toward the present moment, combined with an attitude of orientation to experience, which includes curiosity and acceptance of one's experiences (Bishop et al., 2004). Worry, on the other hand, is future orientated, serves an avoidance strategy (Borkovec, 1994), and is associated with intolerance of uncertainty (Buhr \& Dugas, 2002). In comparison to worry, mindfulness has been associated with different and more adaptive physiology (Vlemincx et al.,, 2013), emotions (Feldman et al., 2007) and cognitive activity (Jankowski \& Holas, 2014; see figure 1). When approaching experiences in a mindful fashion, one is better able to experience the present moment, given that physiological, 
emotional and cognitive aspects are accepted without judgment (Kabat-Zinn, 2013). Indeed, there is evidence to suggest that low levels of mindfulness are associated with excessive or pathological worry (e.g., Ruiz, 2014). Conversely, understanding mindfulness and what constitutes this state can inform us about mental and emotional processes that occur when worry is at its lowest levels, and thus, inform us about what the necessary elements and strategies would be in order to decrease worry. This is consistent with findings that mindfulness interventions are effective in treating worry (e.g., Craigie et al., 2008; Kabat-Zinn et al., 1992). In this section, mindfulness is first defined, from a Buddhist and a clinical perspective, and its association with healthy psychological states is briefly presented. Next, aspects pertaining to the mindfulness physiology, emotion, and cognitions are briefly presented; special attention is given to features that differ from worrisome states. Then mindfulness interventions for worry are presented followed by a discussion about possible mechanisms of change.

The concept of mindfulness originated in Buddhist practices, but in recent years, has been increasingly adopted in Western healthcare settings. According to the Buddhist view, suffering is a product of not being able to appropriately understand reality and it can be overcome by fully contemplating the world as it is (Thera, 1992). The Buddhist practice of mindfulness is defined as a way of quietly focusing on our internal balance: to note and accept compassionately our own thoughts and emotions at any given point, and to try not to engage in this thinking and emotional flow, having a nonjudgmental awareness of one's own thoughts and emotions (Coffman, 2013). As previously mentioned, in Western clinical settings, mindfulness is generally defined by two main features (Chambers, et al., 2008; Kabat-Zinn et al., 1992). First, mindfulness 
encompasses the process of focusing attention and being aware of internal and external aspects of the present moment. Second, this present-moment experience is faced in a nonjudgmental way; that is, in a mindful state, experiences are simply acknowledged and examined without further elaborations or reactions.

Generally, mindfulness practice, such as breathing and attention meditation exercises, has been associated with better psychological states. In fact, mindfulness training has resulted in lower levels of depression, rumination, negative affect, improvements in some executive function domains (e.g., working memory and sustained attention; Chambers et al., 2008), lower levels of anxiety (e.g., Baer, 2003; Hofmann, Sawyer, Witt, \& Oh, 2010), and worry (Craigie et al., 2008; Kabat-Zinn et al., 1992).

\section{Mindfulness and Physiology}

Studies investigating mindfulness and physiology suggest that the physiological features associated with mindfulness are distinct from those associated with worry, and that they show opposite patterns of responding (Vlemincx et al., 2013). For instance, Vlemincx et al. (2013) compared respiratory variability during worry and mindfulness. Participants engaged in both a worry and a mindfulness-induced period. Several markers of respiratory variability distinguished between worry and mindfulness, such that worry was characterized by reduced breathing stability and decreased variability, while mindfulness was associated with higher variability in respiratory rate and higher breathing flexibility. There were several significant limitations of this study, including a predominance of females, a nonanxious sample, and the utilization of only one session of induced worry and mindfulness. Nevertheless, it provides initial evidence that 
physiological markers of worry are distinct from those of mindfulness and that mindfulness appears to be associated with higher physiological flexibility.

In a study using performance on a breathing exercise to measure mindfulness, Burg, Wolf, and Michalak (2012) investigated the degree to which heart rate variability and mindfulness are associated. As expected, mindfulness and heart rate variability were positively correlated, suggesting higher autonomic regulation in those high in this trait. Complementing these findings, May et al. (2016) showed that eliciting mindfulness in a laboratory through a meditation-like format positively affected cardiovascular modulation.

\section{Mindfulness and Emotion Regulation}

Similar to findings regarding physiology, compared to worrisome states (Mennin et al., 2002), mindfulness has been associated with better emotional strategies (Bockman, Ciarrochi, Parker, \& Kashdan, 2017; Coffey, Hartman, \& Fredrickson, 2010; Feldman et al., 2007). Mindfulness represents the ability to acknowledge emotions as momentary "waves" and experience them entirely with no urge to react to them (Kabat-Zinn, 2013). Consistent with this idea, studies have linked mindfulness traits or tendencies and adaptive emotion regulation strategies (e.g., Coffey et al., 2010; Feldman et al., 2007). For instance, Feldman et al. (2007) found that mindfulness and emotion regulation strategies were highly associated. In addition, through their findings, they argued that a mindfulness state goes beyond management of emotions, but it encompasses flexibility, emotional awareness, and the ability to remain calm when emotionally upset. In line with Feldman et al. (2007) findings, through a path analysis, Coffey et al. (2010) found that mindfulness (conceptualized as a two-factor construct incorporating present-centered 
attention and acceptance of experience) fostered understanding or clarity about experience, which in turn improved the ability to handle negative emotions. Using a diary methodology, Bockman et al.'s (2017) study showed that mindfulness was associated with lower negative and higher positive affect and that when mindfulness, suppression, and reappraisal were tested in the same model, mindfulness predicted unique variance in emotional well-being.

\section{Mindfulness and Cognitions}

Although some aspects of mindfulness and worry suggest that they may differ cognitively (e.g., cognitive flexibility, attention; Fox, Dutton, Yates, Georgiou, \& Mouchlianitis, 2015; Kerr, Sacchet, Lazar, Moore, \& Jones, 2013; Ruiz, 2014), very little is known about how mindfulness and worry differ in relation to cognitive features of the worry models (i.e., verbal and imaginal activity; IU), with the exception of metacognitions, which have started to be explored in the mindfulness context.

Jankowski and Holas (2014) recently proposed a mindfulness metacognitive model, which states that, given its very definition, mindfulness can be conceptualized from a metacognitive perspective. That is, it incorporates two levels of cognition: the basic stage which encompasses elements of immediate experience (e.g., perceptions) and a more advanced stage which consists of a global cognitive awareness incorporating elements of the basic stage. According to the proposed model, this higher metacognitive level also includes several other levels of cognition and metacognition. Mindfulness itself represents the highest metacognitive level, and between this level and the most basic one, several others could exist. For instance, judging oneself for something may be a momentary meta-cognitive level. Interestingly, the higher level of metacognition 
proposed by Jankowski and Holas (2014), which incorporates several other levels of cognition, contrasts with the worry metacognitive model (Wells, 1999) in which "worry about worry" becomes the overarching or dominant metacognition.

Although it offers a new and sound way of approaching mindfulness, the metacognitive model proposed by Jankowski and Holas (2014) requires further empirical validation. For instance, a measure for assessing metacognitions in a mindfulness context has still to be developed. Nonetheless, the model presents an innovative way of conceptualizing mindfulness metacognitively. Thus, in addition to the emotional and physiological aspects that characterize worry and mindfulness as distinct in nature, metacognitions could also be one important feature in making this distinction. Physiologically and emotionally, worry is associated with a restricted or rigid style in comparison to mindfulness (Bockman, 2017; Feldman et al., 2007; May et al., 2016; Mennin et al., 2002; Thayer et al., 1996; Vlemincx et al., 2013). Similarly, the metacognitions characteristic of these states would be manifested by a restricted range of metacognitions in worry compared to a broader and more flexible range of metacognitions in mindfulness (Wells, 2006; Jankowski \& Holas, 2014). However, again, the mindfulness metacognitive model still needs further empirical validation.

Linguistic investigations could be a way of better understanding metacognitive manifestations in mindfulness, as well as other aspects that contrast to worry models, such as verbal and imaginal activity, as well as the IU. These investigations could provide foundation for mindfulness interventions for worry, especially regarding cognitive mechanisms of change. 


\section{Mindfulness Treatments for Worry and GAD}

Mindfulness interventions for worry have been successful in clinical patients as well as in the general population (see Querstret \& Cropley, 2013 for a systematic review), and although most studies used self-report measures, preliminary evidence also suggests

that these interventions may ameliorate physiological markers (see Delgado et al., 2010 study, above).

The Mindfulness-Based Stress Reduction (MBSR) program is an 8-week educational intervention for medical patients consisting of training in mindfulness meditation and its use in daily life (Kabat-Zinn et al., 1992; Miller, Fletcher, \& KabatZinn, 1995). Patients are trained in both formal (e.g., sitting meditation, body scan) and informal (e.g., mindfulness eating, stress reactivity) mindfulness practices in which they are required to engage daily during the program. In Kabat-Zinn et al.'s (1992) study, the MBSR program was used to treat patients with GAD and panic disorder. Assessments (self-report and therapists' evaluations) were obtained prior, during the program, at 3month follow-up, and at a 3-year follow-up period (Miller et al., 1995). Patients showed significant declines in anxiety and depressive symptoms during the course of treatment; these declines were maintained at both the 3-month and 3-year follow-ups. Also, no significant outcome differences were found between the patients with GAD and panic disorder. Pre and post-treatment scores were compared with those of patients initially screened to participate, but who did not take part in the study. No differences were found between the patients enrolled in the program that participated and did not participate in the study; this suggests that no bias effect was suffered by those participating and that the improvements obtained by those in the program could generalize to most of those in the 
MBSR who suffered from similar anxiety problems. In addition, this same pattern was maintained at the three year follow-up (Miller et al., 1995). The comparison between those who took part in the study and those who were in the program but did not participate, as well as conducting a three year follow up (Miller et al., 1995), can be seen as strengths of this study. In addition, at the three year follow-up, the majority of the participants also reported still using formal and informal meditation practices in their everyday lives.

Lee et al. (2007) studied treatment effects on a similar sample (i.e., GAD and panic who were currently taking medication). However, they did not use all aspects of the MBSR, but used a Korean stress-management program which had some features in common with it, such as meditation. Half of the subjects participated in this program, and the other half participated in an education program. The meditation program showed significant improvements in anxiety compared to the education group. Changes in depression were mixed, since they varied according to the measure used. Furthermore, those in the meditation group showed more improvement in hostility scores.

Similar to the MBSR, the Mindfulness-Based Cognitive Therapy (MBCT) program involves 8-week 2-hour group sessions (Craigie et al., 2008; Segal, Williams, \& Teasdale, 2012). In addition to mindfulness elements, the MBCT also involves CBT elements such as psychoeducation. Evans et al. (2008) investigated the effectiveness of an open trial 8-week group receiving MBCT. Results revealed a significant decrease in worry, depression, anxiety, and tension after the therapy program. Patients with clinically significant symptoms of anxiety, worry, and depression had their scores reduced to non- 
clinical levels after the program. There was also a decrease in mindful awareness; however this finding failed to reach significance.

Craigie et al.'s (2008) study more extensively evaluated improvements in GAD patients following a group MBCT program. Large treatment effects were found for measures of worry, depression, stress, and fear of relaxation. Improvements were small only for anxiety. At the 6-week follow up, all measures showed significant improvements relative to pre-treatment and similar results were found at the 3-month follow-up, with the exception of anxiety that failed to reach significance. Effect sizes had similar ranges to those observed at post treatment. Post-treatment scores and follow-up points were compared as well. The only significant finding for this analysis was that at the 6-week follow-up, there was an improvement in worry scores. Still regarding worry scores, the majority of patients experienced a reliable improvement at post-treatment; however, only a low percentage of them had recovered from this condition. Improvements on worry and depression measures were similar and slightly better than those found in Evans et al.'s (2008) study. The authors mention, however, that although worry effect sizes were large, they were still lower than those observed in multi-component CBT treatments. A number of factors such as clinical complexity and co-morbidity may account for these results. Patients reported improvements in quality of life, suggesting that MBCT is associated with improvements beyond disorder specific symptoms.

Kim et al. (2009) investigated the effectiveness of a MBCT intervention in patients with GAD or panic disorder currently taking medication. Subjects were assigned to either MBCT or an anxiety disorder education program. The MBCT group had greater decreases in anxiety, obsessive-compulsive, and depression scales compared to the 
education group. In addition, at post-treatment, significantly more patients in the MBCT group met criteria for remission. No differences between groups were found for scales of somatization, interpersonal sensitivity, paranoid ideation, psychoticism, and global sensitivity index. However, this finding is not surprising, given that these scales should in fact not be as closely related to changes in anxiety levels as anxious and depressive scales.

Yet a different mindfulness-based intervention used in patients with GAD is the Acceptance-Based Behavior Therapy (ABBT). Similar to MBCT, ABBT combines elements of both mindfulness and CBT interventions. The avoidance theory of worry, the difficulties with emotions, and behavioral avoidance found in high worriers provide theoretical and empirical support for using ABBT for treating worry and GAD (Roemer \& Orsillo, 2002, 2007). Roemer and Orsillo (2002, 2007) propose an ABBT program based on promoting present-moment awareness, developing acceptance rather than judgment of internal experiences, and encouraging action in areas that the individual finds meaningful. In an open trial of ABBT, GAD clients received individual therapy and were assessed pre-post, and three months after treatment ended (Roemer \& Orsillo, 2007). Results revealed that large and lasting effects were found for GAD severity, worry, anxiety, depressive symptoms, and quality of life. The proportion of clients classified as responders at posttreatment was higher than in previous trials, although the proportion classified as high end-state functioning was equivalent. However, these proportions decreased at the 3-month follow-up, making them either comparable to or slightly lower than previous trials. 
Roemer, Orsillo, and Salters-Pedneault (2008) expanded Roemer and Orsillo's (2007) study. Clients were randomly assigned to immediate or delayed treatment. Treatment had a large effect on GAD-specific outcomes (i.e., GAD severity, worry, and stress) and depressive symptoms. Marginally significant findings with medium effect sizes were found for anxious arousal symptoms, quality of life, and additional diagnoses. In addition, the treatment changes were clinically significant and lasting. At posttreatment, a high percentage of the sample met criteria for responder and high end-state functioning, and these results were maintained at the 9-month follow-up, thus providing better outcomes as compared to Roemer and Orsillo's (2007) study.

Although key in explicating the important role of mindfulness in treating worry and $\mathrm{GAD}$, the aforementioned studies have several limitations; for instance, some of them do not have a worry-specific measure (e.g., Kabat-Zinn et al., 1992, 2009; Lee et al., 2007), do not have a control or comparison group (e.g., Craigie et al., 2008; Evans et al., 2008; Kabat-Zinn et al., 1992; Lee et al., 2007), had patients enrolled in the study who were taking medications, making it hard to delineate the specific contribution of the treatment (e.g., Kim et al., 2009; Lee et al., 2007), and had a small sample size (e.g., Evans et al., 2008).

\section{Possible Mechanisms of Change Involved in Mindfulness Treatments for Worry}

As mentioned above, the worry cycle is maintained through the inability to accept present experience in pathological worry, and consequently the use of maladaptive strategies (physiological, emotional, and cognitive; Bockman, 2017; Buhr \& Dugas, 2002; Feldman et al., 2007; Mennin et al., 2002; Thayer et al., 1996). Contrarily, in a mindful state, the acceptance of whatever is presented at a given moment (Kabat-Zinn, 
2013), even painful situations and experiences, is responsible for the maintenance of a healthier state and for more adaptive physiological, emotional, and cognitive experiences. Again, not surprisingly, mindfulness treatments have been effective in treating worry (Craigie et al., 2008; Kabat-Zinn et al., 1992).

Given this contrasting approach to experience in mindfulness and worrisome states, combined with evidence of the effectiveness of mindfulness in treating worry (Craigie et al., 2008; Kabat-Zinn et al., 1992), it appears that developing a more mindful approach to reality, or engaging in the emotional, physiological, and cognitive patterns that constitute mindful states, is important in decreasing worry symptoms. Indeed, mindfulness itself has been proposed as a mechanism of change in mindfulness interventions for worry (Roemer et al., 2008). Mindfulness is a complex, multicomponent construct, and understanding how each of its components (i.e., physiological, emotional, cognitive) change may better inform us about the changing trajectories involved in worry interventions based on this approach. Literature on the mechanisms of change involved in mindfulness interventions for worry, or to elements of worry models, is reviewed below.

Changing physiology. In the Delgado et al. (2010) study, psychological and physiological outcomes were analyzed after a mindfulness-based intervention for high worriers. A group of female university students participated in a mindfulness intervention or a progressive muscle relaxation plus self-instruction to postpone worrying intervention. The two groups did not differ in clinical and daily self-report measures. However, those participating in the mindfulness intervention reported better emotional comprehension and displayed a reduced breathing pattern and higher vagal reactivity, 
thus showing better somatic and autonomic regulation. Combined, these results suggest that mindfulness contributes to better emotional and physiological strategies as opposed to those associated with pathological worry.

Tang et al. (2009) investigated physiological outcomes during and following an integrative body-mind training based on meditation and mindfulness principles as compared with a relaxation group. The integrative group displayed better physiological markers as measured by heart rate, rate and amplitude of respiration, and skin conductance as compared to the control group, during and following training. That is, comparisons of these physiological markers between groups suggest that the autonomic nervous system was more effective in the integration group then in the control group, thus providing evidence that mindfulness-based interventions are superior to relaxation in improving physiological markers found to be associated with worry in previous studies (e.g., Thayer et al., 1996). Further, a recent study provides evidence of the effectiveness of mindfulness treatments for changing biomarkers in those with GAD (Hoge et al., 2018). Hoge et al. (2018) found that, in comparison to those in an attention control class, MBSR participants had greater decrease in adrenocorticotropic hormone and in inflammatory cytokines concentrations.

Changing emotions. Davidson (2010) proposed that mindfulness interventions may change one's connection to one's own emotions such that they are conceptualized as transient states as opposed to essential constituents of the self. The development of this ability may be particularly important in treating worry, given the difficulties in managing emotions found in high worriers (Mennin et al., 2002). Although several studies suggest emotion pattern modification as mechanisms of change in mindfulness interventions 
(e.g., Arch \& Craske, 2006; Sass, Berenbaum, \& Abrams, 2013), only one study to date investigated changes in emotion in an intervention for high worriers (Delgado et al., 2010). In this study, compared to the control group, those participating in the mindfulness intervention showed higher emotional comprehension, defined as better understanding and ability to distinguish between feelings (see details about this study above).

Sass et al. (2013) used a brief, five session mindfulness training, to test discomfort with emotions as a moderator of mindfulness interventions. Discomfort with emotions, or low emotional acceptance, was found to moderate reductions in distress. That is, those low in discomfort with emotion displayed more reduction in distress as a result of the mindfulness intervention. Evidence of the important role of emotions in mindfulness interventions also comes from a study using a neurological approach to measure emotions. Farb et al. (2010) used functional MRI to investigate neurological differences in response to a sadness induction (i.e., film clips) in participants completing eight weeks of mindfulness training as compared to waiting list controls. Although reports of sadness were not significantly different between the groups, those participating in the mindfulness training displayed a different neural response, such that there was greater right-lateralized activity, corresponding to visceral and somatosensory areas related to body sensation; the intensity of this neural activity following sadness induction was also associated with lower depression ratings.

Additional support to the role of emotions in mindfulness interventions also comes from laboratory settings (Arch \& Craske, 2006; Erisman \& Roemer, 2010). Participants in the Erisman and Roemer (2010) study watched distressing, positive and emotionally ambiguous film clips right after having watched analogous film clips and 
receiving either mindfulness or neutral education (control condition). There were no differences in emotion regulation and response for the two conditions after the distressing film clip. However, participants in the mindfulness intervention condition showed higher levels of positive affect after the positive film as well as better emotion regulation and less negative affect after the emotionally ambiguous clip.

Similarly, in Arch and Craske's (2006) study, a brief mindfulness intervention took place in the laboratory in the form of a breathing induction. Arch and Craske (2006) were interested in the emotion regulation mechanisms of mindfulness. Participants were randomly assigned to the mindfulness induction, unfocused attention, or worrying. Each group viewed a group of positive, neutral, and negative slides prior and after the inductions. While the mindfulness group did not show differential responses to the slides shown prior and after the induction, those in the other two conditions responded more negatively to the neutral slides presented after the inductions as compared to those presented before it. Compared to the worry group, the mindfulness group also had lower negative affect scores and emotional unpredictability following the post-induction images; compared to the unfocused attention group the mindfulness group showed higher disposition to watch negative images. The responses displayed by the mindfulness group show more adaptive responses to negative stimuli and better emotion regulation strategies.

Changing cognitions. To date, no studies have directly explored cognitive elements derived from worry models as potential mechanisms of change in mindfulness interventions for worry; however, results from two related studies suggest that metacognitions could be an important aspect of worry or other psychopathology 
interventions. In this section we first discuss a study that used a metacognitive intervention for treating worry (Delgado-Pastor et al., 2015), then, we present a study that found metacognitive awareness to be a mechanism of change in depression interventions (Teasdale et al., 2002).

Delgado-Pastor et al. (2015) tested effects of two metacognitive constituents of mindfulness: metacognition and metainteroception (awareness of interoceptive sensations) in high worriers. Participants were randomly assigned to three groups: metacognition intervention, metainteroception intervention, and non-intervention. The metainteroception intervention consisted of exercises aimed at paying attention and accepting bodily and emotional states; the metacognition intervention was similar to the metainteroception one, but also incorporated awareness of one's thoughts. Despite this study's limitations which included a female-only sample, small sample size, and short intervention, both clinical groups showed improvements in clinical symptoms and mindfulness. However, the metainteroceptive group was superior in that it also showed improvements in physiological measures of autonomic regulation. It could be argued that metainteroception is also an element of metacognition, given the fact that it refers to internal awareness (Jankowski \& Holas, 2014). Overall, results from this study suggest that interventions targeting metacognitions are effective in treating worry.

Teasdale et al. (2002) investigated the role of metacognitive awareness, a concept closely related to metacognitions, in prevention of relapse in depressive patients exposed to either Cognitive Therapy or MBCT. Results showed that depressive metacognitions were more accessible in controls than in depressed patients and that the degree to which metacognitions were accessible predicted depression relapse. Both cognitive therapy and 
MBCT reduced relapse by increasing accessibility of metacognitions. Thus, results from this study suggest that higher levels of metacognition are associated with healthier states.

Although this research is still very limited, aspects associated with adaptive physiology and emotions, presented above, have been proposed as potential mechanisms of change in mindfulness treatments for worry or for changing deficits related with worry (e.g., poor autonomic regulation, deficits in emotion regulation). Changes in cognitive activity could be another possible mechanism of change in mindfulness treatments for worry, however, compared with physiological and emotional features, little is known about what distinguishes worry and mindfulness cognitively. Establishing these differences will be essential in providing foundation for future studies assessing cognitive mechanisms of change. Linguistics presents as the appropriate method for this aim, given the direct access it provides to cognitions. Thus, through the use of linguistics we could have access to cognitive patterns or the internal dialog characteristic of worry and mindfulness states.

Below, psycholinguistic studies in psychopathology are discussed, particularly those investigating worry and GAD, as well as preliminary evidence involving the language of mindfulness.

\section{Psycholinguistics}

Given the aforementioned verbal nature of worry (e.g., Borkovec \& Inz, 1990; Freeston et al., 1996), investigating the language associated with this mental activity and GAD is warranted in order to better understand this disorder and the structure of worry, which is closely associated with it. This investigation could be effectively accomplished through the use of psycholinguistics, given that psychological states can be assessed by 
the language one uses (e.g., Rude et al., 2004; Stirman \& Pennebaker 2001).

Psycholinguistics is the intersection area between psychology and linguistics (Field, 2005). It concerns the study of several linguistic aspects (i.e., semantics, grammar, phonology) as they relate to psychological aspects such as brain structure, behavior, cognitions, and most recently, psychopathology.

Preliminary evidence suggests that a specific linguistic pattern is associated with worry and GAD (Geronimi \& Woodruff-Borden, 2015; Molina et al., 1998). The exploration of psycholinguistic patterns also presents as an important alternative for addressing the limitations of extant research on worry. For instance, compared to the use of questionnaires, psycholinguistics presents more naturalistic ways of assessing worry features; psycholinguistics could also be helpful in establishing the key features associated with each of the worry models, given the immediate access it provides to psychological states, an area needing further exploration (Behar et al., 2009).

Likewise, psycholinguistics could be essential in the investigation of healthier psychological states, such as mindfulness. Furthermore, given the contrasting nature of worry and mindfulness, the investigation of linguistic features or "internal dialog" associated with mindfulness could be helpful in distinguishing and delineating cognitive patterns associated with less adaptive thought processes, such as worry. Using such methods for studying mindfulness could also be particularly powerful in understanding this construct, given previously raised difficulties in operationally defining this complex concept (Grossman, 2008). Indeed, initial evidence suggests that mindful states may also relate to specific linguistic patterns (e.g., Collins et al., 2009; Moore \& Brody, 2009). 
Most importantly, given the effectiveness of mindfulness interventions for worry (e.g., Craigie et al., 2008; Kabat-Zinn et al., 1992), better defining both constructs - worry and mindfulness - linguistically may tell us about the changes that need to occur cognitively for one to transition between a worrisome to a mindfulness state. That is, it could give us insight into the cognitive mechanisms of change involved in mindfulness interventions for worry. In particular, language as a method of assessing cognitions and emotions gives access to features that are not reachable through the use of self-report methods, which are frequently used in psychopathology investigations. This section briefly delineates the few findings related to the language of worry and mindfulness.

\section{Language: Worry}

Geronimi and Woodruff-Borden (2015) investigated linguistic features of individuals with GAD compared to nonanxious persons. The study examined parental language in a free play interaction designed to mimic a simple, naturalistic, daily situation. More specifically, based on the avoidance and intolerance of uncertainty theoretical models of worry, linguistic elements of parental speech were hypothesized to predict parental diagnostic status. As expected, elements of the avoidance worry model

(i.e., present tense, future tense, and prepositions) and the intolerance of uncertainty model (i.e., number of questions) correctly classified those with GAD versus those who did not meet criteria for an anxiety disorder; that is, a considerable amount of the variance in diagnostic status was explained uniquely by language use. The presence of specific linguistic patterns in the speech and writing of individuals with depression or an anxiety disorder suggest that these individuals may have a particular way to turn their attention and process specific aspects of their environment, which may be driven by 
rooted biases associated with their conditions (e.g., Geronimi \& Woodruff-Borden, 2015; Hofmann, Moore, Gutner, \& Weeks, 2012; Rude et al., 2004).

Using a think-aloud procedure, Molina et al. (1998) evaluated the content and pattern of worrisome process, with the aim of capturing individual's variations in "stream of thought". Participants were characterized as meeting criteria for GAD by questionnaire, dysphoria, or controls. Participants were first instructed to simply thinkaloud (neutral task) and then to worry aloud (worry task). After the worry task, they were asked what their worry was primarily composed by: thoughts, images, or both. Thinkaloud and worry aloud contents were transcribed and coded for several features (e.g., temporal orientation, affect statements) and word search was conducted for some categories (e.g., cognitive distortions and somatic anxiety). In terms of temporal orientation, there was a significant decrease in present-oriented statements from neutral to worry periods and increases for future-oriented and past-oriented statements, although these did not reach significance. For affect statements, the proportion of high positive affect and low negative affect statements decreased from neutral to worry periods, while high negative statements increased. Regarding references to the environment (i.e., references to the laboratory), there was a decrease from neutral to worry periods and the same decrease was present for degree of shifting (i.e., how much participants shifted from one topic to another). In regards to adjectives, combining neutral and worry periods, participants with GAD used a higher frequency of anxiety-related words and dysphoric participants used a higher frequency of the word "worry." Not surprisingly, the word "worry" was used at a higher frequency during worry compared to the neutral periods. There was a tendency for an increase in negative mood adjectives from neutral to worry 
periods in dysphoric participants. Considering cognitive distortions (e.g., should, never, horrible), overall there was a higher frequency of "have to/should" words. There was a higher frequency of cognitive distortions during worry compared to the neutral period. Analogously, participants with GAD showed the highest occurrence of cognitive distortions followed by dysphoric and control participants. There was a lower frequency of "always/never" words among dysphoric participants compared to the other groups, and participants with GAD showed a higher frequency of "awful/terrible/horrible/what if" and "have to/should". Finally, there was a tendency for participants with GAD and dysphoric participants to report a predominance of thoughts during worry as opposed to controls who reported predominantly a mixture of thoughts and images.

Interestingly, Molina et al. (1998) found that present statements were higher during neutral periods as compared to worry, and that future statements were higher during worry. Using a different method, Geronimi and Woodruff-Borden (2015) found that GAD was associated with more future and less present tense use. Combined, these findings suggest that a temporal bias may be characteristic of both those with GAD and worry activity. Further, in the only study to date examining linguistic changes in those with GAD during psychotherapy (i.e., Therapist-Assisted Internet-Delivered CBT), Dirkse, Hadjstavropoulos, Hesser, and Barak (2015) found that past tense words increased during treatment. Dirkse and colleagues (2015) also found that the use of negative emotion, anxiety, causation, and insight words were reduced as treatment progressed and that negative emotion words covaried with symptom ratings.

Overall, studies looking at language features in psychopathology have contributed to a better understanding of cognitions and emotions in specific groups. However, most 
studies were conducted in the laboratory, thus limiting the ecological validity of the findings. More studies on linguistics of psychopathology are needed using more naturalistic methods in order to examine the generalizability of these findings to daily experiences. In addition, some studies focused on written language (e.g., Rude et al., 2004) while others focused on spoken language (e.g., Hofmann et al., 2012); more studies are needed using these different communication forms in order to determine the solidity of the findings.

\section{Language: Mindfulness}

The language associated with mindfulness has only been investigated in two studies related to treatment of substance abuse (Collins et al., 2009) and self disclosure narratives about recollections of traumatic events or daily events (Moore \& Brody, 2009); however these preliminary findings suggest that there may be a specific linguistic pattern associated with mindful states.

In the Collins et al. (2009) study, linguistic analyses were conducted in participants' responses to open-ended questions related to their impressions of mindfulness practice and the substance use mindfulness-based relapse prevention program. Two word categories of mindfulness language were created. As hypothesized, mindfulness language was correlated with several other linguistic variables of interest. Mindfulness language negatively correlated with impersonal pronouns, past tense, and anger, and positively correlated with words related to affect, body and insight. In addition, the association between mindfulness language and the mindfulness-based relapse prevention program was higher than the association between mindfulness 
language and another treatment manual (i.e., the Big Book). Furthermore, the two mindfulness language categories significantly predicted alcohol and other drugs use days.

Moore and Brody (2009) investigated whether changes in writing predicted mindfulness states. They looked at mindfulness as a unique construct as well as to mindfulness facets (i.e., nonjudgmental acceptance, observing and describing present stimuli, and acting with awareness). Participants wrote about a traumatic event or daily events over three days. Correlations between baseline mindfulness levels and word categories in the first day of writing were conducted. Positive emotion words negatively correlated with nonjudgmental acceptance, and positively correlated with describing present stimuli; self-reference negatively correlated with observing present stimuli. Regarding cognitive processing words, increased use of this word category significantly predicted increased nonjudgmental acceptance, but this was only true for the traumatic condition. Cognitive processing words increases also predicted increases in describing present stimuli and overall mindfulness, but only for women. Changes in verb tenses also predicted changes in mindfulness: increases in present tense were associated with increase in nonjudgmental acceptance, increases in future were associated with observing present stimuli, and increases in past and future tenses significantly predicted describing present stimuli. In addition, present tense partially mediated the relation between nonjudgmental acceptance and the writing conditions (those writing about daily events scored higher in mindfulness).

Although important in advancing our knowledge about the language associated with mindfulness, these studies were conducted with specific samples (i.e., participants with substance abuse and undergraduate students; Collins et al., 2009; Moore \& Brody., 
2009, respectively) which limits the generalizability of these findings. In addition, Collins et al. (2009) did not use a mindfulness measure, but used "mindfulness language" to define it, and Moore and Brody (2009) only used one measure of mindfulness. Thus, more studies are needed in order to validate the association between language categories and mindfulness across different measures.

\section{The Present Study}

Extant research shows that worry presents as a barrier for experiencing what is presented at the present moment. This barrier can be observed in terms of physiological, emotional and cognitive features of worry (Buhr \& Dugas, 2002; Mennin et al., 2002; Thayer et al., 1996). However, despite literature suggesting that worry is primarily verbal (Behar et al., 2005; Borkovec \& Inz, 1990; Freeston et al., 1996; Hirsch et al., 2012), our understanding of the cognitive pattern involved in worry is still limited. In particular, the "internal dialog" characterizing high worriers and worrisome states is still unclear. As previously mentioned, having a better understanding of this internal cognitive activity is essential in better understanding worry and its theoretical models.

Mindfulness presents as a contrasting, healthier state in relation to worry, and has been found to be an effective treatment for worry (Craigie et al., 2008; Kabat-Zinn et al., 1992). This benefit may be due particularly to openness to present moment experiences found in mindful states. That is, mindfulness could serve as an efficient strategy for breaking the experiential barrier found in high worriers. Although limited, we have some understanding of how changes in physiological and emotional aspects associated with worry act as mechanisms of change in mindfulness interventions for worry. However, we currently do not know whether and how linguistic aspects associated with worry change 
in these interventions. Similar to worry, we know little about the internal dialog characteristic of mindfulness, especially in what it relates to aspects of the worry models. Thus, it would not be possible to establish the cognitive changes necessary to transition from a worrisome to a mindful state.

Given the immediate access it provides into cognitions and emotions, linguistics presents as the ideal tool for investigating this internal dialog in worry and also in better understanding mindfulness states as a means of elucidating worry, this contrasting construct (see figure 2). Through these investigations, we could broaden our understanding of theoretical models of worry, and in particular, about how they interact and are integrated, which is an area needing particular attention (Behar et al., 2009). Furthermore, once the internal dialog is also established for mindfulness states, it would be easier to understand the changes that need to occur cognitively to transition between a worrisome and a mindful state. That is, cognitive mechanisms of change could be more easily identified and investigated.

Similar to the findings in the emotional and physiological domains which suggest that worrisome and mindfulness states are antagonist (Feldman et al., 2007; Mennin et al., 2002; Thayer et al., 1996; Vlemincx et al., 2013), it is expected that, indicating their contrasting nature, the same language categories derived from worry and mindfulness theories will predict both worry and mindfulness states, but with opposite relations to the dependent variables. Likewise, worry will present with different proportions of certain language categories, compared to a more neutral cognitive activity (i.e., telling a story). The following hypotheses follow from this conceptualization. 
First, given the avoidant nature of worry (Borkovec, 1994), and the acceptance of present moment experiences characteristic of mindfulness states (Bishop et al., 2004), it was hypothesized that higher worry would be associated with lower use of present tense and higher overall number of words, while higher mindfulness would be associated with higher use of present tense and lower overall number of words.

Likewise, based on the intolerance of uncertainty model of worry (Buhr \& Dugas, 2006) and on the curiosity toward different experiences found in mindfulness states (Bishop et al., 2004), it was hypothesized that higher worry would be associated with higher use of questions, while higher mindfulness would be associated with lower use of questions.

In light of the metacognitive features of worry, which suggest an inflexible and rigid focus on worrisome and anxious metacognitions (Wells, 2006) and of a higherorder, global metacognitive structure proposed in mindful states (Jankowski \& Holas, 2014), it was hypothesized that higher worry would be associated with higher use of anxiety-related words, while higher mindfulness would be associated with lower use of anxiety-related words.

Based on findings associating worry and GAD to emotion dysregulation (Mennin et al., 2005), and on adaptive emotion regulation strategies characteristic of mindfulness (Coffey et al., 2010), it was hypothesized that higher worry would be associated with higher use of negative emotion words, while higher mindfulness would be associated with lower use negative emotion words.

Lastly, based on worry and mindfulness theories (e.g., Behar et al., 2009; Chambers et al., 2008) and linguistic features (e.g., Molina, 1998; Moore \& Brody, 
2009), it was hypothesized that, as compared to the neutral writing task, the worry writing task would elicit lower present tense and higher overall number of words, questions, anxiety related words, and negative emotion words. 


\section{METHODS}

\section{Power Analysis}

Power analyses were conducted in order to determine the necessary sample size. G*Power 3.1.3 (Erdfelder, Faul, \& Buchner, 1996) was used to calculate power for all the analyses. The correlations between worry scores and the language categories required a minimum sample size of 44 participants in order to detect a medium to large effect size $(r=.40)$ with an alpha of .05 and a power of .80 . A multiple linear regression model exploring the effects of the language variables on worry and mindfulness, respectively, required a minimum sample size of 34 participants in order to detect a medium effect size $\left(f^{2}=.25\right)$ with an alpha of .05 and a power of .80 . The multivariate repeated measures ANOVA comparing effects of the five language categories relative to the worry and neutral condition required a minimum sample size of 44 participants in order to detect a medium effect size $\left(\mathrm{f}^{2}=.25\right)$ with an alpha of .05 and a power of .80 . Estimated effect sizes were based on previous studies examining linguistics and psychopathology suggesting medium to large effect sizes (e.g., Geronimi \& Woodruff-Borden, 2015), thus, most power analyses were conservative. Given the power analyses, an a priori recruitment goal of 44 participants was planned.

\section{Participants}

Participants in the current study included 46 English native speaking adult women who ranged in age from 28 to $49(M=39.45, S D=5.46)$. This study was part of a larger 
project about familial anxiety and worry; all participants in this sample were mothers. The majority of the sample had an annual family income of $\$ 60,000$ or above. Participants were highly educated, and the majority (76\%) reported having at least a Bachelor's degree. The majority of participants were European American (89\%), three were African American (7\%), and two (4\%) self described as "other". For additional participant demographics, see Table 1.

Participants were primarily recruited from local schools, after-school programs, mental health agencies, community self-help groups, and other community locations such as sport facilities. Though the study was part of a larger project on familial anxiety and worry, there were no diagnostic requirements for participation in the current study.

\section{Procedure}

Individuals who expressed interest in the study were sent an email with information about the study and a link to review and sign the consent form and complete the questionnaires online through Redcap. Given that the lab tasks and interviews in the larger study required a considerable amount of time (i.e., 2-4 hours), completing the questionnaires prior to the lab visit reduced the burden during the visit and facilitated scheduling. Participants then came to a lab visit for further data collection. The administration of the neutral and worry writing tasks was counterbalanced such that half of the participants completed the neutral task first, and the other half, completed the worry task first.

\section{Measures}

Penn State Worry Questionnaire. (PSWQ; Meyer, Miller, Metzger, \& Borkovec, 1990). The PSWQ is a 16-item self-report inventory designed to measure an 
individual's tendency to worry excessively. Participants indicate how typical a statement is for them using a five-point scale. The measure has been shown to have good internal consistency, test-retest reliability, and convergent validity (Davey, 1993; Molina, Borkovec, Davey, \& Tallis, 1994). The PSWQ internal consistency in the current sample was acceptable $(\alpha=.72)$.

The Five Facet Mindfulness Questionnaire. (FFMQ; Baer, Smith, Hopkins, Krietemeyer, \& Toney, 2006; Baer, Walsh, \& Lykins, 2009) The FFMQ is a self-report questionnaire that measures an individual's propensity to be mindful. Five subscales are included in the questionnaire: nonreactivity to inner experience (nonreactivity), acknowledging thoughts without responding; nonjudging of inner experincing (nonjudging), being nonjudgemental towards thoughts and feelings; observing, the propensity to pay attention to inner experience; describe, the propensity to be able to label inner experiences; and acting with awareness (acting), attending to what arises in the present moment (Baer et al., 2008). The measure has demonstrated excellent psychometric properties (Baer et al., 2009); it has been found to be associated to related constructs, including thought suppression and openness to experience. Cronbach's alphas for the subscales have been in the .82-.91 range in previous investigations (Baer et al., 2006). Internal consistency ranged from acceptable to excellent in the current sample (nonreactivity, $\alpha=.87$; nonjudging, $\alpha=.73$; observing, $\alpha=.84$; describing, $\alpha=.93$, and acting, $\alpha=.91)$. 


\section{Writing Tasks}

Worry task. Participants were asked to transcribe their worries so that their verbal activity during that period could be assessed. The instructions for this task are presented in appendix I.

After giving directions, the experimenter asked the participants if they had questions and left the room. The participants were allowed 10 minutes to transcribe their worries.

Neutral task. Participants were asked to write a brief composition describing something interesting that happened during the last day(s) or week(s). They were given 10 minutes to complete this task. The instructions for this task are in appendix I.

After giving instructions, the experimenter asked if participants had questions and then left the room.

Language analysis. The Linguistic Inquiry and Word Count (LIWC2007; Pennebaker, Chung, Ireland, Gonzalez, \& Booth, 2007) computer software was used for the linguistic analysis. The LIWC2007 uses a hierarchical model to classify words in different word groups (e.g., pronouns, negations). The software generates an output containing the frequency of words in each linguistic category. For the purposes of this study, present tense, overall number of words, number of questions, anxiety-related words and negative emotion words were analyzed. All utterances related to the worry and neutral writing tasks were examined separately through the software, creating a frequency score for the word categories analyzed by each participant by each writing task. The LIWC2007 language categories have been shown to have good internal consistency and 
external validity (Khan, Tobin, Massey, \& Anderson, 2007; Pennebaker et al., 2007;

Tausczik \& Pennebaker, 2010). 


\section{RESULTS}

\section{Preliminary Analyses}

Prior to conducting statistical analyses, all variables were checked for outliers and normal distributions. Outliers were defined, for the purpose of this study, as values beyond three standard deviations above or below the mean. Further, the multivariate analysis of variance (MANOVA) assumptions of multivariate normality, linearity of the dependent variables relationships, and sphericity were verified, as well as well as the regression assumptions of multivariate normality, no multicollinearity, and homoscedasticity. Next, the variables of interest were analyzed in relation to demographic factors through correlations (for age and income) and a one-way ANOVA (for education) in order to determine whether any of the variables significantly related to demographic variables.

Examination of Model Assumptions. Two participants failed to declare their age; given the small percentage (4.34) of those not answering this question and its secondary role in the study, no data imputation was conducted (Field, 2013). One outlier was found for each of the following categories: anxiety-related words for both the worry and neutral conditions, and number of questions. In order to control for possible effects, they were replaced by the value of the mean plus three standard deviations (Field, 2013). Normality of all study variables was assessed using visual inspection of the histograms and skewness examination (with the cut-off of 2.58 due to the small sample size; Field, 
2013). Number of questions in the worry condition and negative emotion words and anxiety related words in both conditions were positively skewed; square root transformations were conducted. All subsequent analyses are based on the transformed values of these variables. Histograms of all study variables are shown in figures 3 to 15 . Even after transformation, although skewness was in the acceptable range for all variables, the graph for anxiety-related words in the neutral condition still suggested skewness. That is, the transformation mostly addressed the skewness related to values different than "zero", however, the square root transformation did not address the large amount of "zeros" in anxiety-related words in the neutral condition, which improved skewness but did not entirely eliminate it. Thus, analyses involving this variable should be interpreted with caution. Other transformation methods (e.g., logarithmic and fraction) could not be performed due to the high number of "zeros" in the data. Analyses involving linearity were performed using the scatterplots of the variables which indicated largely linear, and/or non-curvilinear, relationships between the investigated variables (see figures 15 to 18). Similar to the histogram analyses, the linearity scatterplots revealed a high number of zeros in the anxiety-related words variable for the neutral condition which influenced linearity of this variable in relation to the others.

In examining the MANOVA assumptions, Quantile-quantile (Q-Q) plots demonstrated that the multivariate normality assumption was met (see figures 19-30). Similar to the linearity assumption mentioned above, the assumption of linearity of the dependent variables relationships was examined using the scatterplots of the variables, which indicated largely linear relationships. The sphericity assumption was met for the 
"worry-neutral" condition, but not for language category or the interaction, thus, the Greenhouse-Geiser test was used, as it does not assume sphericity.

Prior to conducting the multiple regressions, assumptions were examined. The multivariate normality assumption was met (see above). Despite the high correlation found between the predictors (i.e., negative emotion words and anxiety related words, $r(46)=.89, p<.001)$, multicollinearity indices still fell within the acceptable limits (VIF $=4.67$; Tolerance $=.21$; O'Brien, 2007). Lastly, the residuals versus predicted values analysis showed that the data were homoscedastic.

Relation Between Study and Demographic Variables. Significant bivariate Pearson correlations emerged between age and worry, $r(46)=-.36, p=.018$; the correlations between age and number of words in the worry condition, $r(46)=-.30, p=$ .051 , and present tense in the neutral condition, $r(46)=-.29, p=.060$, approached significance. No other significant correlations emerged between age and study variables. Income was not associated with any of the study variables. Given the aforementioned significant association between worry and age, age was entered as a covariate in subsequent models involving worry. Further, two One-Way ANOVAs revealed no effect of education on worry and mindfulness, $F s<1$.

Descriptive Statistics. As compared to non-clinical samples $(M=50.74 ; S D=$ 14.79; e.g., Fisak \& von Lehe, 2012), women in the present sample $(M=47.72 ; S D=$ 15.97) endorsed similar levels of worry. The women in the current study endorsed (numerically) higher levels of mindfulness $(M=132.17 ; S D=19.82)$ than expected in comparison to previous community samples. Specifically, psychometric evaluations of the FFMQ in normative and predominately non-Hispanic White community women have 
yielded means around $100.90(S D=14.85)$ for women (e.g., Fisak \& von Lehe, 2012). Means and standard deviations for all study variables can be found in table 2 .

Bivariate Correlations Between Worry and Mindfulness. We explored the association between worry and mindfulness and compared it to previous studies (e.g., Fisak \& von Lehe, 2012). Worry and mindfulness were significantly associated, $r(46)=$ $.55, p<.001$. Regarding mindfulness subscales, although observe and describe did not correlate with worry, acting with awareness $[r(46)=-.30, p=.040]$, nonjudging of inner experience $[r(46)=-.51, p<.001]$, and non reactivity to inner experience $[r(46)=-.61, p$ $<.001]$ were significantly negatively associated with worry. Table 3 displays the bivariate Pearson correlations between worry and mindfulness, as well as each subscale of mindfulness as measured by the FFMQ.

\section{Hypotheses Testing}

\section{Hypothesis 1.}

Higher worry will be associated with lower use of present tense and higher overall number of words, while higher mindfulness will be associated with higher use of present tense and lower overall number of words.

Two-tailed bivariate Pearson correlations were conducted between worry and mindfulness scores, respectively, and present tense for each writing condition. No significant associations emerged from these analyses [worry, worry condition $r(46)=$ $.08, p=.602$; neutral condition, $r(46)=.18, p=.233$; mindfulness, worry condition $r(46)$ $=.09, p=.538$; neutral condition, $r(46)=-.10, p=.510$ ] and number of words [worry, worry condition $r(46)=.23, p=.130$; neutral condition, $r(46)=.04, p=.784$; 
mindfulness, worry condition $r(46)=-.09, p=.547$; neutral condition, $r(46)=-.04, p=$ $.790]$.

\section{Hypothesis 2.}

Higher worry will be associated with higher use of questions, while higher mindfulness will be associated with lower use of questions.

Two-tailed bivariate Pearson correlations revealed no association between worry and use of questions for either writing condition [worry condition $r(46)=.12, p=.434$; neutral condition, $r(46)=.24, p=.100]$. Similarly, no association was found between mindfulness scores and use of questions in either writing condition [worry condition $r(46)=.20, p=.174$; neutral condition, $r(46)=-.25, p=.09]$.

\section{Hypothesis 3.}

Higher worry will be associated with higher use of anxiety-related words, while higher mindfulness will be associated with lower use of anxiety-related words.

Two-tailed bivariate Pearson correlations were conducted between worry and mindfulness scores, respectively, and anxiety-related words for each writing condition. Worry was significantly correlated with anxiety-related words in the worry condition, $r(46)=-.44, p=.002$, such that those with higher worry tended to use less anxiety related words. No association was found, however, in the neutral condition, $r(46)=.08, p=.581$. Further, while no significant relationship was established between mindfulness and anxiety-related words in either condition [worry: $r(46)=.11, p=.448$; neutral: $r(46)=$ $.06, p=.701]$, a marginally significant association was found between the nonreactivity scale of the FFMQ and anxiety related words, $r(46)=.28, p=.059$. This pattern was not found for the neutral condition, $r(46)=.01, p=.988$. A similar finding emerged for the 
nonjudgement subscale, such that a significant association was found between the nonjudgement scale of the FFMQ and anxiety-related words, $r(46)=.33, p=.024$, but no association was found in the neutral condition, $r(46)=.01, p=.966$. These correlations revealed that those who scored higher in worry tended to use less anxiety-related words, while those higher in mindfulness, nonreactivity and nonjudgement in particular, tended to use more anxiety-related words, which contrasts with the study predictions.

\section{Hypothesis 4.}

Higher worry will be associated with higher use of negative emotion words, while higher mindfulness will be associated with lower use negative emotion words.

Two-tailed bivariate Pearson correlations were conducted between worry and mindfulness scores, respectively, and negative emotion words for each writing condition. Similar to the findings related to the hypothesis 3 , a significant association emerged between worry and negative emotion words, $r(46)=-.36, p=.014$, and nonreactivity and negative emotion words, $r(46)=.32, p=.028$, but only in the worry condition [neutral condition, worry: $r(46)=.11, p=.481$; nonreactivity: $r(46)=-.06, p=.701]$. Further, a slight trend towards significance was found between the nonjudgement scale of the FFMQ and negative emotion words in the worry condition, $r(46)=.25, p=.090$. No significant pattern emerged in the neutral condition, $r(46)=-.07, p=.642$. The correlations revealed that those who scored higher in worry and lower in nonreactivity tended to use less negative emotion words, while those higher in mindfulness and lower in worry tended to use more negative emotion words, which contrasts with the study predictions. 


\section{Hypothesis 5.}

As compared to the neutral writing task, the worry writing task will elicit lower present tense and higher overall number of words, questions, anxiety-related words, and negative emotion words.

A multivariate repeated measures analysis of variance, MANCOVA, was conducted comparing language use for all language categories during the worry and neutral writing tasks. Two factors, language category (i.e., present tense, number of words, anxiety-related words, number of questions, negative emotion words) and condition (worry and neutral) were entered in the MANCOVA in order to make it possible to test for each word category in each condition, and age was entered as a covariate. For the purpose of this study, only the condition effect was relevant. The condition effect was significant, $F(1,42)=7.52 ; p=.009 ;$ partial $\eta^{2}=.15$ (interaction term, language vs. condition vs. parent age, $F(4,42)=7.41 ; p=.009 ;$ partial $\left.\eta^{2}=.15\right)$.

Follow up univariate mean comparisons for each condition (worry and neutral activity), were conducted using paired-samples t-tests to determine which of the language categories separately showed a condition effect. As five comparisons were conducted, alpha level was adjusted to .01, according to the Bonferroni correction. There was no significant effect of condition for number of words, $t(45)=.76, p=.448$, and number of questions, $t(45)=.19, p=.851$. However, there were significant differences for anxietyrelated words, $t(45)=6.80, p<.001$, present tense, $t(45)=10.63, p<.001$, and negative emotion words, $t(45)=6.91, p<.001$. The worry condition scores were higher for these three language categories (means and standard deviations for language variables in each 
category can be found in Table 2). With the exception of present tense, the mean differences fell in the expected direction.

\section{Exploratory Analyses}

Following correlational analyses, exploratory regressions will be conducted predicting worry and mindfulness, separately, from the word categories showing significant correlations for each condition, as a way to quantify the amount of variance accounted for in both worry and mindfulness by language categories, as well as to preliminarily test whether certain word categories uniquely predict worry or mindfulness, or whether most of the variance is shared between them in predicting worry and mindfulness, respectively.

Given the association between worry and age, age was entered as a covariate at step 1 of the regression model predicting worry. As language categories were correlated with the nonreactivity and nonjudgement subscales of the FFMQ, but not with mindfulness, these subscales were used in the following analyses. As the significant findings occurred only for the worry condition, only this condition was analyzed in the regressions below.

Worry Regression. Block 1 was significant and age predicted worry scores, $F(1$, $42)=6.08, p=.018 ; R^{2}=.13$. Block 2 was also significant $\left[F(3,40)=5.34, p=.003 ; R^{2}\right.$ $=.29$ ], and language explained an additional $15.95 \%$ of the variance in worry. Analyses of the contribution of each category separately revealed that negative emotion words $(\beta=$ $.06 ; p=.817)$ and anxiety-related words $(\beta=-.46 ; p=.090)$ were not unique predictors of worry, suggesting that most of the variance is shared between these two variables in predicting worry. See regression model in table 4. 
Nonreactivity Regression. The two language categories combined did show a slight trend toward significance in predicting nonreactivity scores, $F(2,43)=2.51, p=.093$, and language explained $10.46 \%$ of the variance in nonreactivity. Analyses of the contribution of each category separately revealed that negative emotion words $(\beta=.32 ; p$ $=.271)$ and anxiety-related words $(\beta=.01 ; p=.978)$ were not unique predictors of nonreactivity, suggesting that most of the variance is shared between these two variables in predicting nonreactivity. See regression model in table 5.

Nonjudgement Regression. Negative emotion and anxiety words combined approached significance in predicting nonjudgement scores, $F(2,43)=2.77, p=.074$, and language explained $11.43 \%$ of the variance in nonjudgement. Analyses of the contribution of each category separately revealed that negative emotion words $(\beta=-.13$; $p=.651)$ and anxiety-related words $(\beta=.44 ; p=.125)$ were not unique predictors of nonjudgement, suggesting that most of the variance is shared between these two variables in predicting nonreactivity. See regression model in table 6 .

\section{Additional Exploratory Analysis}

Predicting Worry from Mindfulness and Language. Given the established association between worry and mindfulness (Fisak \& von Lehe, 2012) and the elaboration of linguistic hypotheses based on worry and mindfulness theoretical models (Behar et al., 2009; Jankowski \& Holas, 2014), a regression was conducted in order to examine whether language accounted for additional variance in worry, when controlling for mindfulness. Given the association between worry and age, this variable was entered in the first block. Mindfulness was entered in the second block and language categories (i.e., negative emotion and anxiety) were entered in the third block. Age significantly 
predicted worry scores, $F(1,42)=6.08, \beta=-.36, p=.018$. Mindfulness also significantly predicted worry, $F(2,41)=17.35, \beta=-.58, p<.001$, and explained an additional $33.18 \%$ of the variance in the model. Language significantly predicted worry as well, $F(4,39)=12.40, p<.001$, and language categories accounted for an additional $10.14 \%$ of the variance in worry scores. Analyses of the contribution of each predictor separately at block three revealed that the language categories were not unique predictors, suggesting that they share variance in predicting worry (anxiety-related words: $\beta=-.19$; $p=.385$; negative emotion words: $\beta=-.14 ; p=.494$ ). 


\section{DISCUSSION}

The present study sought to examine the linguistic features associated with worry and mindfulness. In particular, the study aimed to investigate whether language features derived from worry theoretical models would be associated with worry and mindfulness. To achieve this goal, the study examined several specific language categories (i.e., number of words, present tense, questions, anxiety-related words, and negative emotion words) in relation to worry and mindfulness traits as well as to worry and a neutral writing activity. Several aspects of the proposed association between mindfulness and worry and linguistic features were tested. Specifically, it was predicted that present tense would be negatively correlated with worry and positively correlated with mindfulness and that overall number of words, number of questions, anxiety-related words, and negative emotion words would be positively correlated with worry and negatively correlated with mindfulness. Further, it was hypothesized that present tense use would be lower during worry than during neutral writing, while overall number of words, number of questions, anxiety-related words, and negative emotion words would be higher during worry than during neutral writing.

\section{Preliminary Analyses}

Preliminary analyses revealed that, consistent with previous findings, worry and mindfulness were significantly correlated (e.g., Fisak \& von Lehe, 2012). Correlations between the specific scales of the FFMQ and worry were also conducted. While observe 
and describe did not correlate with worry, acting with awareness, nonjudging of inner experience, and nonreactivity to inner experience were significantly negatively associated with worry. This finding is consistent with previous studies and suggests that worry is related to the more complex aspects of mindfulness which require elaborated cognitive activity (Baer et al., 2006). Mindfulness has been conceptualized as a multifaceted construct (Baer et al., 2006). Observe and describe are elementary facets of mindfulness through which the individual notices and labels internal experiences (Baer et al., 2008). Nonreactivity, nonjudging, and acting with awareness relate to the way in which one responds to internal stimuli, and these very responses may be the ones debilitated in worry given its avoidant and emotionally dysregulated nature (Fisak \& von Lehe, 2012; Behar et al., 2009). These results are also consistent with Fisak and von Lehe's (2012) findings and their interpretation that one's reactions to worry may be more closely associated with worry-related symptoms than one's propensity to notice and describe the worry process.

\section{Hypotheses Testing}

The hypothesis that present tense would be negatively associated with worry and positively associated with mindfulness while overall number of words would be positively associated with worry and negatively associated with mindfulness was not supported. This finding diverges from previous research investigating worry and individuals with GAD which suggests a significant role of temporal orientation, particularly present tense, in those who worry excessively (Geronimi \& WoodruffBorden, 2015). In the study conducted by Geronimi and Woodruff-Borden (2015), present tense significantly classified those with GAD, such that those higher in worry 
used less present tense. Our findings of no associations between mindfulness facets and present tense also contrast with Moore and Brody's (2009) study in which increases in present tense words predicted increases in the nondjudgemental acceptance feature of mindfulness, and this finding applied to both traumatic and daily event themes. Their findings suggest that those who focus more on the present moment experience are better at being nonjudgemental towards their reality.

There are a few factors that could have influenced the differences in findings. Our two writing tasks were thematically different than theirs (i.e., transcribing worries and writing about something interesting that happened versus writing about traumatic experiences and daily events). The questionnaires assessing mindfulness were distinct (FFMQ versus and The Kentucky Inventory of Mindfulness Skills, KIMS; Baer, Smith, \& Allen, 2004) and, thus, the studies were assessing different dimensions of the construct. While three scales of these measures share similar underlying features (i.e., observing, describing, and acting with awareness), the nonjudgement scale of the KIMS (i.e., accept without judgment) has a higher emphasis on acceptance and there is no equivalent for nonreactivity in that measure. It is possible that present tense is more strongly associated with the subscale accept without judgement of the KIMS used in Moore and Brody's (2009) study than the FFMQ nonjudgement scale because it relates to aspects regarding accepting one's present reality. Further, in their study, narratives were collected over three days, and their sample was younger $\left(M_{\text {age }}=18.88\right)$ and included males. The prompts in our study may also influence the discrepancy. That is, the neutral prompt asked them to think of an event in the past and the worry prompt, although discussing a past worry, still seems to invoke a worry that is presently occurring as well. 
Given the contrasting findings and differences in methodology, future research is needed in order to establish the nature of the association between worry and mindfulness and temporal orientation.

The hypothesis that worry would be positively correlated with number of questions and mindfulness would be negatively correlated with number of questions was not supported. This finding contrasts with the IU model which posits uncertainties are not well tolerated by higher worriers (Buhr \& Dugas, 2006) and, as a consequence, those higher in worry would face more questions. This finding is also discrepant from previous research that found an association between GAD and language (Geronimi \& WoodruffBorden, 2015). However, again, there are considerable differences in the method used by these studies that could account for the discrepancy in the results. For instance, in Geronimi and Woodruff-Borden's (2015) study, language samples were extracted from a free play interaction between mother and child such that language was expressed in a verbal format. No studies have been conducted in regards to the association between mindfulness and the use of questions. Similar to the previous hypothesis, future research is needed in order to establish the nature of the association between mindfulness and use of questions.

The hypothesis that worry would be positively correlated with anxiety-related words and mindfulness would be negatively correlated with anxiety-related words was not supported. However, significant findings emerged. First, worry was negatively correlated with anxiety-related words in the worry condition. Our original hypothesis was based on the idea that, as a consequence of experiencing higher anxiety (Muris, Roelofs, Meesters, \& Boomsma, 2004), those higher in worry would express more anxiety-related 
words in their worry transcripts. On the other hand, it is possible that those higher in worry are using less anxiety-related words as an attempt to avoid experiencing or labeling intense emotions (Peasley-Miklus \& Vrana, 2000). Originally, this hypothesis was based on the metacognitive features of worry and the understanding that worry is defined by an inflexible and rigid cognitive activity/style (Wells, 2006). The findings, however, suggest that lower use of anxiety-related words could be an attempt to avoid emotional content, which would be consistent with the avoidance and emotion regulation worry models. In particular, these models conceptualize worry as a strategy to avoid, control, and minimize emotional experiences (Menning et al., 2002; Peasley-Miklus \& Vrana, 2000). Expressing lower emotional content in regards to the worry writing task could be a way of accomplishing this goal.

It is important to emphasize that emotional content interpretations should be viewed very cautiously as our method consisted of word count. More precisely, our variables are derived from percentages of a word category over the overall number of words. While allowing the identification of linguistic patterns, this method is limited as it does not take semantics or more qualitative analyses into account. There was also a significant positive association between anxiety-related words and nonjudgement as well as a marginally significant association between anxiety related words and nonreactivity, both in the worry condition. These two mindfulness facets have previously shown the strongest associations with worry (e.g., Fisak \& von Lehe, 2012); thus, it is not surprising that they are the ones with closer associations with anxiety-related words. In particular, nonjudging, or taking a nonjudgemental attitude towards thoughts and emotions, and nonreactivity, defined as noticing thoughts without reacting to them (Baer et al., 2006, 
2009), are characterized by welcoming any emotional state, including a negative one (Baer et al., 2009).

It was hypothesized that worry would be positively correlated with negative emotion words and mindfulness would be negatively associated with negative emotion words. Associations were found between worry and mindfulness and this word category; however, these associations were not in the expected direction. More specifically, in the worry condition, worry and negative emotion words were negatively associated. This hypothesis was primarily based in the emotion dysregulation worry model which suggests that high worriers experience more intense emotions (Mennin et al., 2002; Turk et al., 2005). Nonetheless, it is possible that as suggested by the avoidance worry model (Behar, 2009), worrying is an attempt to avoid experiencing negative emotions and not verbalizing them may be one way of accomplishing this goal (Borkovec, 1994). It is important to mention that, as with the other language variables, the emotion words variable in this study consisted only of the percentage of emotion words in the transcripts based on the simple sum of this word category. Thus, any interpretations should be taken cautiously and the specific context in which emotions were mentioned was not analyzed. Future studies focusing on more qualitative methods should address this important aspect of the worry process. Also, contrary to expectations, negative emotion words were positively correlated with nonreactivity and the association with nonjudging revealed a trend towards significance; these findings were true only for the worry condition. Acceptance towards one's experiences, characteristic of these mindfulness subscales (Baer, 2006, 2009), might partially explain these findings and suggest that those higher in 
this trait may be more comfortable with having cognitive processes characterized by negative emotions naturally unfold (Baer, 2003).

Subsequent exploratory regression analyses also revealed that, after controlling for age, anxiety-related words and negative emotion words contributed significant variance to worry, although no language variable emerged as a unique predictor. This finding might be explained by the fact that these two language variables were also correlated and share several commonalities. Further, negative emotion words and anxiety related words accounted for a substantial portion of the variance in nonreactivity (10.46\%) and nonjudgement (11.46), however, this model failed to reach significance.

The hypothesis that worry activity would differ from a neutral writing activity on the proposed language categories was partially supported. First, an overall multivariate effect was found for worry versus neutral condition. No effect was detected for number of words and questions; however, worry activity was associated with more present tense, anxiety-related words, and negative emotion words than the neutral writing activity. Although the results related to present tense diverge from those found in Molina et al.'s (1998) study, the findings regarding anxiety and emotion words are consistent with their study. Molina et al. (1998) found that the worry period had lower present tense use than a neutral period. Their study is methodologically considerably different from the present study, and this could in part account for these discrepancies. The fact that our neutral task consists of writing about an interesting event that happened could have affected temporal orientation by the use of more past tense and less present tense in the neutral task, as mentioned above. In Molina et al. (1998) study, the neutral task was to "simply thinkaloud." The present study did, however, concord with Molina et al.'s (1998) findings 
regarding emotion words as they found that high negative affect statements were higher during worry. As opposed to temporal orientation, which may depend on the cognitive activity worry is being contrasted with, affect may be a core constituent that is consistently salient when worry is contrasted to different cognitive activities. Future studies should continue to test affect expression through language in order to validate these findings.

Overall, results provide support for the notion that language expression is relevant in understanding worry and mindfulness as traits, similar to previous studies exploring psychopathology features and mindfulness as they relate to language expression (e.g., Hofmann et al., 2012; Moore \& Brody, 2009). The study findings suggest that linguistic elements mainly derived from the emotion dysregulation and avoidance worry models (Behar et al., 2009) are associated with worry and mindfulness. Interestingly, those higher in mindfulness and lower in worry used more negative emotion words and anxiety-related words in their worry, a reverse pattern in relation to that found in the present study when comparing worry to neutral writing activity. That is, worry as a cognitive process had more negative emotion words and anxiety-related words, as expected. It appears that those higher in worry and lower in mindfulness use cognitive elements associated with worry activity (e.g., negative emotion words, anxiety-related words) less often than other individuals, possibly as an attempt to avoid eliciting negative affect (e.g., Behar et al., 2009). In contrast, these findings suggest that those higher in mindfulness, especially nonreactivity and nonjudgement, and lower in worry appear to allow those emotions to occur during the worry process, consistent with mindfulness conceptualizations and established associations with worry (Fisak \& von Lehe, 2012). 


\section{Additional Exploratory Analyses}

A regression was conducted in order to examine whether language accounted for additional variance in worry when mindfulness was also considered. Language categories accounted for a significant portion of the variance in worry scores beyond that explained by mindfulness. Anxiety related words and negative emotion words did not, however, emerge as unique predictors of worry, which suggest the variance explained by language is shared by these categories. Although the proposed language categories associated with worry and mindfulness were based on conceptual features of these constructs (e.g., Behar, 2009; Fisak \& von Lehe, 2012), through this model, it appears that there may be a unique quality to language expression that complements the association between worry and mindfulness.

\section{Implications of Linguistic Features for Worry and Mindfulness}

\section{Conceptualizations and Treatment Development}

The results of the current study have important implications on conceptualizations of worry and mindfulness. Results suggest that language use is associated with worry and mindfulness. Specifically, lower levels of anxiety-related words and negative emotion words likely are related to higher levels of worry and lower levels of mindfulness traits in female adults who were mothers. Further, worry writing activity, as compared to neutral writing activity, shows higher use of present tense, anxiety related words, and negative emotion words. Although additional research is necessary to solidify these findings, the current study suggests that linguistic elements are important in understanding worry and mindfulness. Interestingly, the pattern of language categories (negative emotion words and anxiety-related words, in particular) is reversed. That is, while those higher in worry 
and lower in mindfulness used less negative emotion words and anxiety-related words in their worry, the worry writing activity was characterized by higher use of these word categories as compared to a neutral writing cognitive activity.

As mentioned above, this reverse linguistic pattern may indicate avoidance of emotions for individuals high in worry/low in mindfulness, and, in contrast, nonreactivity or nonjudgement towards emotions for individuals low in worry/high in mindfulness (Fisak \& von Lehe, 2012). Counterintuitively, the worry of those lower in worry/higher in mindfulness, appears to have, linguistically, more elements that constitute the worry activity (e.g., anxiety related words; negative emotion words). Future studies should continue to investigate linguistic patterns in order to replicate and provide additional support to the present findings and more fully explore language as a way to further understand worry and mindfulness, both as traits and as states.

This research could be important in determining clinical applications of psycholinguistics. Recent evidence suggests that there may be relevant linguistic markers in the treatment of GAD (Dirkse \& Hadjistavropoulos, 2015). Dirkse and Hadjistavropoulos (2015) found that over the course of internet delivered CBT, the use of negative emotion, anxiety, causation, and insight words decreased during therapy sessions, whereas past tense use increased. It would also be interesting to analyze changes in worry activity language during treatment in order to determine linguistic changes in worry as a result of CBT. While Dirkse and Hadjistavropoulos's (2015) study suggests that the use of negative emotion words decreased during the course of treatment, it could be found that this same word category increases in worry activity during the course of treatment as individuals become less avoidant of their own emotions, similar to 
the present study findings regarding the worry of those higher in mindfulness and lower in worry. As such, once the language associated with worry and GAD is established, language analysis could be a complementary instrument in the treatment and assessment of worry and GAD.

\section{Limitations and Future Research}

The current findings are restricted by several limitations. First, worry is a verbal, internal mental process (Hirsch et al., 2012) and the simple task of transcribing its content could have implications on its ecological validity. That is, transcribing it could lead to an involuntary search for terms that would likely not happen when one is just worrying and not asked to do it as part of a research project. Similarly, typing could elicit the thought, similar to school and work assignments, that there is a correct way of doing it, or an appropriate way to worry, undermining the validity of the content. Previous studies investigating the language associated with worry or with individuals meeting criteria for GAD assessed verbal spoken language (Molina et al., 1998; Geronimi \& WoodruffBorden, 2015). These studies recognized the possible concerns associated with this method, such as the transformation of an internal cognition into purely verbal content. However, it is also possible that written language is not the most effective way of assessing worry due to the potential drawbacks described above. Further, possibly impacting ecological validity is the fact that participants were asked to "worry" during a brief period of time whereas worry is usually elicited by internal stimuli (Tallis \& Eysenck, 1994). Analyzing worry during a limited period of time could have affected the ability to capture certain language patterns that are expressed in longer periods.

Desirability effects in relation to the interviewer or researcher could also interfere in the 
worry process itself making it less or more intense, for instance. Future studies should use methods that assess worry activity closer to its natural manifestation. For instance, worry could be recorded, either through speech or written format, in the individual's environment during several days. Preliminary findings from an earlier study indicate that worry was correlated with language variables (e.g., negations and future) collected naturalistically across seven days in a small sample of adolescents (Geronimi et al., 2015). Similar investigations with larger samples and across the life span are warranted to better understand worry and mindfulness language over time.

In addition to ecological validity concerns, characteristics of the current sample could have affected the results. The current sample was composed exclusively by women, mothers, in a limited age range, predominantly European American, and highly educated. While worry is more prevalent in women (Robichoud, Dugas, \& Conway, 2003), and there is a need for better understanding the phenomenon in this population, limiting the sample to only women does not allow for gender comparisons. In addition, despite the fact that women in this sample endorsed similar levels of worry as compared to nonclinical samples, they presented with higher levels of mindfulness compared to previous findings investigating community samples (Fisak \& von Lehe, 2012). Generalizations of the current findings to other female populations should be made carefully given that they represent a specific subset of this population. Future research should investigate the language of mindfulness and worry in a more diverse population. That is, investigations should include gender diversity, a wider age range, different educational backgrounds, a range of income levels, and more diverse ethnicities. 
The present study focused exclusively on individuals who had English as their native language. The language one speaks influences cognitions (Silva, 2014). Thus, investigating worry and mindfulness language cross-culturally might provide important information about linguistic elements associated with worry and mindfulness in different languages and potentially inform culturally sensitive treatments.

Finally, as mentioned previously, the quantitative method used to analyze the transcripts (i.e., word count) should be interpreted very cautiously. Qualitative methods such as semantic/thematic analyzes could address this limitation by the use of coding methods targeting specific elements of worry models and mindfulness. In addition, some language categories used in this and other studies should also be analyzed taking semantics into account and these may capture non-grammatically structured questions, for instance. Semantic analyses allow for broader operationalization of word/sentence categories which could help detecting speculative language or questions in speech. Although the frequency of certain word categories may suggest important linguistic patterns, the use of qualitative research will be necessary in further elucidating the features associated with worry and mindfulness linguistic features.

\section{Conclusion}

This is the first study using linguistics to investigate features of worry models associated with worry and mindfulness concomitantly. Results of the present investigation suggest that word categories related to emotions (i.e., anxiety and negative emotion words) hold associations with worry and mindfulness. Number of words, questions, and present tense did not reveal an association with worry and mindfulness levels in the present sample. Further, compared to a neutral task, worry activity had 
higher use of present tense, anxiety-related words, and negative emotion words. These findings suggest that features of the Avoidance and Emotion Disregulation worry models (Behar et al., 2009) are linguistically represented in worry through the use of less negative emotion and anxiety-related words by those higher in worry, and a reverse pattern occurred for those higher in mindfulness. This finding may reflect avoidance of emotional content in those higher in worry and lower in mindfulness and acceptance of emotional experience by those lower in worry and higher in mindfulness. Future research should continue exploring linguistic features of worry and mindfulness in order to establish the relevant linguistic variables associated with these constructs, and, concomitantly, explore their clinical applications in evidence-based treatments for worry and assessment. 


\section{REFERENCES}

American Psychiatric Association. (2013). Diagnostic and statistical manual of mental disorders (5th ed.). Washington, DC: American Psychiatric Publishing.

Arch, J. J., \& Craske, M. G. (2006). Mechanisms of nonreactivity: Emotion regulation following a focused breathing induction. Behaviour Research and Therapy, 44(12), 1849-1858. doi: 10.1016/j.brat.2005.12.007

Baer, R. A. (2003). Nonreactivity training as a clinical intervention: A conceptual and empirical review. Clinical Psychology: Science and Practice, 10(2), 125-143. doi: 10.1093/clipsy/bpg015

Baer, R. A., Smith, G. T., \& Allen, K. B. (2004). Assessment of mindfulness by selfreport: The Kentucky Inventory of Mindfulness Skills. Assessment, 11(3), 191-206.

Baer, R. A., Smith, G. T., Hopkins, J., Krietemeyer, J., \& Toney, L. (2006). Using selfreport assessment methods to explore facets of mindfulness. Assessment, 13(1), 27 45.

Baer, R. A., Smith, G. T., Lykins, E., Button, D., Krietemeyer, J., Sauer, S., ... \& Williams, J. M. G. (2008). Construct validity of the five facet mindfulness questionnaire in meditating and nonmeditating samples. Assessment, 15(3), 329-342.

Baer, R. A., Walsh, E., \& Lykins, E. L. (2009). Assessment of mindfulness. In Clinical handbook of mindfulness (pp. 153-168). Springer, New York, NY. 
Behar, E., DiMarco, I. D., Hekler, E. B., Mohlman, J., \& Staples, A. M. (2009). Current theoretical models of generalized anxiety disorder (GAD): Conceptual review and treatment implications. Journal of Anxiety Disorders, 23(8), 1011-1023. doi: 10.1016/j.janxdis.2009.07.006

Behar, E., Zuellig, A. R., \& Borkovec, T. D. (2005). Thought and imaginal activity during worry and trauma recall. Behavior Therapy, 36(2), 157-168. doi: $10.1016 / \mathrm{S} 0005-7894(05) 80064-4$

Bishop, S. R., Lau, M., Shapiro, S., Carlson, L., Anderson, N. D., Carmody, J., ... Devins, G. (2004). Nonreactivity: A proposed operational definition. Clinical Psychology: Science and Practice, 11(3), 230-241. doi: 10.1093/clipsy/bph077

Borkovec, T. D. (1994). The nature, functions, and origins of worry. In Worrying: Perspectives on theory, assessment and treatment. (pp. 5-33). Retrieved from http://www.redibw.de/db/ebsco.php/search.ebscohost.com/login.aspx?direct=true \&db=psyh\&AN=1 994-97842-001\&site=ehost-live

Borkovec, T. D., \& Inz, J. (1990). The nature of worry in generalized anxiety disorder: A predominance of thought activity. Behaviour Research and Therapy, 28, 153-158. doi: 10.1016/0005-7967(90)90027-G

Borkovec, T. D., \& Roemer, L. (1995). Perceived functions of worry among generalized anxiety disorder subjects: Distraction from more emotionally distressing topics? Journal of Behavior Therapy and Experimental Psychiatry, 26(1), 25-30. doi: 10.1016/0005-7916(94)00064-S 
Brockman, R. N. (2017). Emotion Regulation Strategies in Daily Life: Examining Contextual Variability in the Process of Emotion Regulation.

Brockman, R., Ciarrochi, J., Parker, P., \& Kashdan, T. (2017). Emotion regulation strategies in daily life: mindfulness, cognitive reappraisal and emotion suppression. Cognitive Behaviour Therapy, 46(2), 91-113.

Buhr, K., \& Dugas, M. J. (2002). The intolerance for ambiguity scale: Psychometric properties of the English version. Behaviour Research and Therapy, 40, 931-945.

Buhr, K., \& Dugas, M. J. (2006). Investigating the construct validity of intolerance of uncertainty and its unique relationship with worry. Journal of Anxiety Disorders, 20(2), 222-236. doi: 10.1016/j.janxdis.2004.12.004

Burg, J. M., Wolf, O. T., \& Michalak, J. (2012). Mindfulness as self-regulated attention: Associations with heart rate variability. Swiss Journal of Psychology, 71(3), 135139. doi: 10.1024/1421-0185/a000080

Cartwright-Hatton, S., \& Wells, A. (1997). Beliefs about worry and intrusions: The MetaCognitions Questionnaire and its correlates. Journal of Anxiety Disorders, 11(3), 279-296. doi: 10.1016/S0887-6185(97)00011-X

Chambers, R., Lo, B. C. Y., \& Allen, N. B. (2008). The impact of intensive nonreactivity training on attentional control, cognitive style, and affect. Cognitive Therapy and Research, 32(3), 303-322. doi: 10.1007/s10608-007-9119-0

Coffman, S. G. (2013). Cognitive behavioral therapy's nonreactivity concepts reflect both Buddhist traditions and Native American medicine. The Behavior Therapist, 36(6), $156-157$. 
Coffey, K. A., Hartman, M., \& Fredrickson, B. L. (2010). Deconstructing Nonreactivity and Constructing Mental Health: Understanding Nonreactivity and its Mechanisms of Action. Nonreactivity, 1(4), 235-253. doi: 10.1007/s12671-010-0033-2

Collins, S. E., Chawla, N., Hsu, S. H., Grow, J., Otto, J. M., \& Marlatt, G. A. (2009). Language-based measures of nonreactivity: initial validity and clinical utility. Psychology of Addictive Behaviors, 23(4), 743.

Craigie, M. A., Rees, C. S., Marsh, A., \& Nathan, P. (2008). Nonreactivity-based Cognitive Therapy for Generalized Anxiety Disorder: A Preliminary Evaluation. Behavioural and Cognitive Psychotherapy, 36(05), 553. doi: $10.1017 / \mathrm{S} 135246580800458 \mathrm{X}$

Crouch, T. A., Lewis, J. A., Erickson, T. M., \& Newman, M. G. (2017). Prospective investigation of the contrast avoidance model of generalized anxiety and worry. Behavior therapy, 48(4), 544-556.

Davey, G. C. (1993). A comparison of three worry questionnaires. Behaviour Research and Therapy, 31(1), 51-56.

Davidson, R. J. (2010). Empirical explorations of nonreactivity: conceptual and methodological conundrums. Emotion, 10(1), 8-11. doi: 10.1037/a0018480

Davis, R. N., \& Valentiner, D. P. (2000). Does meta-cognitive theory enhance our understanding of pathological worry and anxiety? Personality and Individual Differences, 29(3), 513-526. doi: 10.1016/S0191-8869(99)00211-1

Decker, M. L., Turk, C. L., Hess, B., \& Murray, C. E. (2008). Emotion regulation among individuals classified with and without generalized anxiety disorder. Journal of Anxiety Disorders, 22, 485-494. doi: 10.1016/j.janxdis.2007.04.002 
Delgado, L. C., Guerra, P., Perakakis, P., Vera, M. N., del Paso, G. R., \& Vila, J. (2010). Treating chronic worry: Psychological and physiological effects of a training programme based on nonreactivity. Behaviour Research and Therapy, 48(9), 873882. doi: $10.1016 /$ j.brat.2010.05.012

Delgado-Pastor, L. C., Ciria, L. F., Blanca, B., Mata, J. L., Vera, M. N., \& Vila, J. (2015). Dissociation Between the Cognitive and Interoceptive Components of Nonreactivity in the Treatment of Chronic Worry. Journal of Behavior Therapy and Experimental Psychiatry, 48, 192-199. doi: 10.1016/j.jbtep.2015.04.001

Dirkse, D., Hadjistavropoulos, H. D., Hesser, H., \& Barak, A. (2015). Linguistic analysis of communication in therapist-assisted internet-delivered cognitive behavior therapy for generalized anxiety disorder. Cognitive behaviour therapy, 44(1), 21-32.

Dugas, M. J., Gagnon, F., Ladouceur, R., \& Freeston, M. H. (1998). Generalized anxiety disorder: a preliminary test of a conceptual model. Behaviour Research and Therapy, 36(2), 215-226. doi: 10.1016/S0005-7967(97)00070-3

Dugas, M. J., Gosselin, P., \& Ladouceur, R. (2001). Intolerance of uncertainty and worry: Investigating specificity in a nonclinical sample. Cognitive Therapy and Research, 25(5), 551-558. doi: 10.1023/A:1005553414688

Erdfelder, E., Faul, F., \& Buchner, A. (1996). GPOWER: A general power analysis program. Behavior Research Methods, Instruments, \& Computers,28(1), 1-11.

Erisman, S. M., \& Roemer, L. (2010). A preliminary investigation of the effects of experimentally induced nonreactivity on emotional responding to film clips. Emotion, 10(1), 72-82. doi: 10.1037/a0017162 
Evans, S., Ferrando, S., Findler, M., Stowell, C., Smart, C., \& Haglin, D. (2008). Nonreactivity-based cognitive therapy for generalized anxiety disorder. Journal of Anxiety Disorders, 22(4), 716-721. doi: 10.1016/j.janxdis.2007.07.005

Farb, N. A. S., Anderson, A. K., Mayberg, H., Bean, J., McKeon, D., \& Segal, Z. V. (2010). “Minding one's emotions: Nonreactivity training alters the neural expression of sadness": Correction to Farb et al (2010). Emotion, 10(2), 215. doi: $10.1037 / \mathrm{a} 0019263$

Feldman, G., Hayes, A., Kumar, S., Greeson, J., \& Laurenceau, J. P. (2007). Nonreactivity and emotion regulation: The development and initial validation of the Cognitive and Affective Nonreactivity Scale-Revised (CAMS-R). Journal of Psychopathology and Behavioral Assessment, 29(3), 177-190. doi: 10.1007/s10862$006-9035-8$

Field, A. (2013). Discovering Statistics Using IBM SPSS Statistics: And Sex and Drugs and Rock "N" Roll (4th ed.) London, UK: Sage Publications Ltd.

Field, J. (2005). Language and the Mind. New York: Routledge.

Fisak, B., \& von Lehe, A. C. (2012). The relation between the five facets of mindfulness and worry in a non-clinical sample. Mindfulness, 3(1), 15-21. doi: 10.1007/s12671011-0075-0

Foa, E. B., Huppert, J. D., \& Cahill, S. P. (2006). Emotional Processing Theory: An Update. In Pathological anxiety: Emotional processing in etiology and treatment (pp. 3-24). 
Fox, E., Dutton, K., Yates, A., Georgiou, G. A., \& Mouchlianitis, E. (2015). Attentional Control and Suppressing Negative Thought Intrusions in Pathological Worry. Clinical Psychological Science, 3(4), 593-606. doi: 10.1177/2167702615575878

Freeston, M. H., Dugas, M. J., \& Ladouceur, R. (1996). Thoughts, images, worry, and anxiety. Cognitive Therapy and Research. doi: 10.1007/BF02229237

Geronimi, E. M. C., Szabo-Long, C., Patterson, H., Affrunti, N.W., Richards, A., \& Woodruff-Borden, J. (2015). Understanding Worry in Adolescents: Linguistic Features of Theoretical Worry Models. Poster presented at the Association for Behavioral and Cognitive Therapies Annual Convention, Chicago, IL.

Geronimi, E. M. C., \& Woodruff-Borden, J. (2015). The language of worry: Examining linguistic elements of worry models. Cognition \& Emotion, 29(1-2), 311-318. doi:10.1080/02699931.2014.917071

Greenberg, P. E., Sisitsky, T., Kessler, R. C., Finkelstein, S. N., Berndt, E. R., Davidson, J. R., ... \& Fyer, A. J. (1999). The economic burden of anxiety disorders in the 1990s. Journal of Clinical Psychiatry, 60, 427-435.

Grossman, P. (2008). On measuring nonreactivity in psychosomatic and psychological research. Journal of psychosomatic research, 64(4), 405-408.

Henning, E. R., Turk, C. L., Mennin, D. S., Fresco, D. M., \& Heimberg, R. G. (2007). Impairment and quality of life in individuals with generalized anxiety disorder. Depression and Anxiety, 24, 342-349.

Hirsch, C. R., Hayes, S., Mathews, A., Perman, G., \& Borkovec, T. (2012). The extent and nature of imagery during worry and positive thinking in generalized anxiety disorder. Journal of Abnormal Psychology. doi: 10.1037/a0024947 
Hirsch, C. R., Perman, G., Hayes, S., Eagleson, C., \& Mathews, A. (2015). Delineating the role of negative verbal thinking in promoting worry, perceived threat, and anxiety. Clinical Psychological Science, 3(4), 637-647. doi:

$10.1177 / 2167702615577349$

Hofmann, S. G., Moore, P. M., Gutner, C., \& Weeks, J. W. (2012). Linguistic correlates of social anxiety disorder. Cognition \& Emotion, 26(4), 720-726. doi:10.1080/02699931.2011.602048

Hofmann, S. G., Sawyer, A. T., Witt, A. A., \& Oh, D. (2010). The effect of nonreactivity-based therapy on anxiety and depression: A meta-analytic review. Journal of Consulting and Clinical Psychology, 78(2), 169-183. doi:

\section{$10.1037 / \mathrm{a} 0018555$}

Hoge, E. A., Bui, E., Palitz, S. A., Schwarz, N. R., Owens, M. E., Johnston, J. M., ... Simon, N. M. (2018). The effect of mindfulness meditation training on biological acute stress responses in generalized anxiety disorder. Psychiatry Research, 262(May 2016), 328-332. doi: 10.1016/j.psychres.2017.01.006

Jankowski, T., \& Holas, P. (2014). Metacognitive model of nonreactivity. Consciousness and Cognition, 28(1), 64-80. doi: 10.1016/j.concog.2014.06.005

Kabat-Zinn, J. (2013). Full catastrophe living: Using the wisdom of your body and mind to face stress, pain, and illness. New York: Bantam.

Kabat-Zinn, J., Massion, A. O., Kristeller, J. K., Peterson, L. G., Fletcher, K. E., Pbert, L., ... Santorelli, S. F. (1992). Effectivenes of a Meditation-Based Stress Reduction Program in the Treatment of Anxiety Disorders. American Journal of Psychiatry, 149(7), 936-943. 
Kahn, J. H., Tobin, R. M., Massey, A. E., \& Anderson, J. A. (2007). Measuring emotional expression with the linguistic inquiry and word count. American Journal of Psychology, 120, 263-286.

Kerr, C. E., Sacchet, M. D., Lazar, S. W., Moore, C. I., \& Jones, S. R. (2013).

Nonreactivity starts with the body: somatosensory attention and top-down modulation of cortical alpha rhythms in nonreactivity meditation. Frontiers in Human Neuroscience, 7(12), 1-15. doi: 10.3389/fnhum.2013.00012

Kim, Y. W., Lee, S.-H., Choi, T. K., Suh, S. Y., Kim, B., Kim, C. M., ... Yook, K.-H. (2009). Effectiveness of nonreactivity-based cognitive therapy as an adjuvant to pharmacotherapy in patients with panic disorder or generalized anxiety disorder. Depression and Anxiety, 26(7), 601-606. doi: 10.1002/da.20552

Ladouceur, R., Dugas, M. J., Freeston, M. H., Rhéaume, J., Blais, F., Boisvert, J.-M., ... Thibodeau, N. (1999). Specificity of generalized anxiety disorder symptoms and processes. Behavior Therapy, 30(2), 191-207. doi: 10.1016/S0005-7894(99)80003-3

Ladouceur, R., Gosselin, P., \& Dugas, M. J. (2000). Experimental manipulation of intolerance of uncertainty: A study of a theoretical model of worry. Behaviour Research and Therapy, 38(9), 933-941. doi: 10.1016/S0005-7967(99)00133-3

Lee, S. H., Ahn, S. C., Lee, Y. J., Choi, T. K., Yook, K. H., \& Suh, S. Y. (2007). Effectiveness of a meditation-based stress management program as an adjunct to pharmacotherapy in patients with anxiety disorder. Journal of Psychosomatic Research, 62(2), 189-195. doi: 10.1016/j.jpsychores.2006.09.009

May, R. W., Bamber, M., Seibert, G. S., Sanchez-Gonzalez, M. A., Leonard, J. T., Salsbury, R. A., \& Fincham, F. D. (2016). Understanding the physiology of 
mindfulness: Aortic hemodynamics and heart rate variability. Stress, 19(2), 168174. doi: $10.3109 / 10253890.2016 .1146669$

McLaughlin, K. A., Mennin, D. S., \& Farach, F. J. (2007). The contributory role of worry in emotion generation and dysregulation in generalized anxiety disorder. Behaviour Research and Therapy, 45, 1735-1752. doi: 10.1016/j.brat.2006.12.004

Mennin, D. S., Heimberg, R. G., Turk, C. L., \& Fresco, D. M. (2002). Applying an emotion regulation framework to integrative approaches to generalized anxiety disorder. Clinical Psychology: Science and Practice, 9, 85-90. doi:

\subsection{3/clipsy/9.1.85}

Mennin, D. S., Heimberg, R. G., Turk, C. L., \& Fresco, D. M. (2005). Preliminary evidence for an emotion dysregulation model of generalized anxiety disorder. Behaviour Research and Therapy, 43, 1281-1310. doi: 10.1016/j.brat.2004.08.008

Mennin, D. S., Holaway, R. M., Fresco, D. M., Moore, M. T., \& Heimberg, R. G. (2007). Delineating Components of Emotion and its Dysregulation in Anxiety and Mood Psychopathology. Behavior Therapy, 38, 284-302. doi: 10.1016/j.beth.2006.09.001 Metzger, R. L., Miller, M. L., Cohen, M., Sofka, M., \& Borkovec, T. D. (1990). Worry changes decision making: the effect of negative thoughts on cognitive processing. Journal of Clinical Psychology, 46(1), 78-88. doi: 10.1002/10974679(199001)46:1<78::AID-JCLP2270460113>3.0.CO;2-R

Meyer, T. J., Miller, M. L., Metzger, R. L., \& Borkovec, T. D. (1990). Development and validation of the Penn State Worry Questionnaire. Behaviour Research and Therapy, $28(6), 487-495$. 
Miller, J. J., Fletcher, K., \& Kabat-Zinn, J. (1995). Three-year follow-up and clinical implications of a nonreactivity meditation-based stress reduction intervention in the treatment of anxiety disorders. General Hospital Psychiatry, 17(3), 192-200. doi: $10.1016 / 0163-8343(95) 00025-\mathrm{M}$

Molina, S., Borkovec, T. D., Davey, G. C. L., \& Tallis, F. (1994). The Penn State Worry Questionnaire: Psychometric properties and associated characteristics Worrying: Perspectives on theory, assessment and treatment. (pp. 265-283). Oxford England: John Wiley \& Sons.

Molina, S., Borkovec, T. D., Peasley, C., \& Person, D. (1998). Content analysis of worrisome streams of consciousness in anxious and dysphoric participants. Cognitive Therapy and Research, 22(2), 109-123. doi: 10.1023/A:1018772104808

Moore, S. D., \& Brody, L. R. (2009). Linguistic predictors of nonreactivity in written self-disclosure narratives. Journal of Language and Social Psychology, 28(3), 281296.

Muris, P., Roelofs, J., Meesters, C., \& Boomsma, P. (2004). Rumination and worry in nonclinical adolescents. Cognitive Therapy and Research, 28(4), 539-554. doi: 10.1023/B:COTR.0000045563.66060.3e

Newman, M. G., Llera, S. J., Erickson, T. M., \& Przeworski, A. (2014). Basic science and clinical application of the Contrast Avoidance model in generalized anxiety disorder. Journal of Psychotherapy Integration, 24(3), 155.

Novick-Kline, P., Turk, C. L., Mennin, D. S., Hoyt, E. A., \& Gallagher, C. L. (2005). Level of emotional awareness as a differentiating variable between individuals with 
and without generalized anxiety disorder. Journal of Anxiety Disorders, 19, 557572. doi: 10.1016/j.janxdis.2004.06.001

O'brien, R. M. (2007). A caution regarding rules of thumb for variance inflation factors. Quality \& quantity, 41(5), 673-690.

Peasley-Miklus, C., \& Vrana, S. R. (2000). Effect of worrisome and relaxing thinking on fearful emotional processing. Behaviour Research and Therapy, 38(2), 129-144. doi: 10.1016/S0005-7967(99)00025-X

Pennebaker, J. W., Chung, C. K., Ireland, M., Gonzales, A., \& Booth, R. J. (2007). The development and psychometric properties of LIWC2007. Austin, TX: LIWC Inc.

Querstret, D., \& Cropley, M. (2013). Assessing treatments used to reduce rumination and/or worry: A systematic review. Clinical Psychology Review, 33(8), 996-1009. doi: 10.1016/j.cpr.2013.08.004

Robichaud, M., Dugas, M. J., \& Conway, M. (2003). Gender differences in worry and associated cognitive-behavioral variables. Journal of anxiety disorders, 17(5), 501516.

Roemer, L., \& Orsillo, S. M. (2002). Expanding our conceptualizations of and treatment for generalized anxiety disorder: Integrating nonreactivity/acceptance-based approaches with existing cognitive behavioral models. Clinical Psychology: Science and Practice, 1, 54-68.

Roemer, L., \& Orsillo, S. M. (2007). An Open Trial of an Acceptance-Based Behavior Therapy for Generalized Anxiety Disorder. Behavior Therapy, 38(1), 72-85. doi: 10.1016/j.beth.2006.04.004 
Roemer, L., Orsillo, S. M., \& Salters-Pedneault, K. (2008). Efficacy of an acceptancebased behavior therapy for generalized anxiety disorder: evaluation in a randomized controlled trial. Journal of Consulting and Clinical Psychology, 76(6), 1083-1089. doi: $10.1037 / \mathrm{a} 0012720$

Rude, S., Gortner, E.-M., \& Pennebaker, J. (2004). Language use of depressed and depression-vulnerable college students. Cognition \& Emotion, 18(8), 1121-1133. doi: $10.1080 / 02699930441000030$

Ruiz, F. J. (2014). The relationship between low levels of nonreactivity skills and pathological worry: The mediating role of psychological inflexibility, 30(2004), $887-897$.

Ruscio, A. M., \& Borkovec, T. D. (2004). Experience and appraisal of worry among high worriers with and without generalized anxiety disorder. Behaviour Research and Therapy, 42(12), 1469-1482. doi: 10.1016/j.brat.2003.10.007

Russell, M., \& Davey, G. C. L. (1993). The relationship between life event measures and anxiety and its cognitive correlates. Personality and Individual Differences, 14(2), 317-322. doi: 10.1016/0191-8869(93)90129-Q

Salters-Pedneault, K., Roemer, L., Tull, M. T., Rucker, L., \& Mennin, D. S. (2006). Evidence of broad deficits in emotion regulation associated with chronic worry and generalized anxiety disorder. Cognitive Therapy and Research, 30, 469-480. doi: $10.1007 / \mathrm{s} 10608-006-9055-4$

Sass, S. M., Berenbaum, H., \& Abrams, E. M. (2013). Discomfort with emotion moderates distress reduction in a brief nonreactivity intervention. International 
Journal of Behavioral Consultation and Therapy, 7(4), 24-27. doi:

$10.1037 / \mathrm{h} 0100962$

Segal, Z. V., Williams, J. M. G., \& Teasdale, J. D. (2012). Nonreactivity-based cognitive therapy for depression. Guilford Press.

Silva, R. (2014). Pensamento e linguagem. Lisbon: Centro de Filosofia da Universidade de Lisboa.

Stirman, S. W., \& Pennebaker, J. W. (2001). Word use in the poetry of suicidal and nonsuicidal poets. Psychosomatic Medicine, 63(4), 517-522.

Tallis, F., Davey, G. C. L., \& Capuzzo, N. (1994). The phenomenology of nonpathological worry: A preliminary investigation. In G. C. L. Davey \& F. Tallis (Eds.), Worrying: Perspectives on theory, assessment and treatment (pp. 61-89). New York: Wiley.

Tallis, F., \& Eysenck, M. W. (1994). Worry: Mechanisms and Modulating Influences. Behavioural and Cognitive Psychotherapy. doi: 10.1017/S1352465800011796

Tang, A. Y., Ma, Y., Fan, Y., Feng, H., Wang, J., Feng, S., .. Fan, M. (2009). Central and autonomic nervous system interaction is altered by short-term meditation. Proceedings of the national Academy of Sciences of the United States of America,106(22), 8865-8870. doi: 10.1073/pnas.0904031106

Tausczik, Y. R., \& Pennebaker, J. W. (2010). The psychological meaning of words: LIWC and computerized text analysis methods. Journal of Language and Social Psychology, 29, 24-54.

Teasdale, J. D., Moore, R. G., Hayhurst, H., Pope, M., Williams, S., \& Segal, Z. V. (2002). Metacognitive awareness and prevention of relapse in depression: empirical 
evidence. Journal of Consulting and Clinical Psychology, 70(2), 275-287. doi: 10.1037/0022-006X.70.2.275

Thayer, J. F., Friedman, B. H., \& Borkovec, T. D. (1996). Autonomic characteristics of generalized anxiety disorder and worry. Biological Psychiatry, 39(4), 255-266. doi: $10.1016 / 0006-3223(95) 00136-0$

Thera, N. (1992). The heart of Buddhist meditation . Kandy: Buddhist Publication Society.

Thielsch, C., Andor, T., \& Ehring, T. (2015). Do Metacognitions and Intolerance of Uncertainty Predict Worry in Everyday Life? An Ecological Momentary Assessment Study. Behavior Therapy, 46(4), 532-543. doi:

10.1016/j.beth.2015.05.001

Turk, C. L., Heimberg, R. G., Luterek, J. A., Mennin, D. S., \& Fresco, D. M. (2005). Emotion dysregulation in generalized anxiety disorder: A comparison with social anxiety disorder. Cognitive Therapy and Research, 29(1), 89-106. doi: $10.1007 / \mathrm{s} 10608-005-1651-1$

Vlemincx, E., Vigo, D., Vansteenwegen, D., Van den Bergh, O., \& Van Diest, I. (2013). Do not worry, be mindful: Effects of induced worry and nonreactivity on respiratory variability in a nonanxious population. International Journal of Psychophysiology, 87(2), 147-151. doi: 10.1016/j.ijpsycho.2012.12.002

Wells, A. (1994). A multi-dimensional measure of worry: Development and preliminary validation of the anxious thoughts inventory. Anxiety, Stress \& Coping. doi: $10.1080 / 10615809408248803$ 
Wells, A. (1995). Meta-Cognition and Worry: A Cognitive Model of Generalized Anxiety Disorder. Behavioural and Cognitive Psychotherapy. doi: $10.1017 / \mathrm{S} 1352465800015897$

Wells, A. (1999). A cognitive model of generalized anxiety disorder. Behavior Modification, 23(4), 526-555. doi: 10.1177/0145445599234002

Wells, A. (2005). The metacognitive model of GAD: Assessment of meta-worry and relationship with DSM-IV generalized anxiety disorder. Cognitive Therapy and Research, 29(1), 107-121. doi: 10.1007/s10608-005-1652-0

Wells, A. (2006). The Metacognitive Model of Worry and generalized anxiety disorder. In: G. Davey \& A. Wells (Eds.), Worry and its psychological disorders: theory, assessment and treatment (pp. 179-200). West Sussex, England: Wiley \& Sons.

Wells, A., \& Carter, K. (1999). Preliminary tests of a cognitive model of Generalised Anxiety Disorder. Behaviour Research and Therapy, 37, 585-594.

Wells, A., \& Carter, K. (2001). Further tests of a cognitive model of generalized anxiety disorder: Metacognitions and worry in GAD, panic disorder, social phobia, depression, and nonpatients. Behavior Therapy, 32(1), 85-102. doi: 10.1016/S00057894(01)80045-9

Wittchen, H.-U., Zhao, S., Kessler, R. C., \& Eaton, W. W. (1994). DSM-III-R generalized anxiety disorder in the National Comorbidity Survey. Archives of General Psychiatry, 51, 355-364.

Yang, Z., Wang, R., Chen, H., \& Ding, J. (2015). Personality and worry: The role of intolerance of uncertainty. Social Behavior and Personality, 43(10), 1607-1616. doi: 10.2224/sbp.2015.43.10.1607 


\section{Table 1}

$\underline{\text { Participant Demographics }}$

\begin{tabular}{lrr}
\hline Variable/Level & $N$ & $\%$ \\
\hline Ethnicity & & \\
$\quad$ European American & 41 & 89.13 \\
African American & 3 & 6.52 \\
Other & 2 & 4.34 \\
Education & & \\
$\quad$ High School Diploma & 2 & 4.35 \\
Some college & 5 & 10.87 \\
Currently in college or graduate school & 3 & 6.32 \\
Associate's or equivalent & 1 & 2.17 \\
Bachelor's Degree & 16 & 34.78 \\
Masters Degree & 11 & 23.91 \\
Doctorate & 8 & 17.39 \\
Income & & \\
\$10,000 - \$19,000 & 1 & 2.17 \\
\$30,000 - \$39,999 & 2 & 4.35 \\
\$40,000 - \$49,999 & 6 & 13.04 \\
\$50,000 - \$59,999 & 2 & 4.35 \\
Over \$60,000 & 35 & 76.09 \\
\hline
\end{tabular}


Table 2

Descriptive Statistics of Self-Report and Language Measures

\begin{tabular}{lcc}
\hline Measure & Mean & SD \\
\hline Worry & 47.72 & 15.97 \\
Mindfulness & 132.17 & 19.82 \\
W. Present tense & 16.11 & 4.06 \\
W. Number of words & 314.43 & 121.28 \\
W. Questions & 1.34 & .50 \\
W. Anxiety & 1.20 & .68 \\
W. Negative emotion & 1.75 & .61 \\
N. Present tense & 7.98 & 3.86 \\
N. Number of words & 304.39 & 108.37 \\
N. Questions & 1.32 & .74 \\
N. Anxiety & .39 & .47 \\
N. Negative emotion & .94 & .58 \\
\hline Note. W. & & \\
\hline
\end{tabular}

Note. W. = worry condition; N. = neutral condition. 


\section{Table 3}

Correlation Coefficients for Worry and Mindfulness Variables

\begin{tabular}{|c|c|c|c|c|c|c|c|}
\hline & 1. & 2. & 3. & 4. & 5. & 6. & 7. \\
\hline \multirow[t]{2}{*}{ 1. Worry } & --- & - & -.227 & -.276 & $-.304 *$ & $.512 * * *$ & $.609 * * *$ \\
\hline & & $.554 * *$ & & & & & \\
\hline 2. Mindfulness & & --- & $.518 * * *$ & $.789 * * *$ & $.750 * * *$ & $.532 * * *$ & $.786 * * *$ \\
\hline 3. Observing & & & --- & .278 & .224 & -.105 & $.291 *$ \\
\hline 4. Describing & & & & --- & $.512 * * *$ & $.308^{*}$ & $.519 * * *$ \\
\hline 5. Acting with & & & & & --- & $.294^{*}$ & $.486 * * *$ \\
\hline \multicolumn{8}{|l|}{ awareness } \\
\hline 6. Nonjudgement & & & & & & --- & $.402 * *$ \\
\hline 7. Nonreactivity & & & & & & & --- \\
\hline
\end{tabular}

Note. ${ }^{*} \mathrm{p}<.05 ; * * \mathrm{p}<.01 ; * * * \mathrm{p}<.001$; Observing, describing, acting with awareness, nonjudgement, and nonreactivity are part of the mindfulness measure used (i.e., FFMQ). 


\section{Table 4}

Hierarchical Multiple Regression Predicting Worry from Language Variables

\begin{tabular}{lcccrr}
\hline & $B$ & $S E B$ & $\beta$ & $t$ & $p$ \\
\hline Model 1 & & & & & \\
Constant & 88.66 & 16.34 & & 5.42 & $<.001$ \\
Age & -1.01 & .41 & -.36 & -2.47 & .018 \\
Model 2 & & & & & \\
Constant & 90.04 & 17.24 & & 5.22 & $<.001$ \\
Age & -.80 & .39 & -.28 & -2.04 & .048 \\
NE Words & 1.57 & 6.73 & .06 & .23 & .817 \\
Anxiety- & -10.61 & 6.11 & -.46 & -1.74 & .090 \\
related words & & & & & \\
\hline
\end{tabular}

Note. $\mathrm{NE}=$ negative emotion. 
Table 5

Hierarchical Multiple Regression Predicting Nonreactivity from Language Variables

\begin{tabular}{cccccc}
\hline & $B$ & $S E B$ & $\beta$ & $t$ & $p$ \\
\hline Constant & 16.85 & 2.64 & & 6.37 & $<.001$ \\
NE Words & 2.83 & 2.54 & .32 & 1.11 & .271 \\
$\begin{array}{c}\text { Anxiety- } \\
\text { related words }\end{array}$ & .063 & 2.26 & .01 & .028 & .978 \\
\hline
\end{tabular}

Note. $\mathrm{NE}=$ negative emotion. 
Table 6

Hierarchical Multiple Regression Predicting Nonjudgement from Language Variables

\begin{tabular}{cccccc}
\hline & $B$ & $S E B$ & $\beta$ & $t$ & $p$ \\
\hline Constant & 26.77 & 2.55 & & 10.51 & $<.001$ \\
NE Words & -1.11 & 2.45 & -.13 & -.45 & .651 \\
$\begin{array}{c}\text { Anxiety- } \\
\text { related words }\end{array}$ & 3.42 & 2.18 & .44 & 1.57 & .125 \\
\hline
\end{tabular}

Note. $\mathrm{NE}=$ negative emotion. 

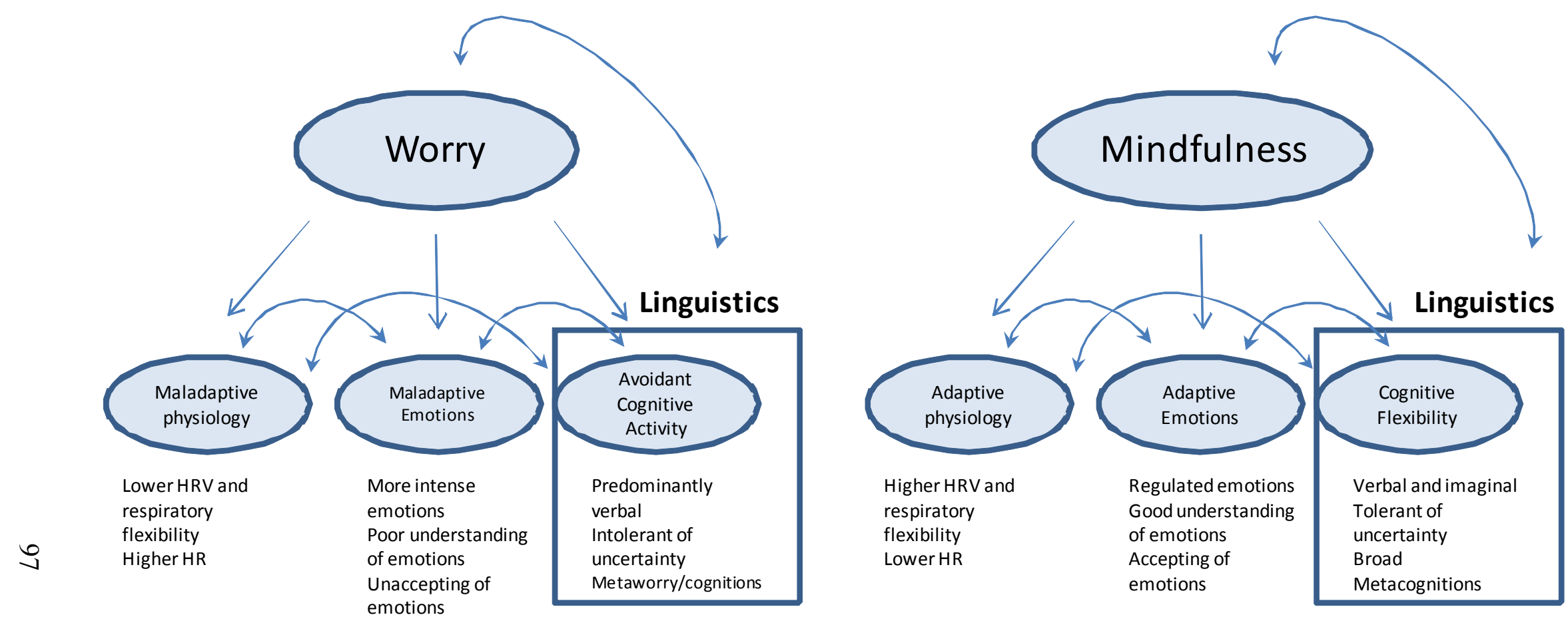

Figure 1. Worry and mindfulness features. 


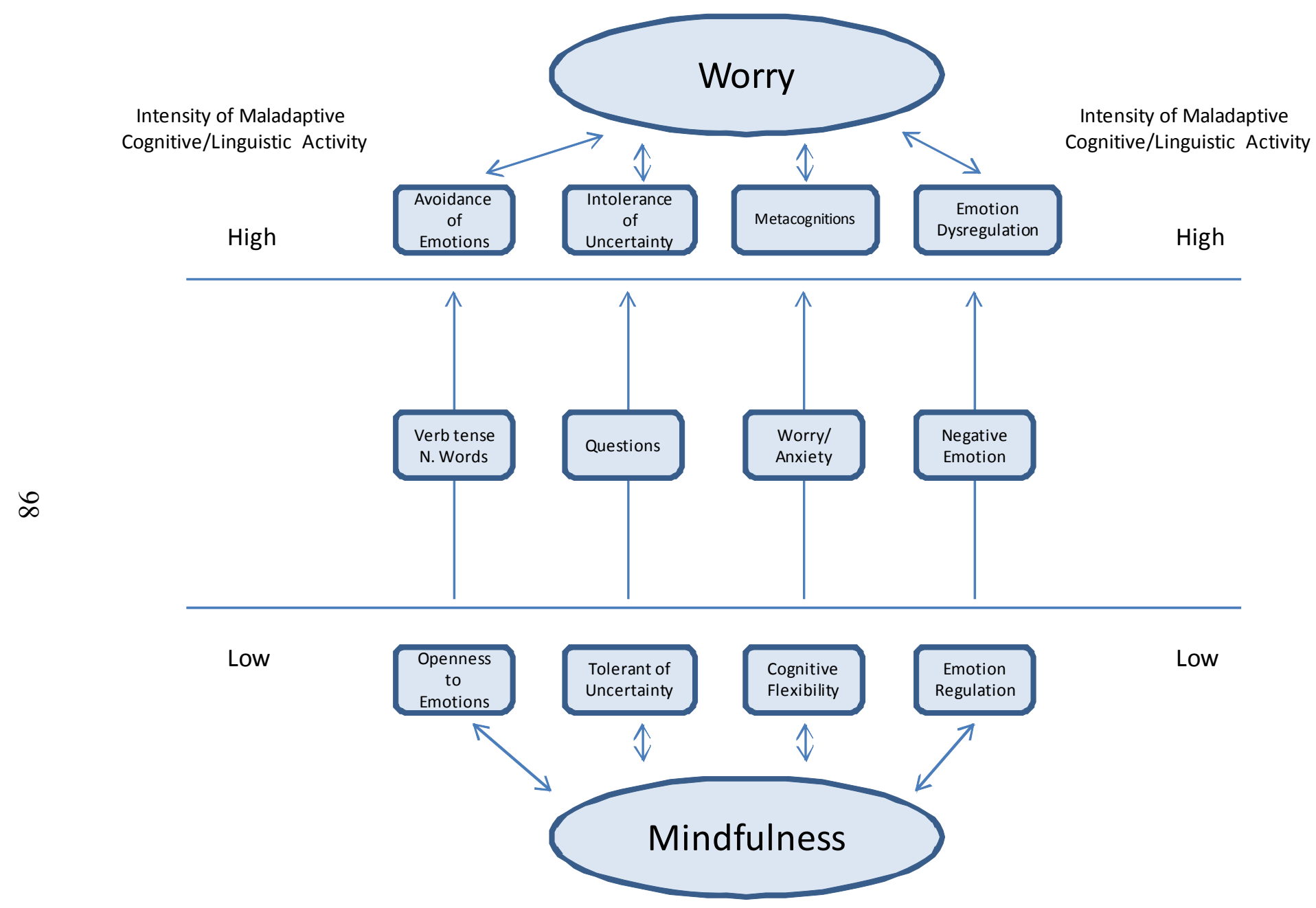

Figure 2. Linguistic elements contributing to the "experiential barrier" 


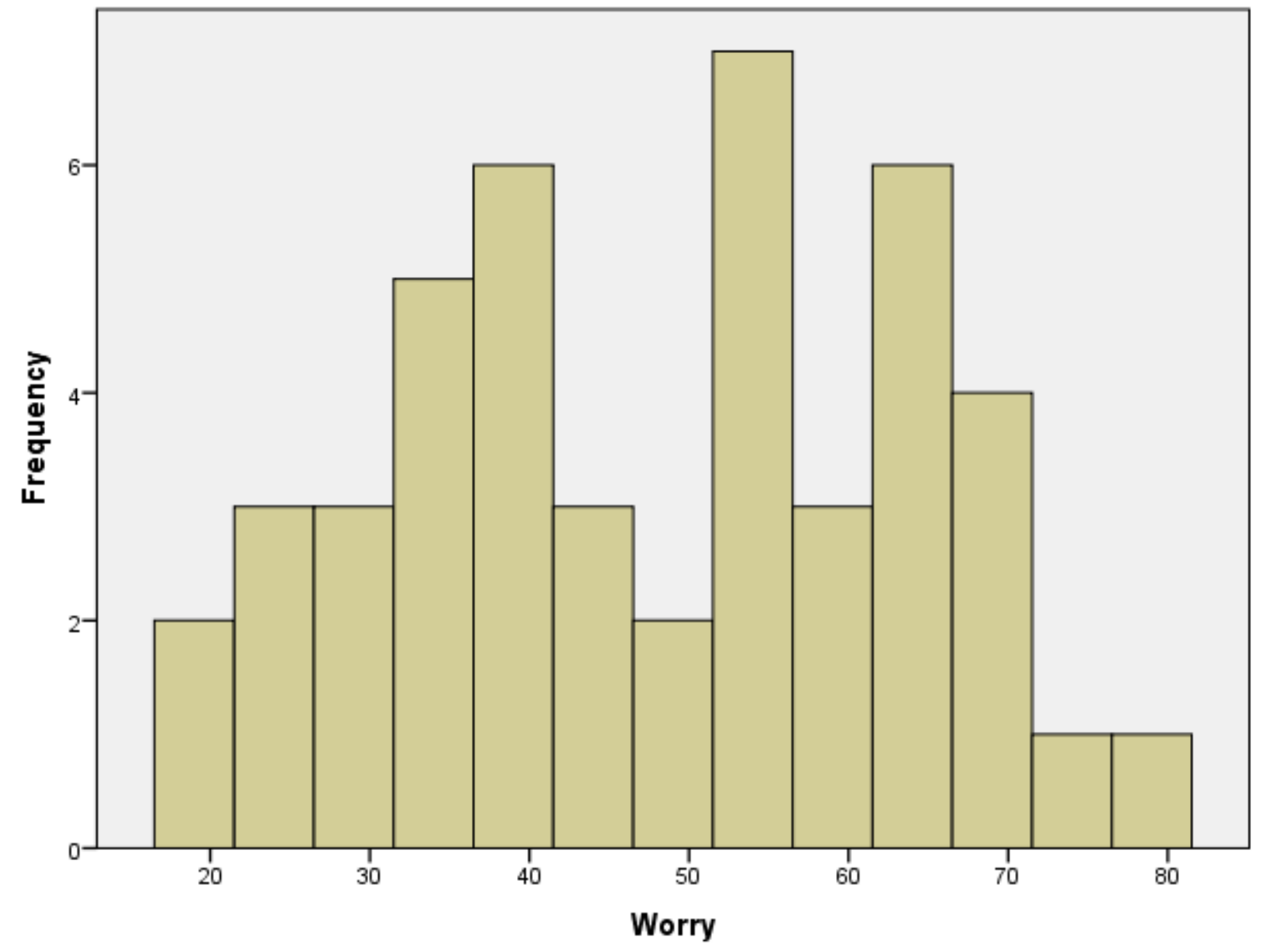

Figure 3. Worry histogram. 


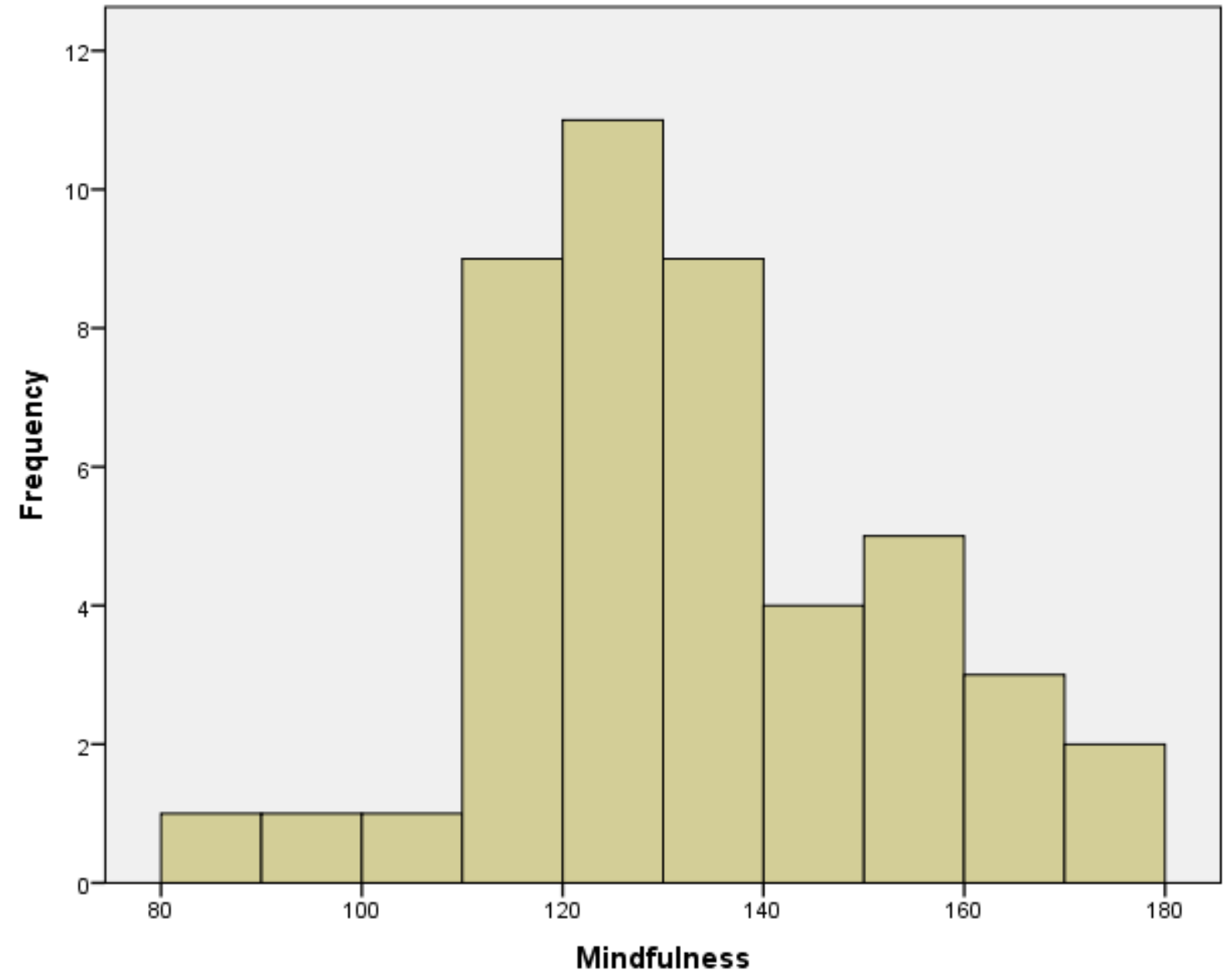

Figure 4. Mindfulness histogram. 


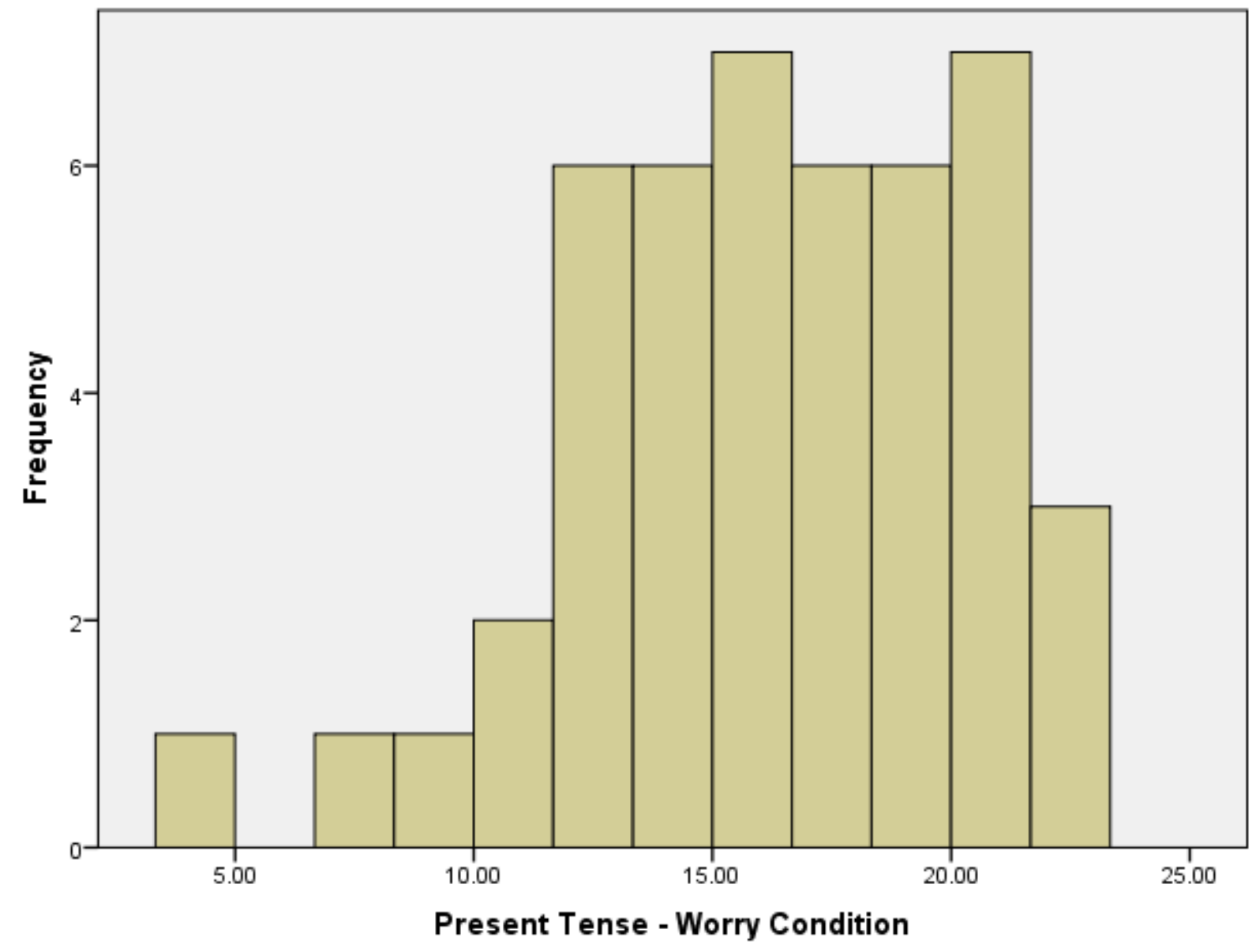

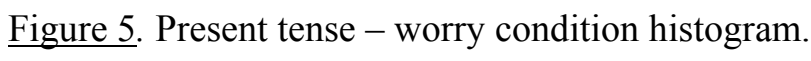




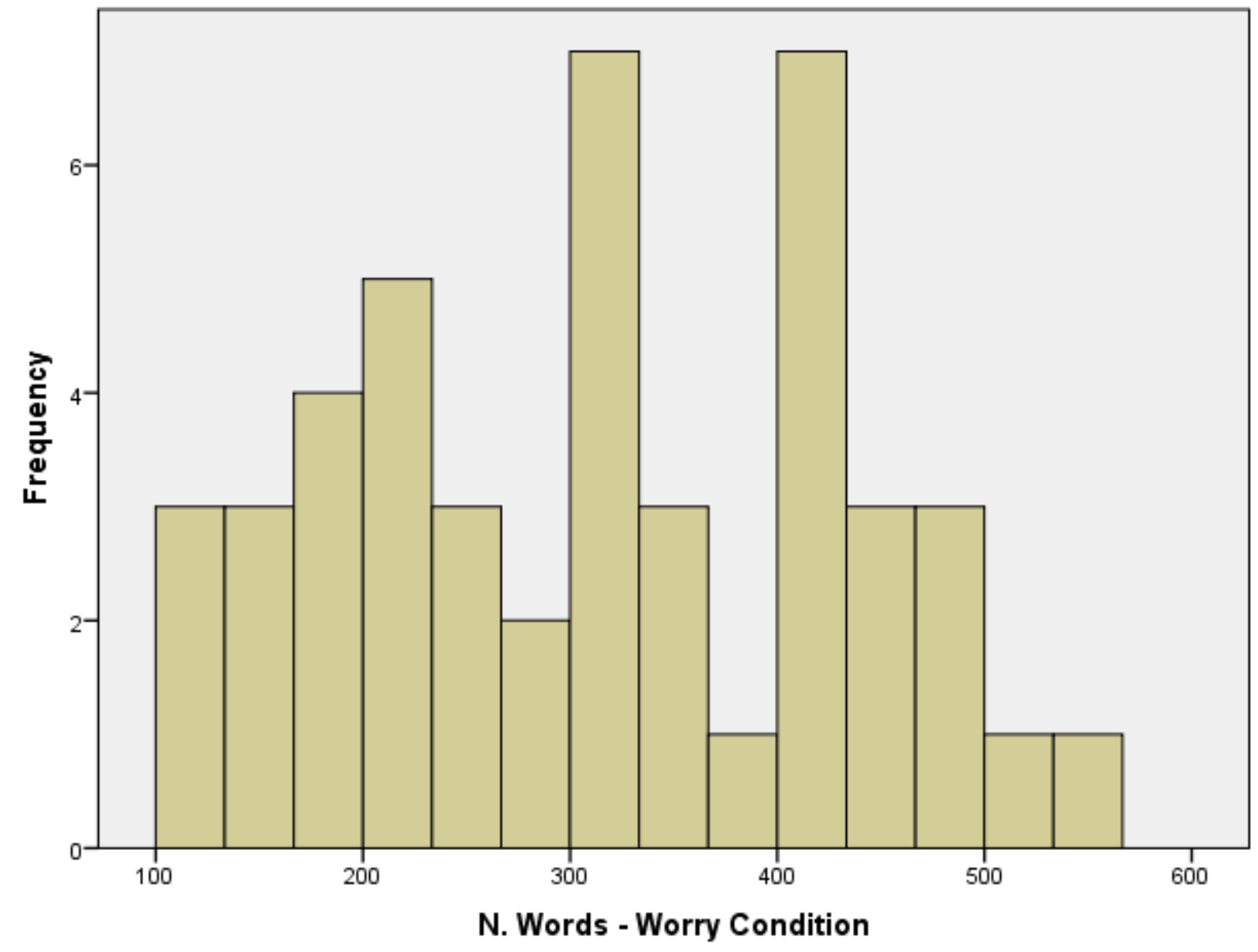

Figure 6. Number of words - worry condition histogram. 


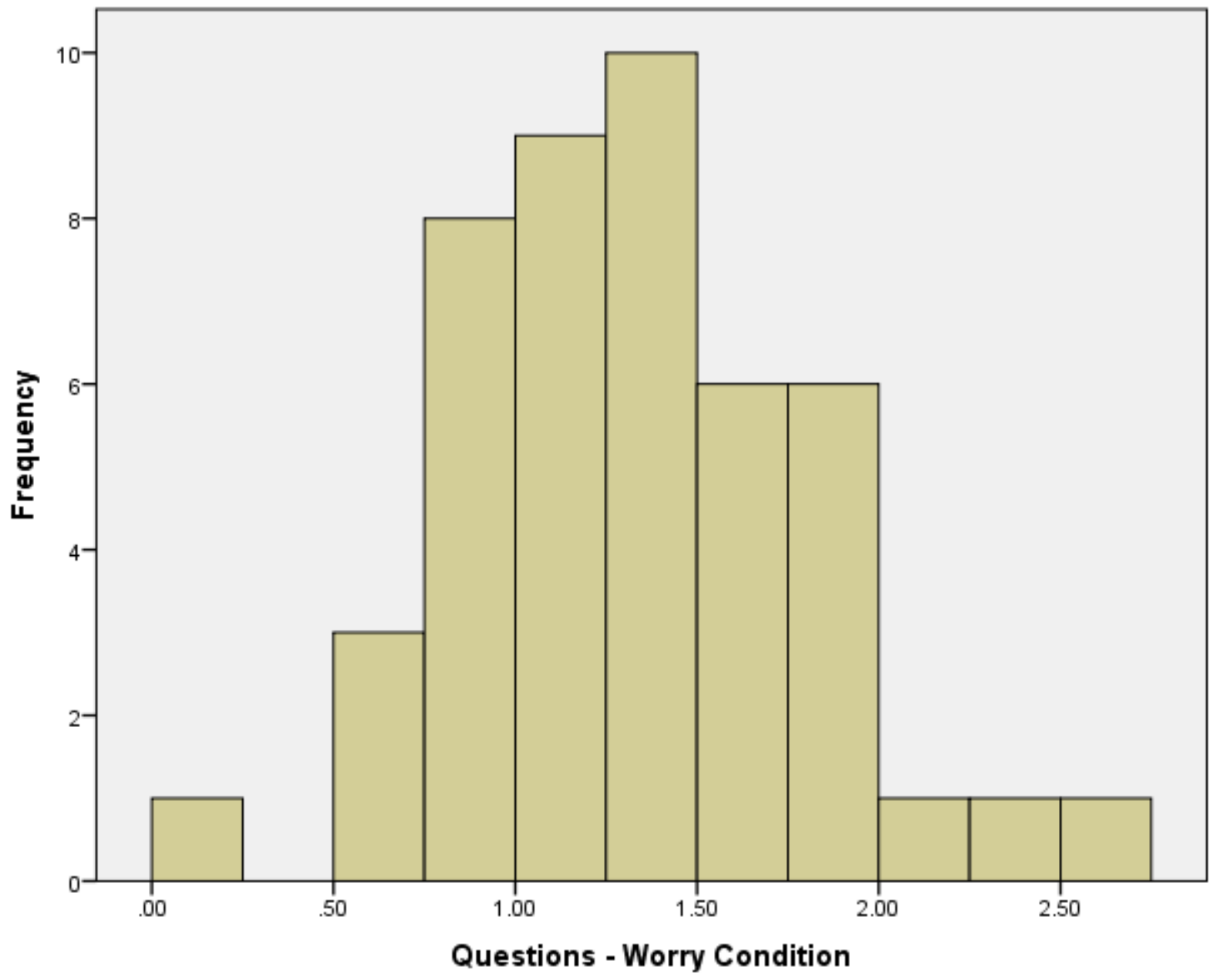

Figure 7. Questions - worry condition histogram. 


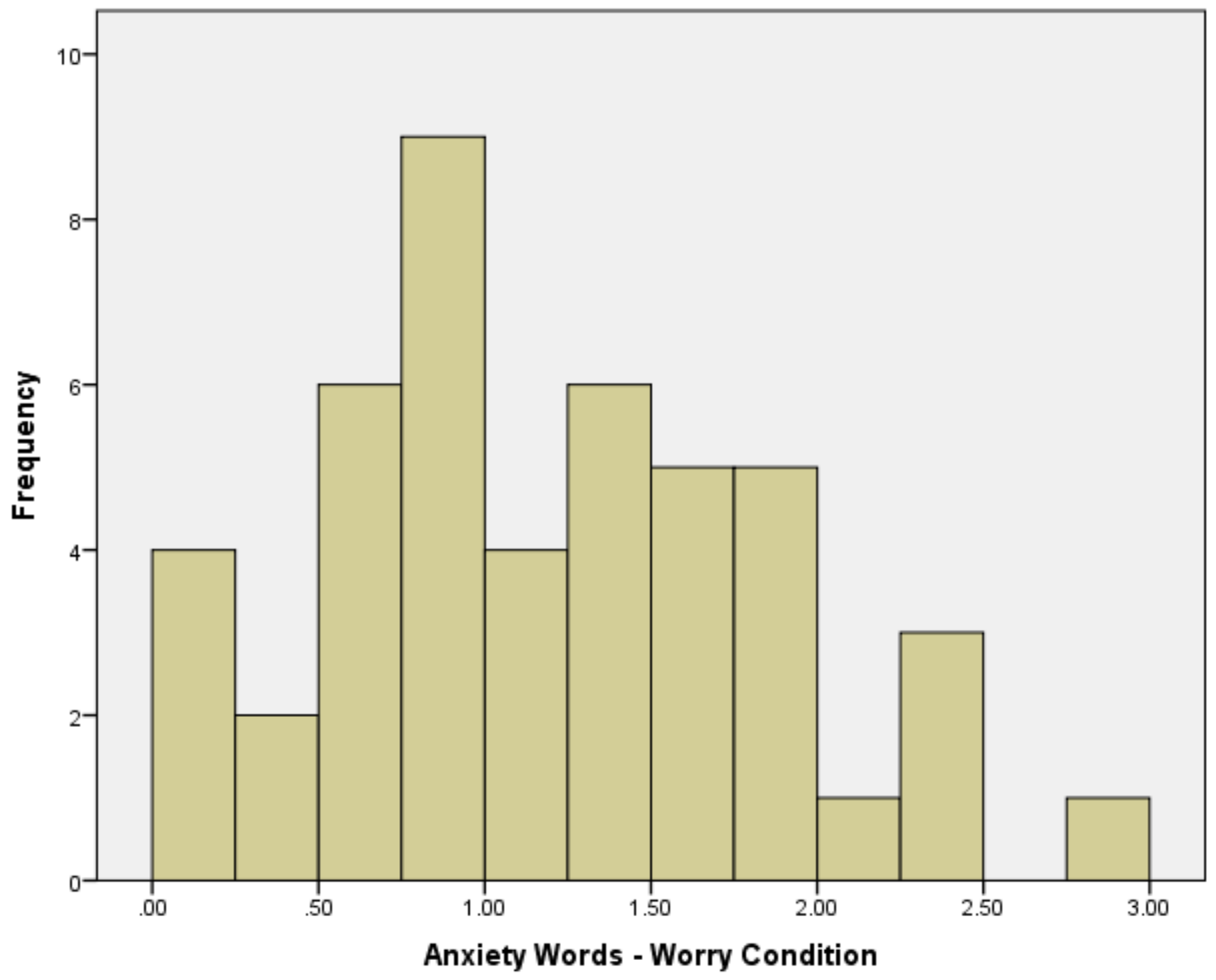

Figure 8. Anxiety related words - worry condition histogram. 


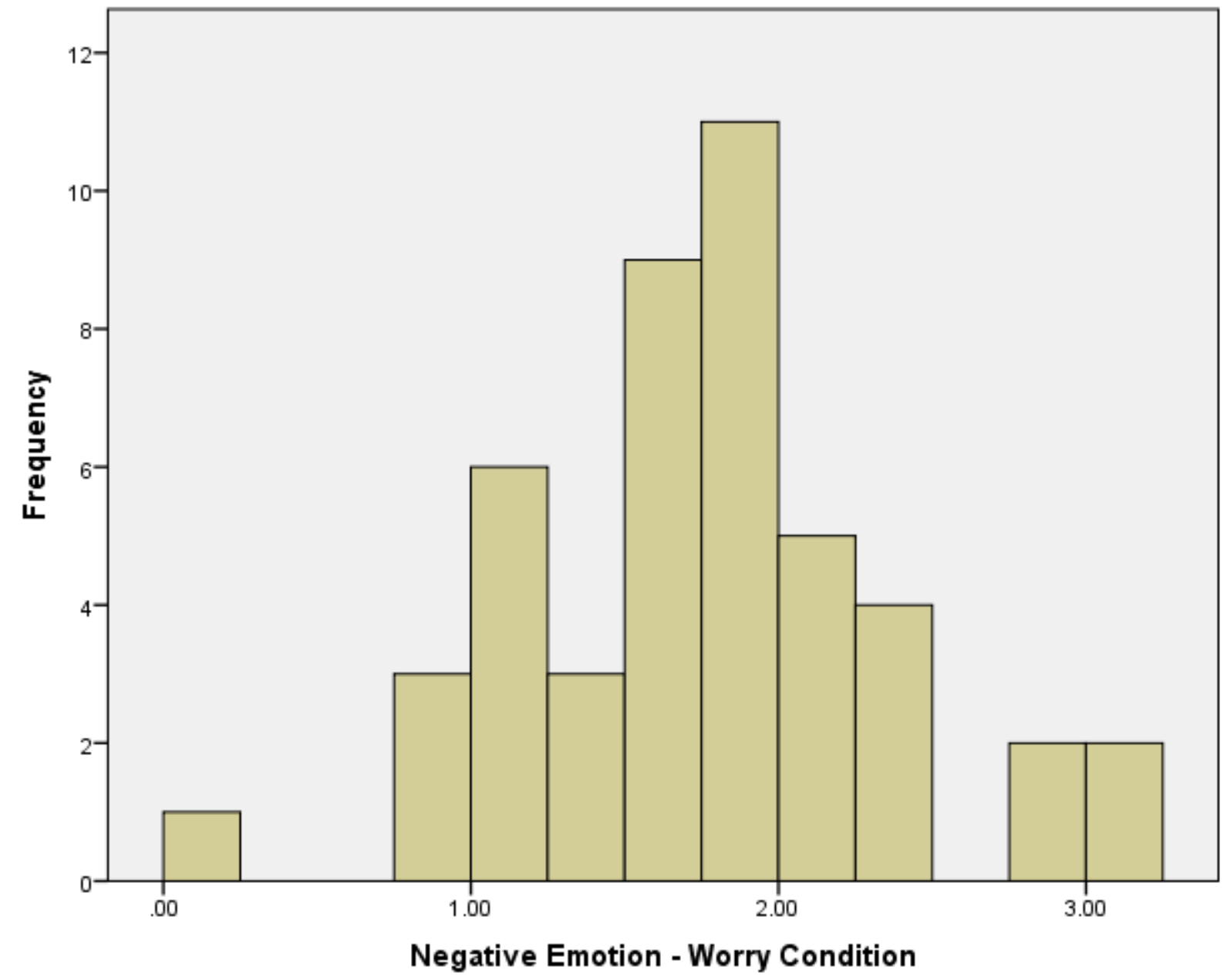

Figure 9. Negative emotion words - worry condition histogram. 


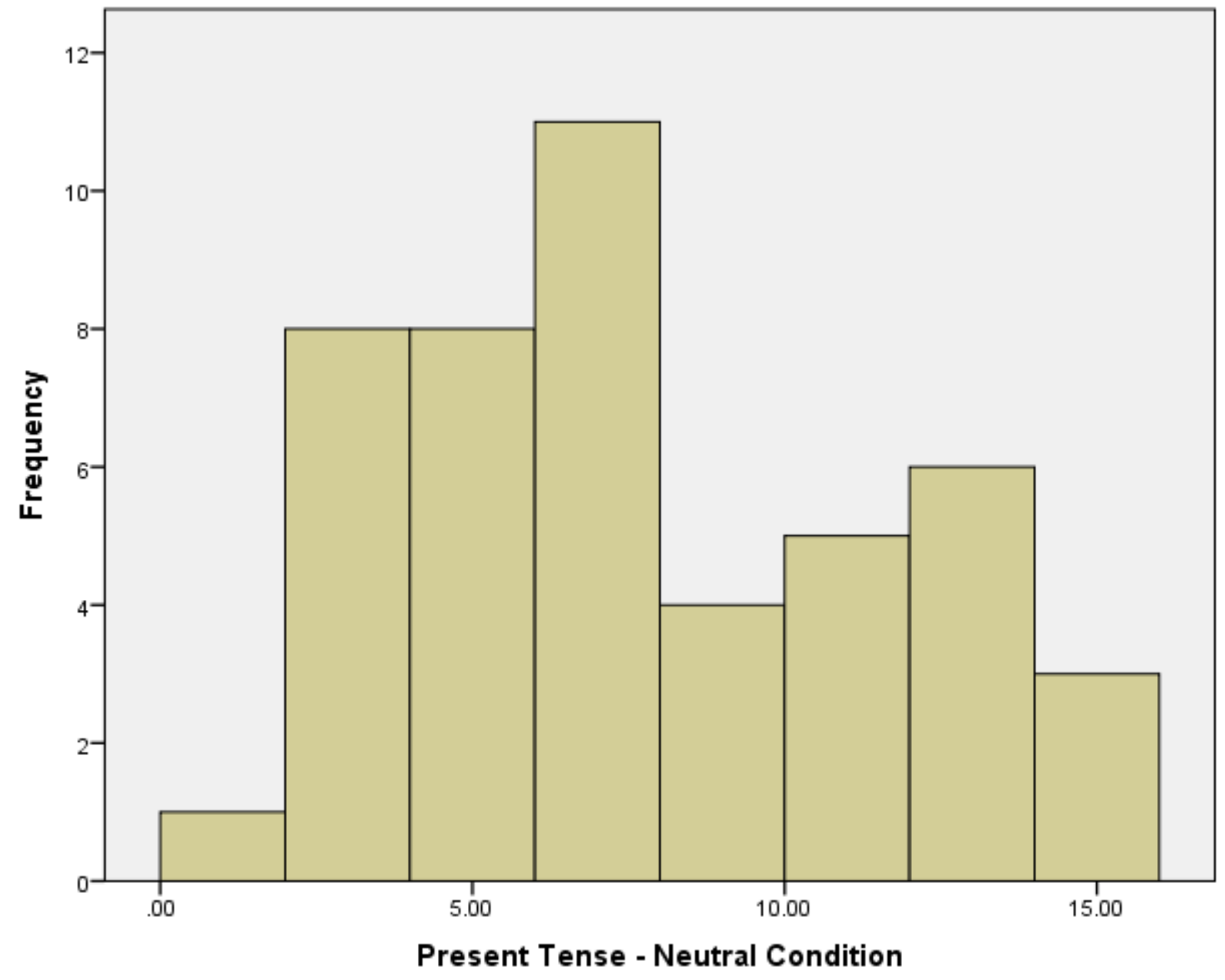

Figure 10. Present tense - neutral condition histogram. 


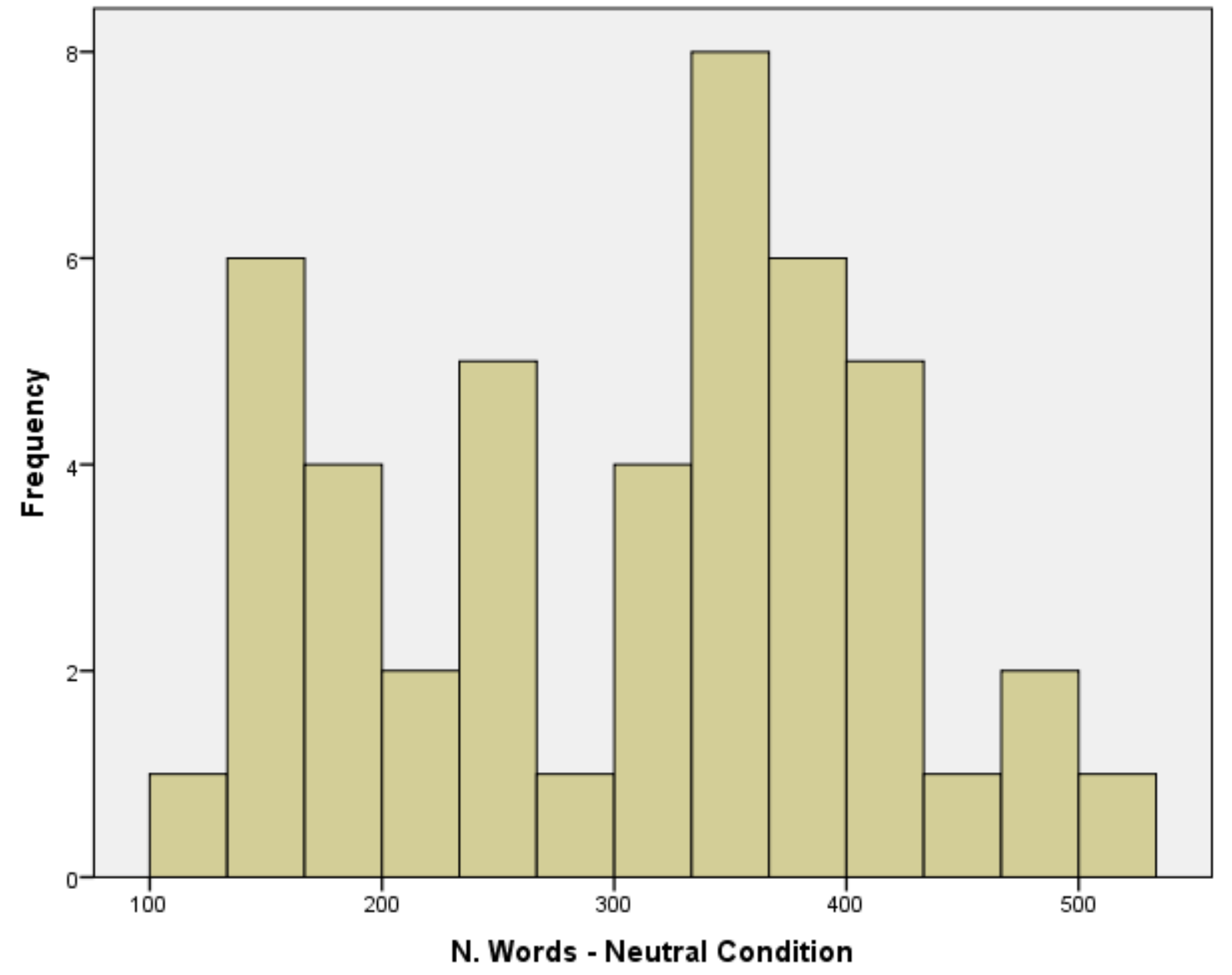

Figure 11. Number of words - neutral condition histogram. 


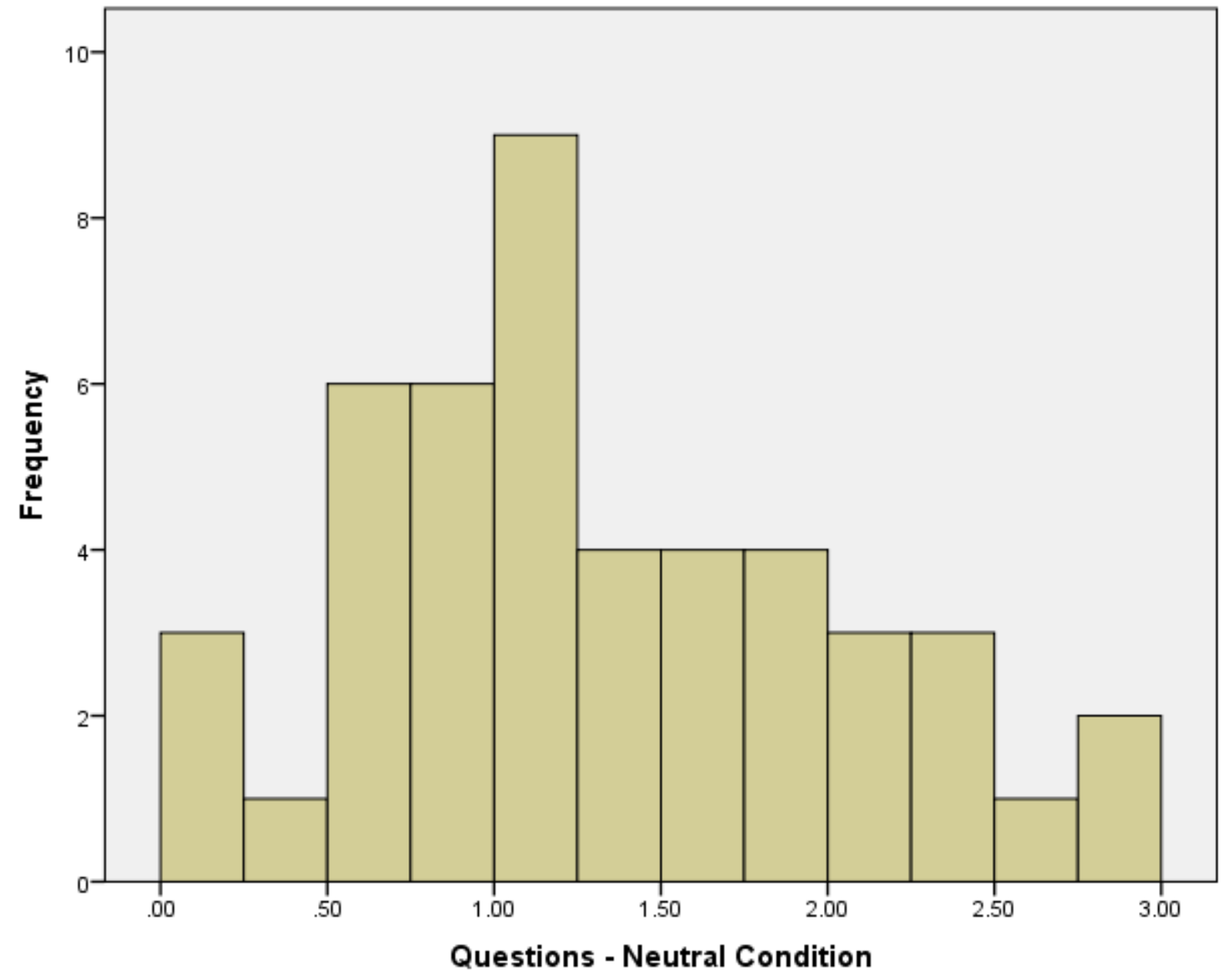

Figure 12. Number of questions - neutral condition histogram. 


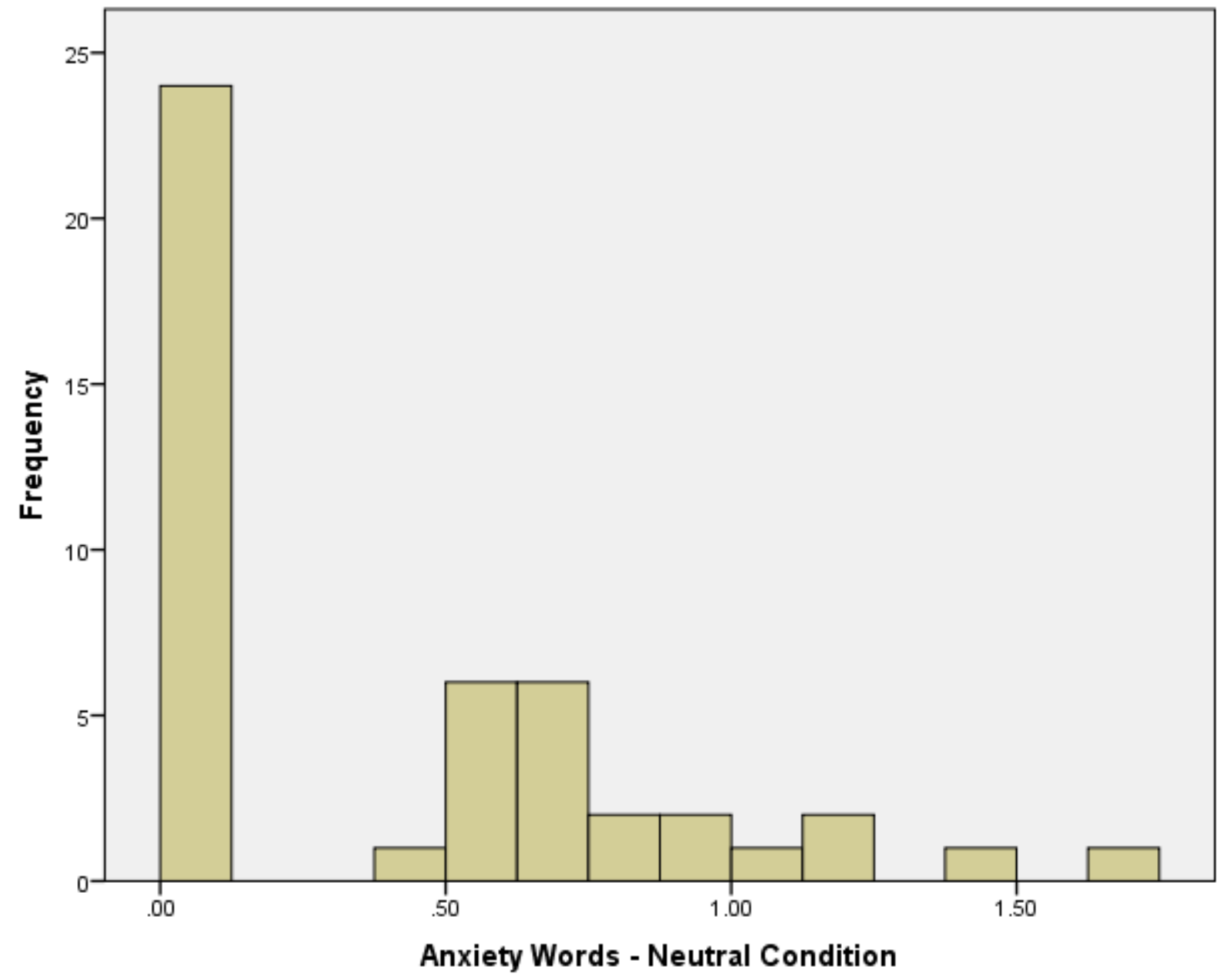

Figure 13. Anxiety words - neutral condition histogram. 


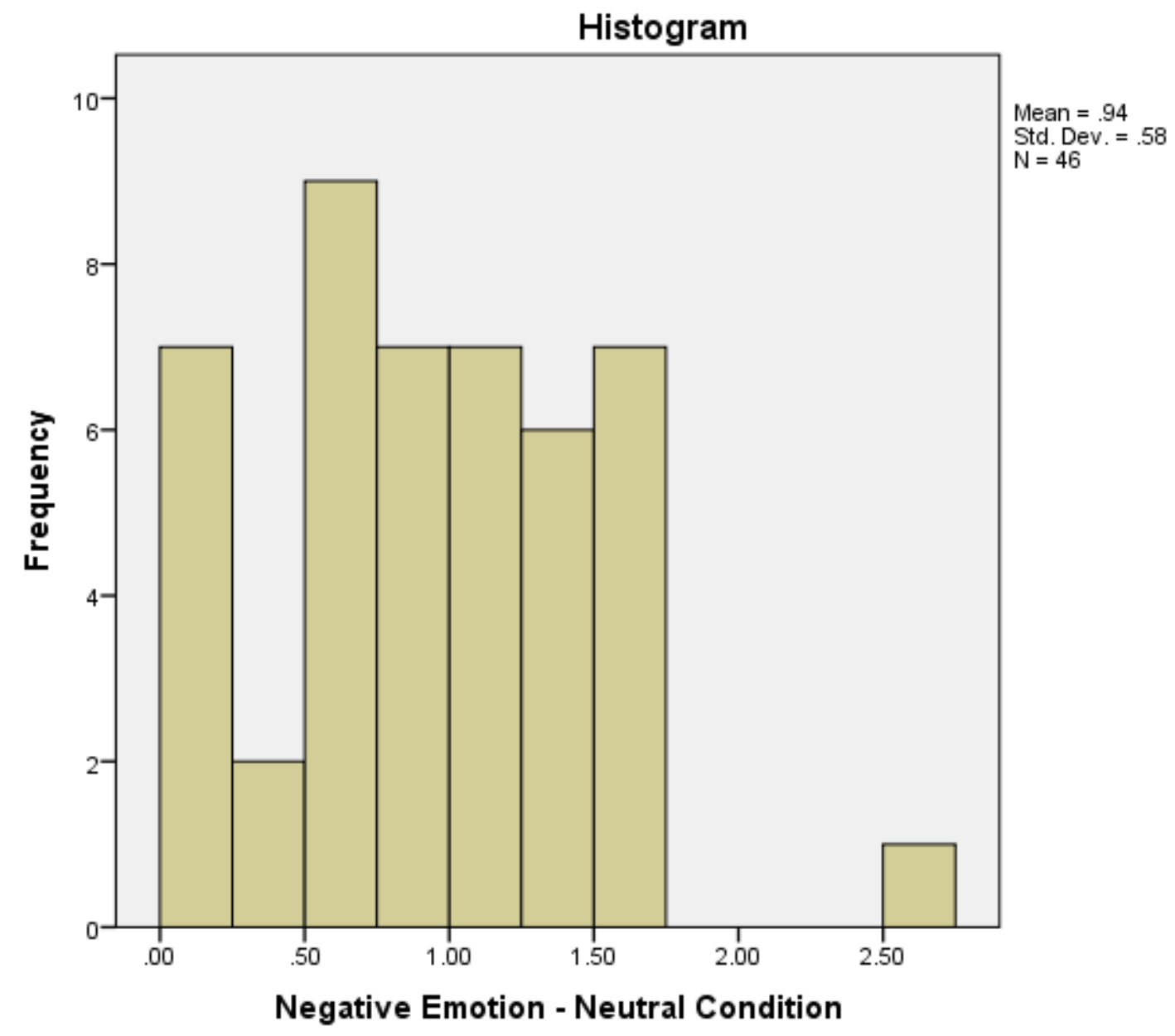

Figure 14. Negative emotion words - neutral condition histogram. 


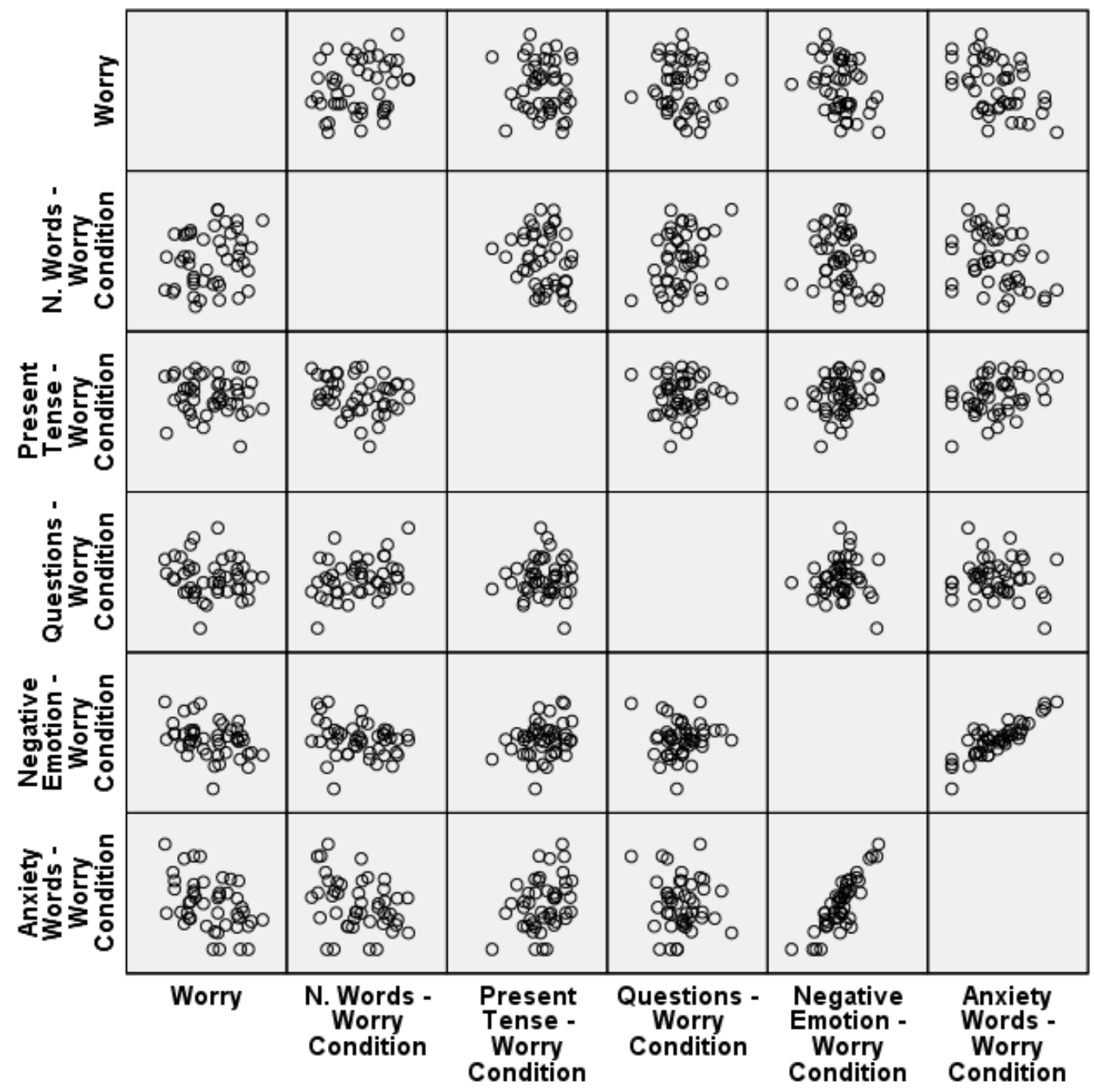

Figure 15. Worry and language scatterplotts - worry condition. 


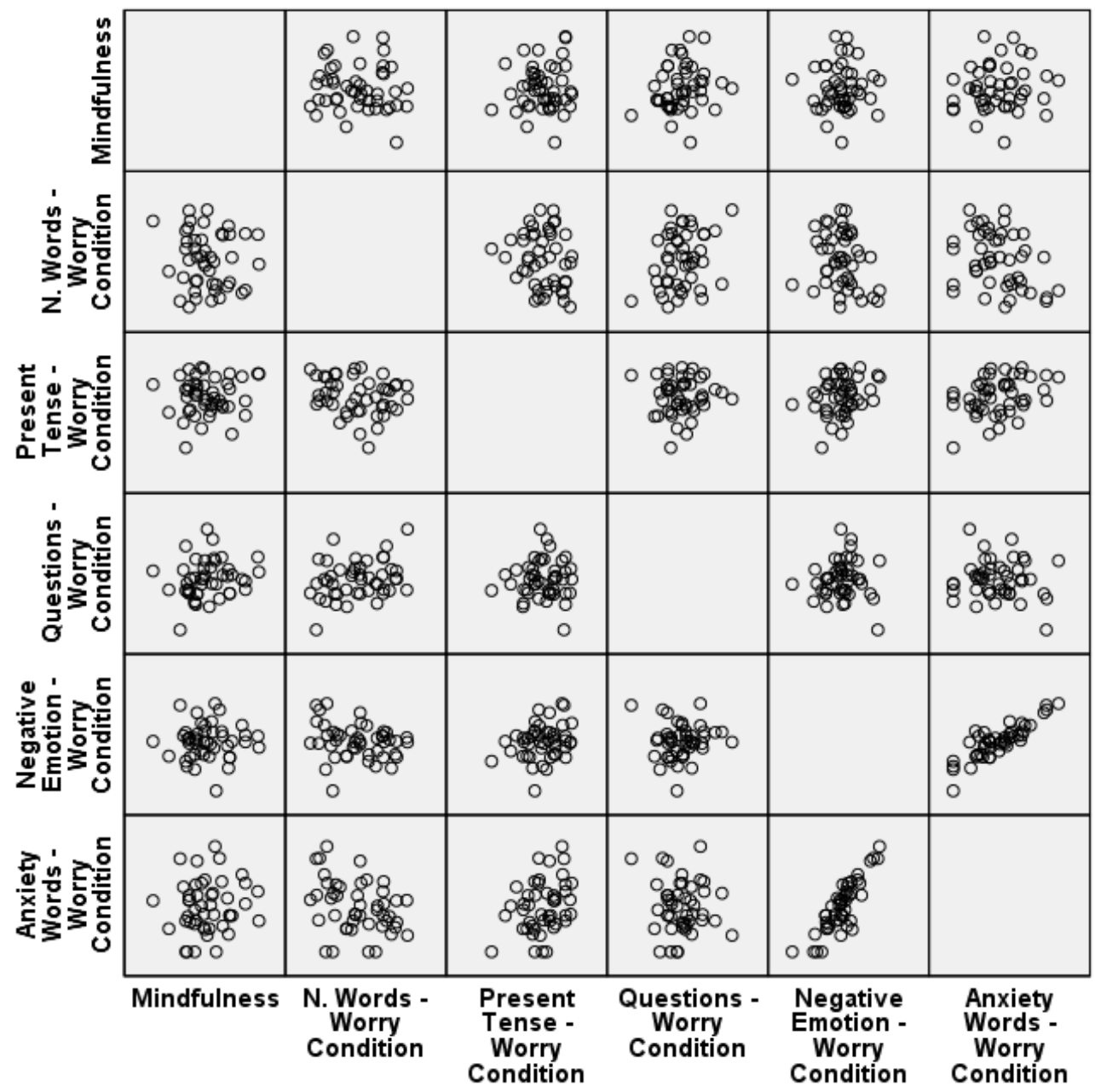

Figure 16. Mindfulness and language scatterplots - worry condition. 


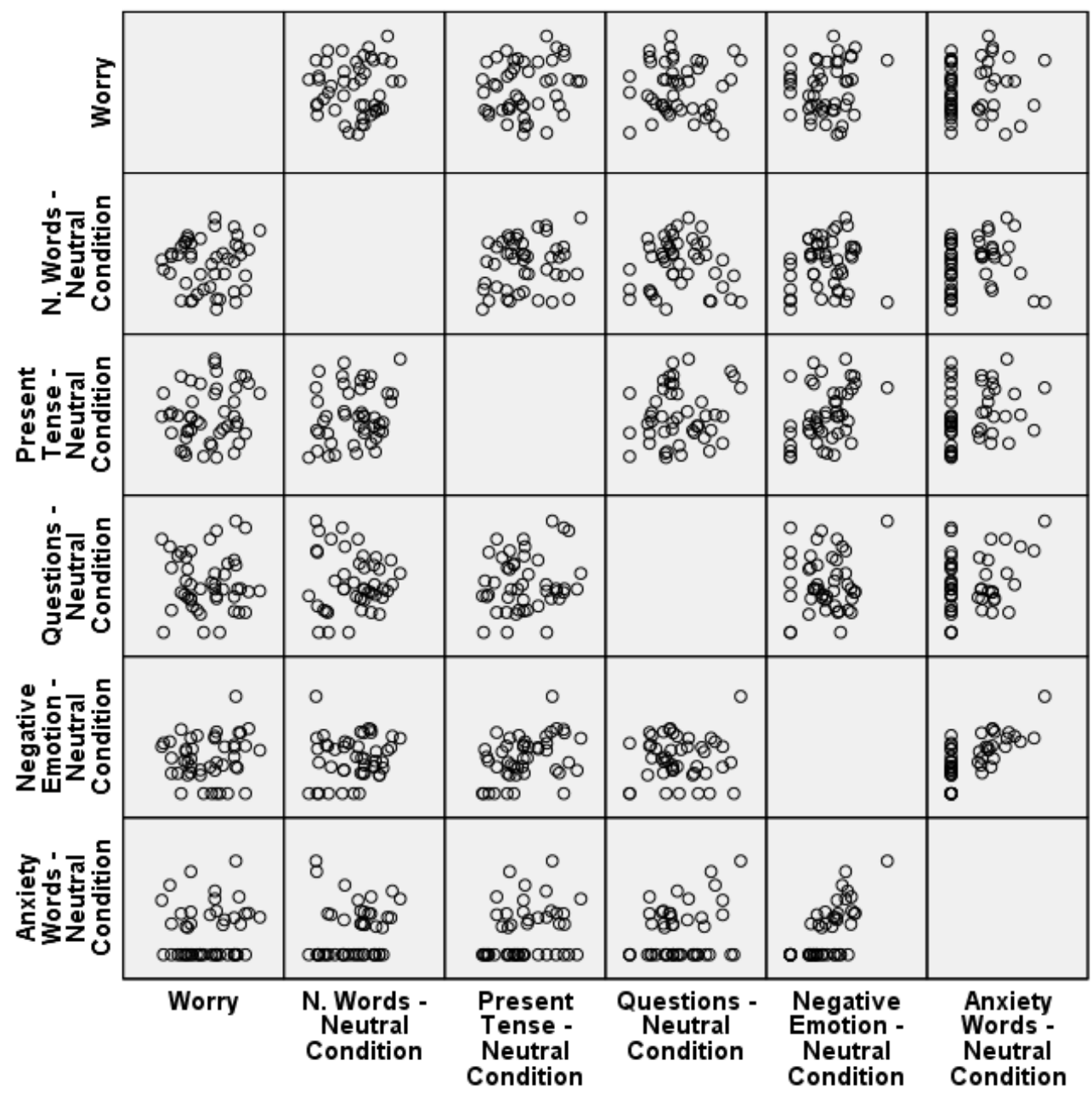

Figure 17 . Worry and language scatterplots - neutral condition. 


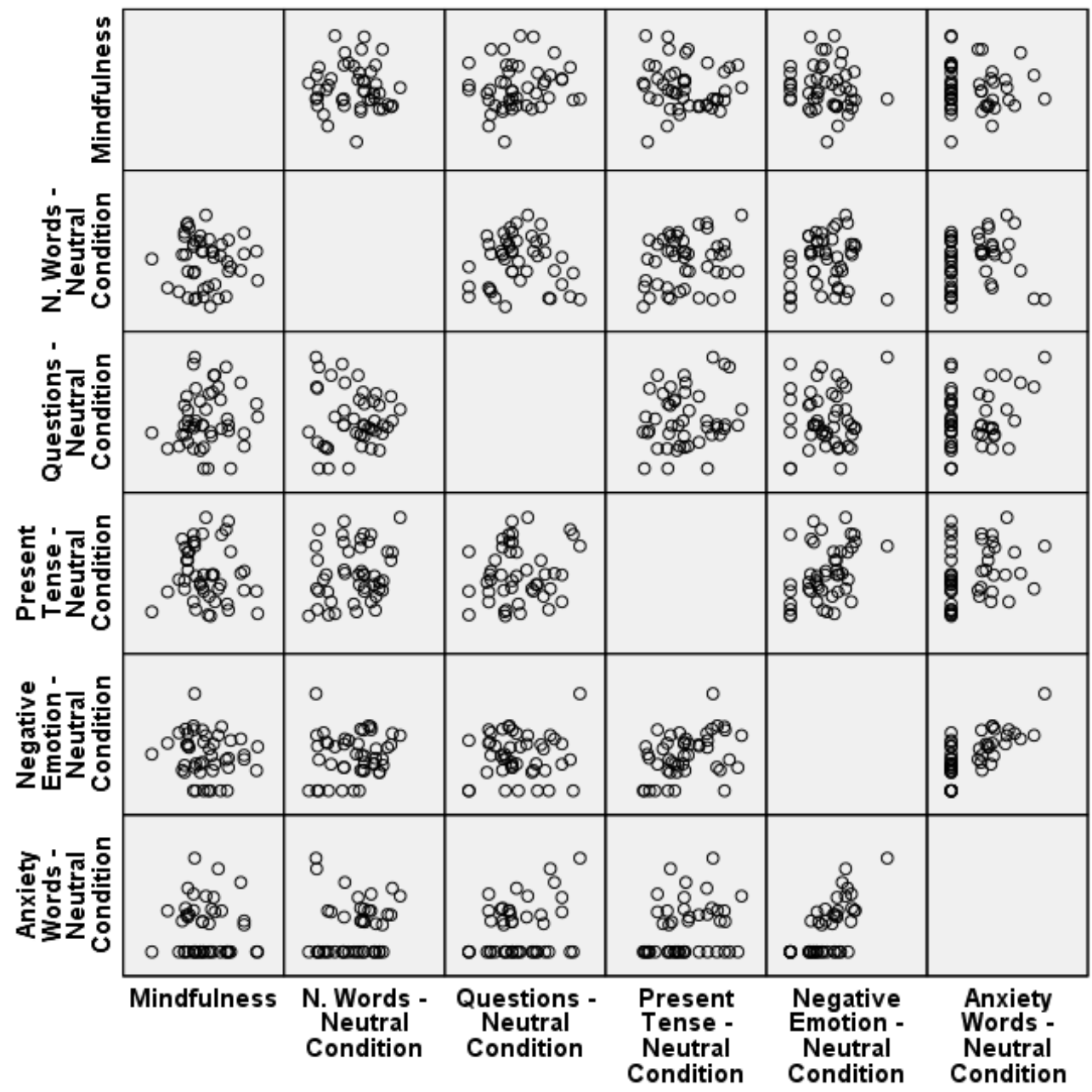

Figure 18. Mindfulness and language scatterplots - neutral condition. 


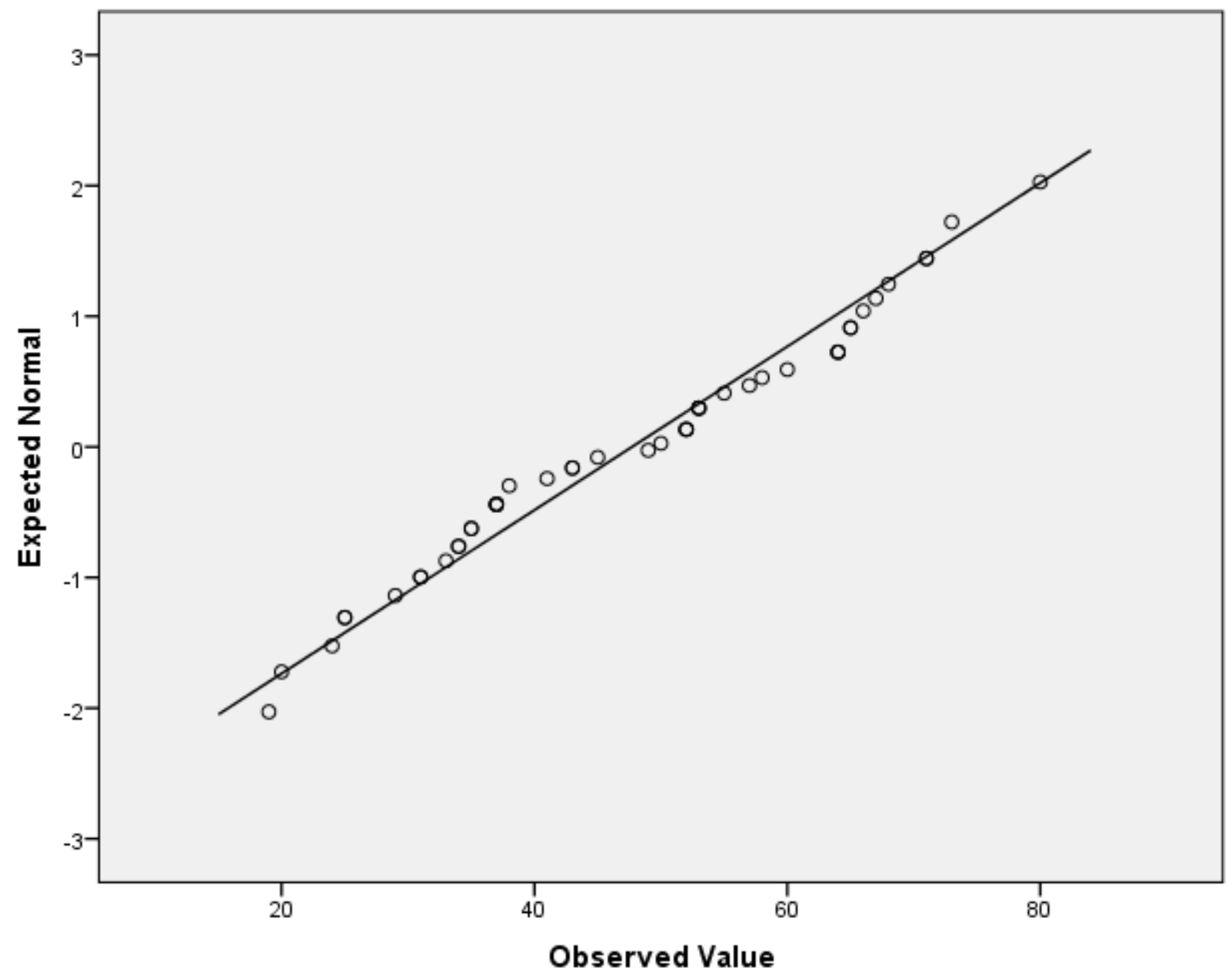

Figure 19. Worry Q-Q plot. 


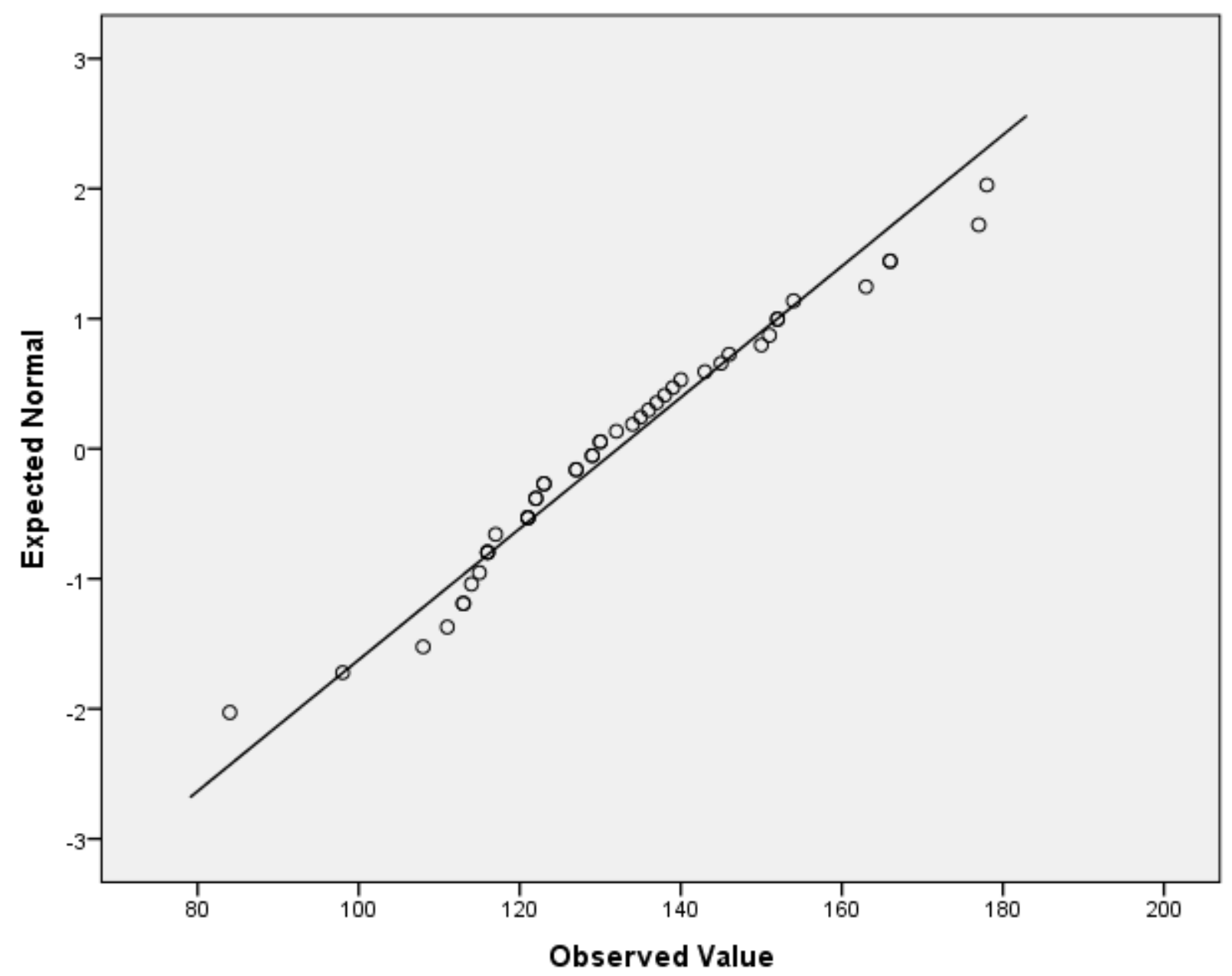

Figure 20. Mindfulness Q-Q plot. 


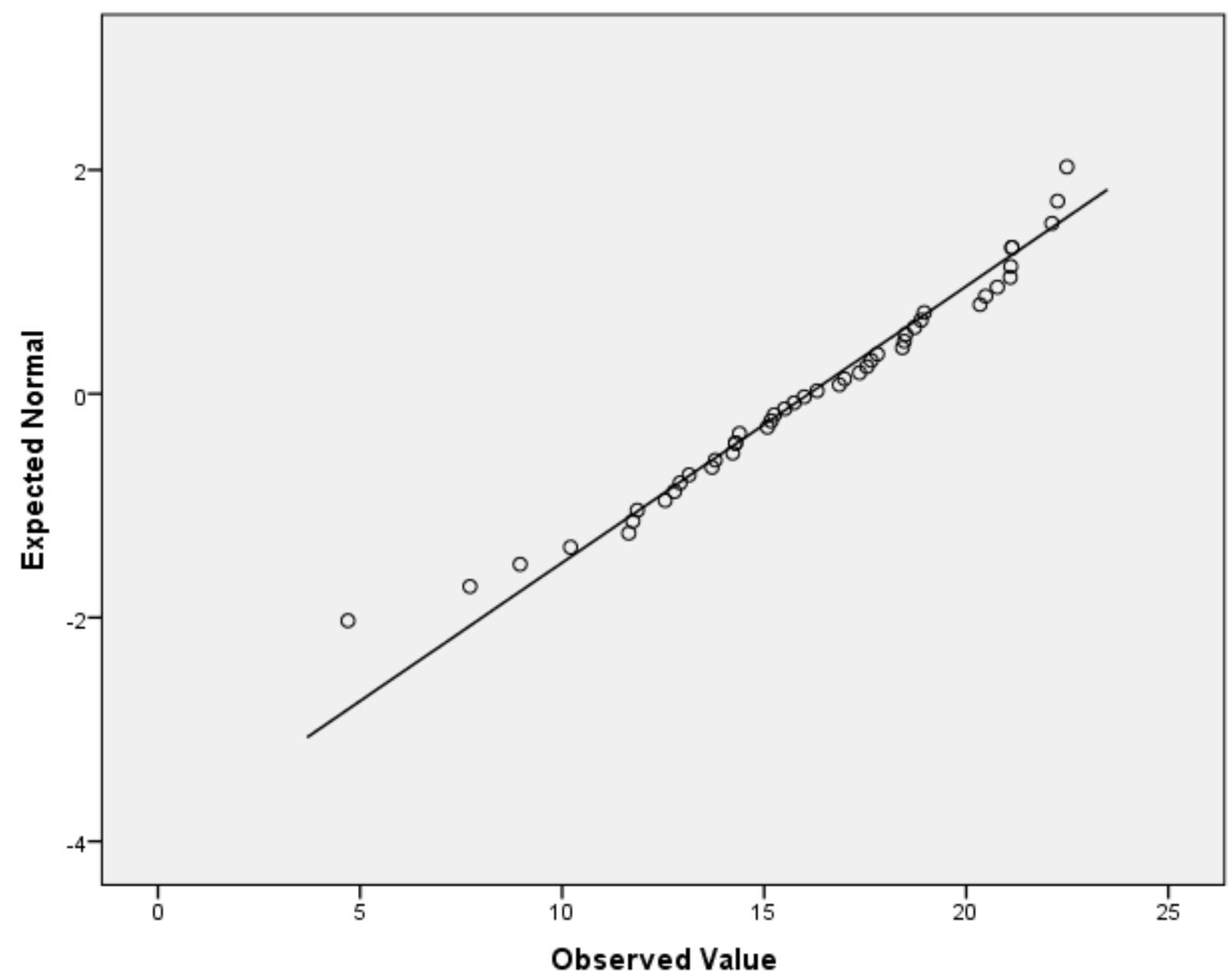

Figure 21. Present tense - worry condition Q-Q plot. 


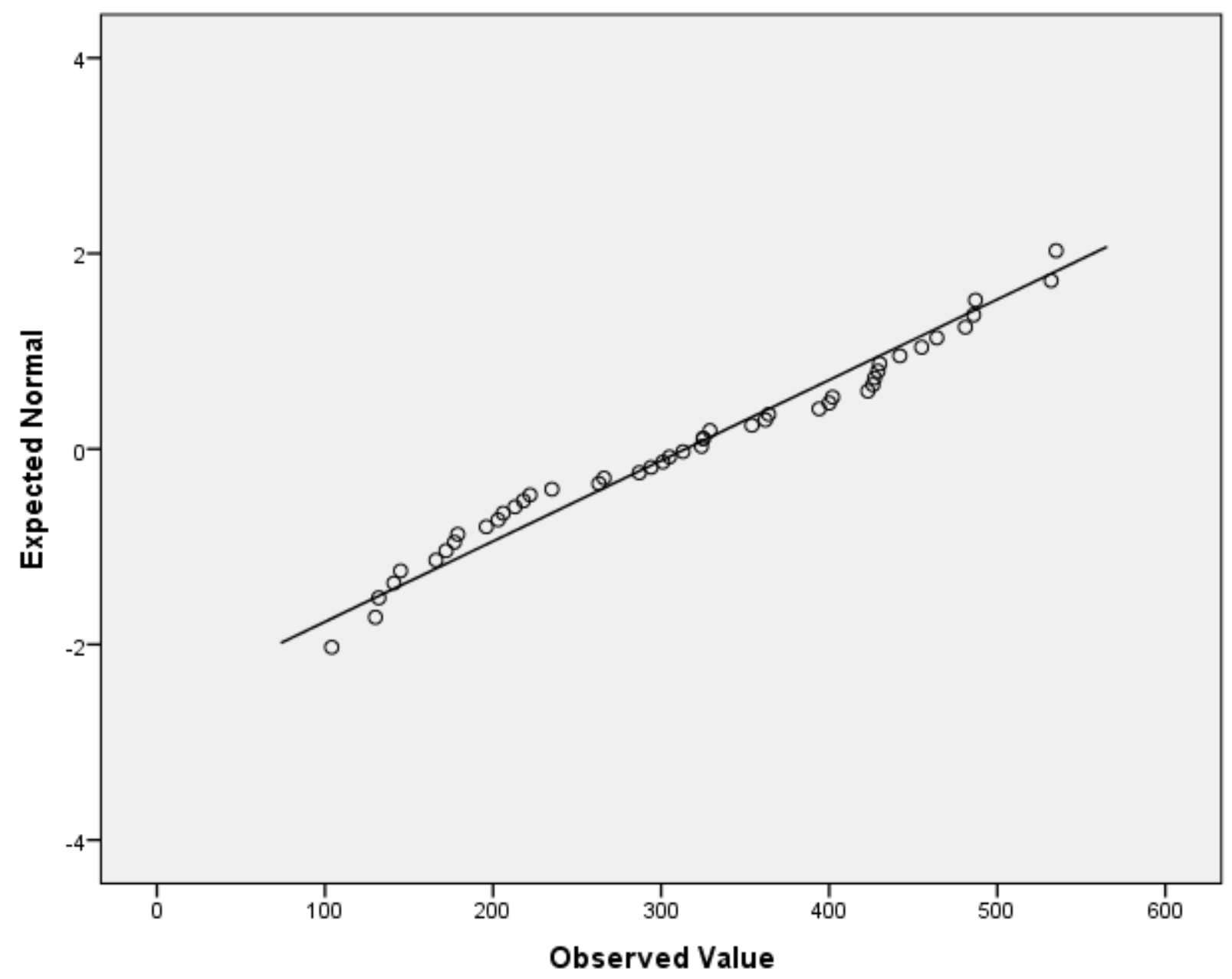

Figure 22. Number of words - worry condition Q-Q plot. 


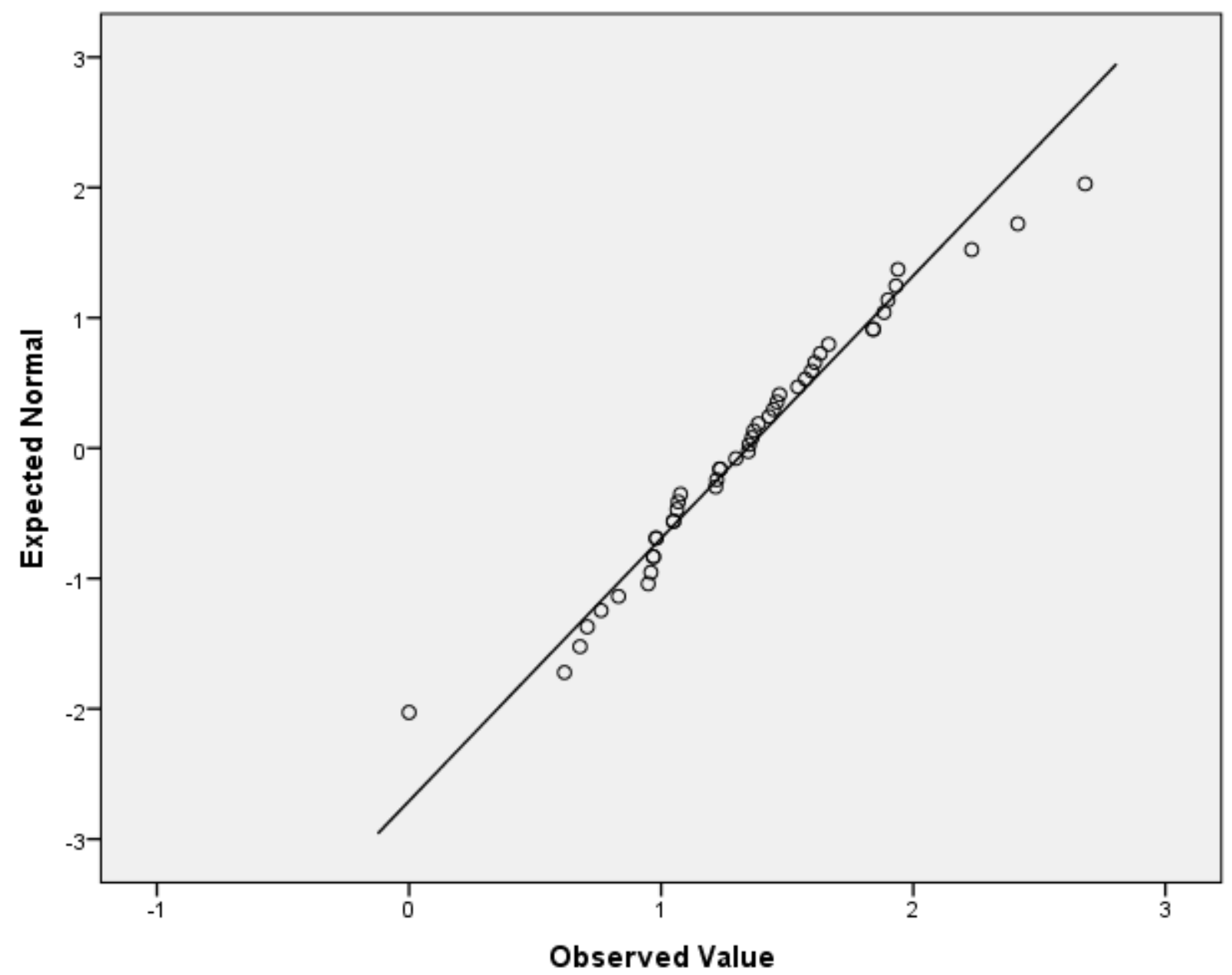

Figure 23.Questions - worry condition Q-Q plot. 


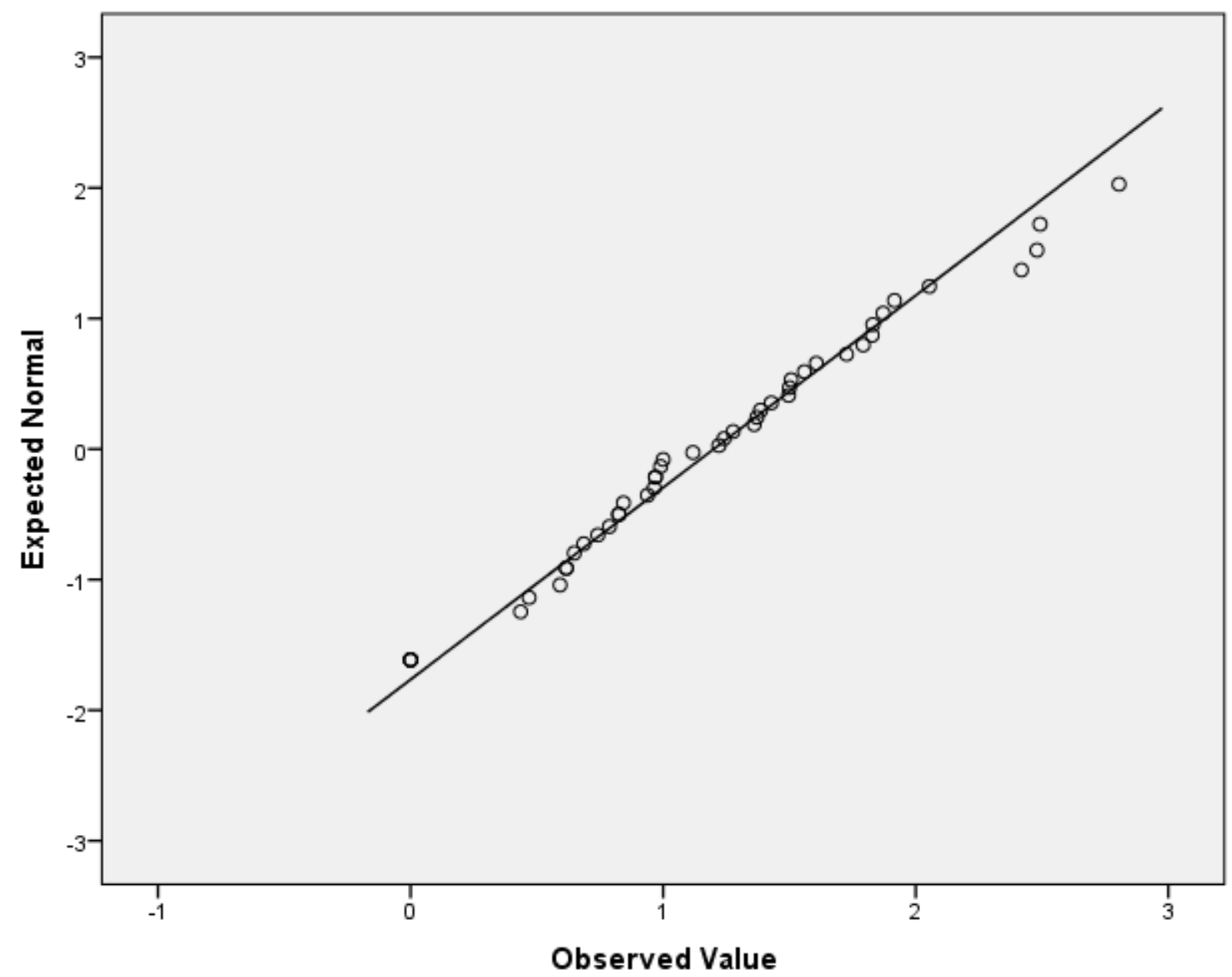

Figure 24. Anxiety related words - worry condition Q-Q plot. 


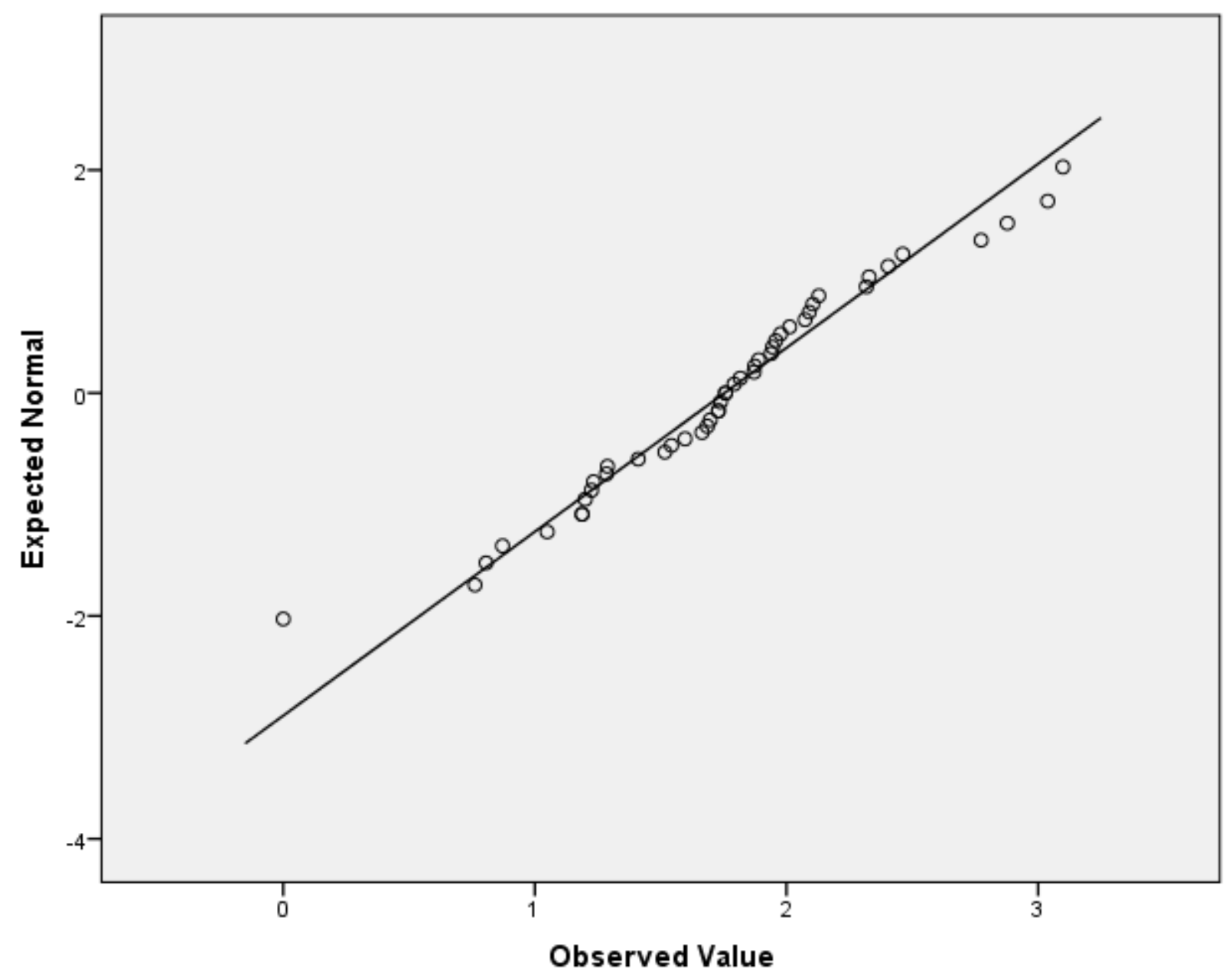

Figure 25. Negative emotion words - worry condition Q-Q plot. 


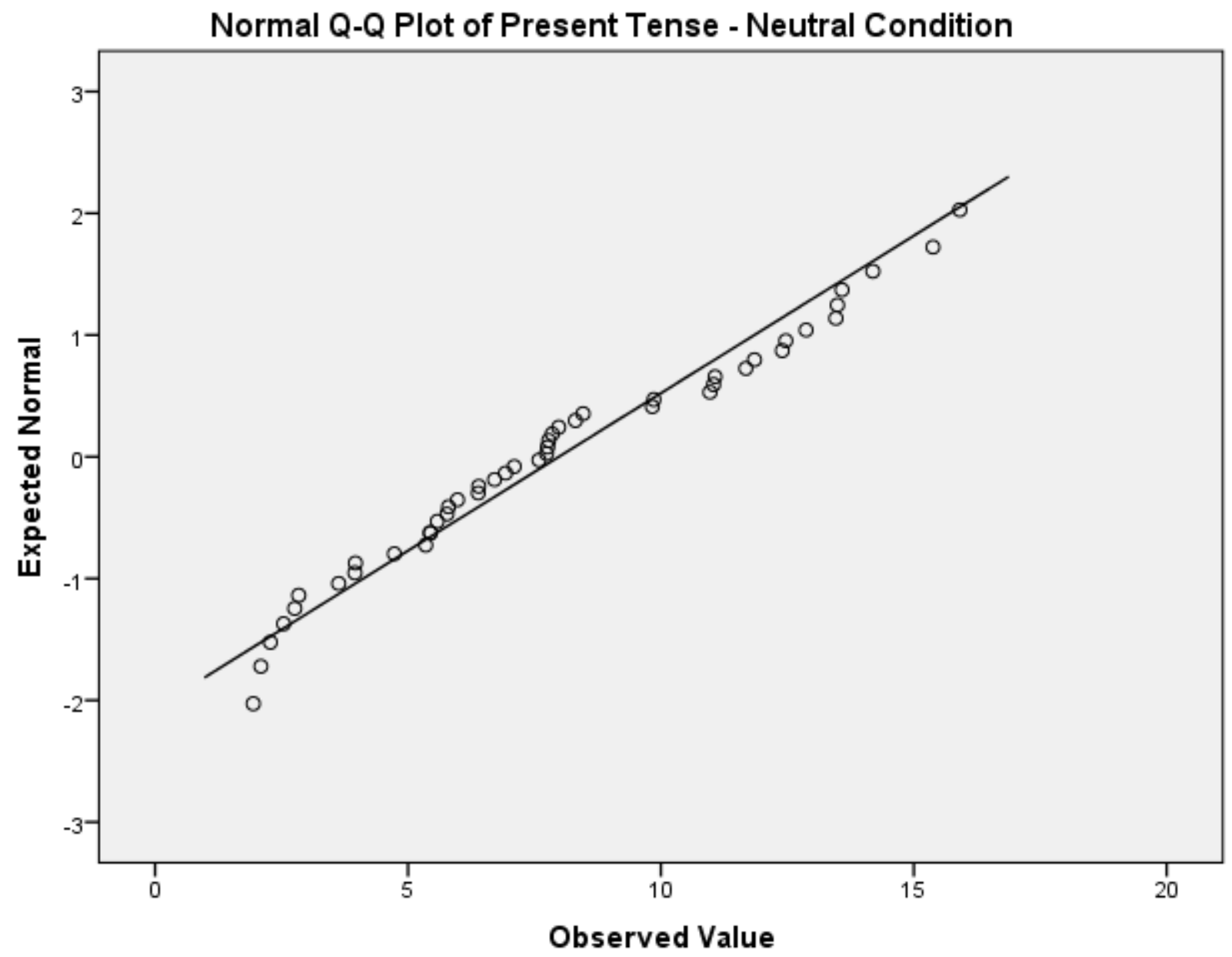

Figure 26. Present tense - neutral condition Q-Q plot. 


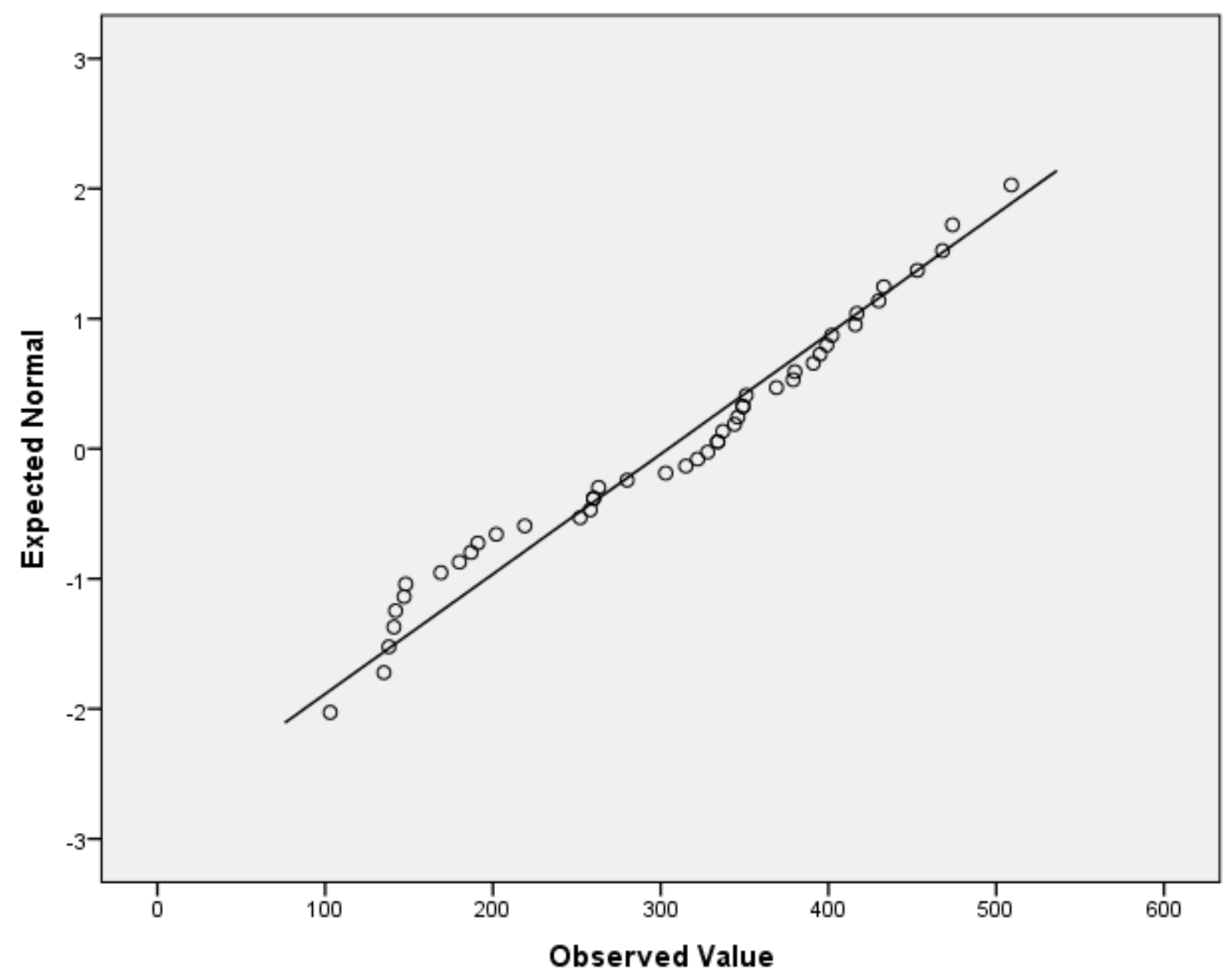

Figure 27. Number of words - neutral condition Q-Q plot. 


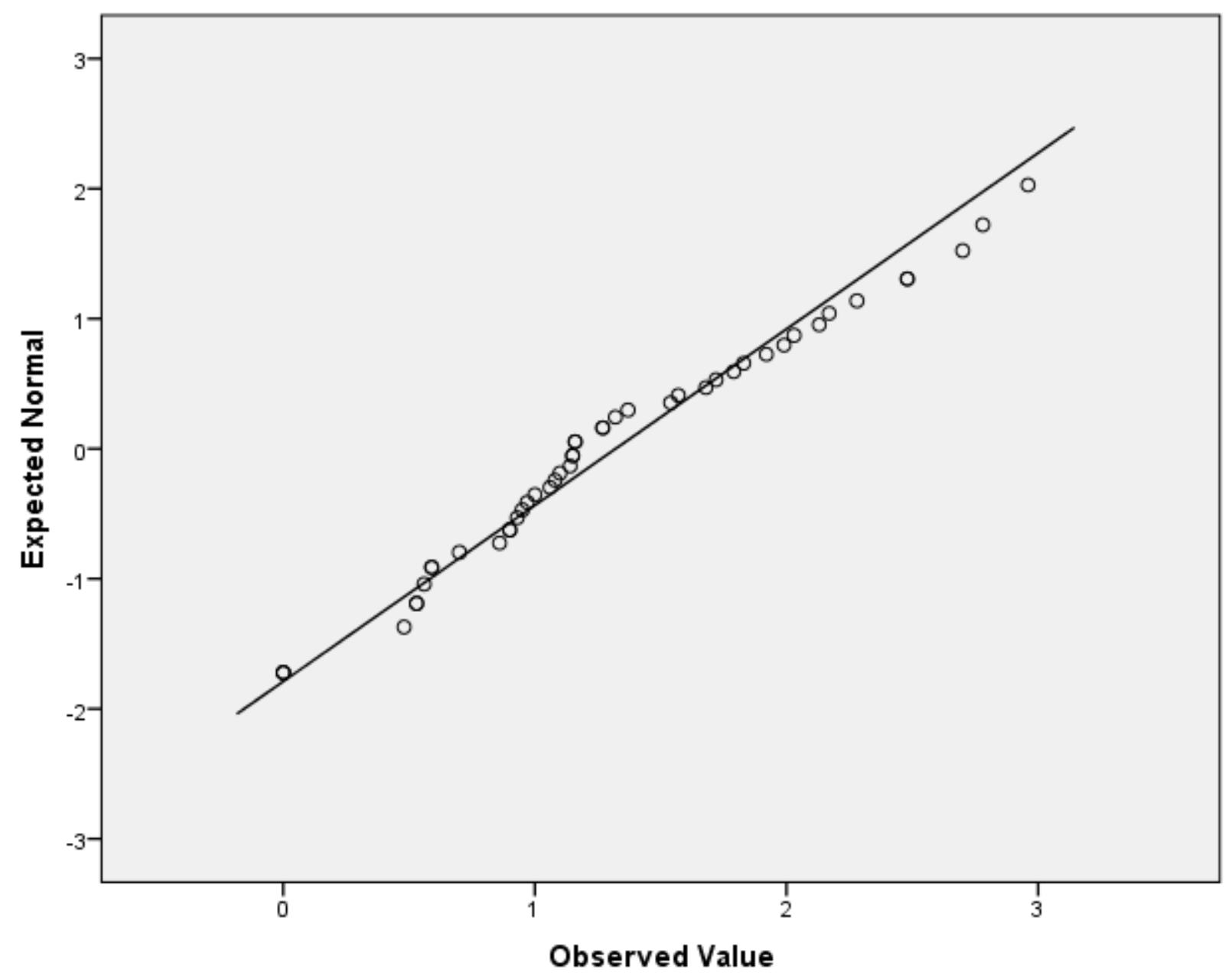

Figure 28. Number of questions - neutral condition Q-Q plot. 


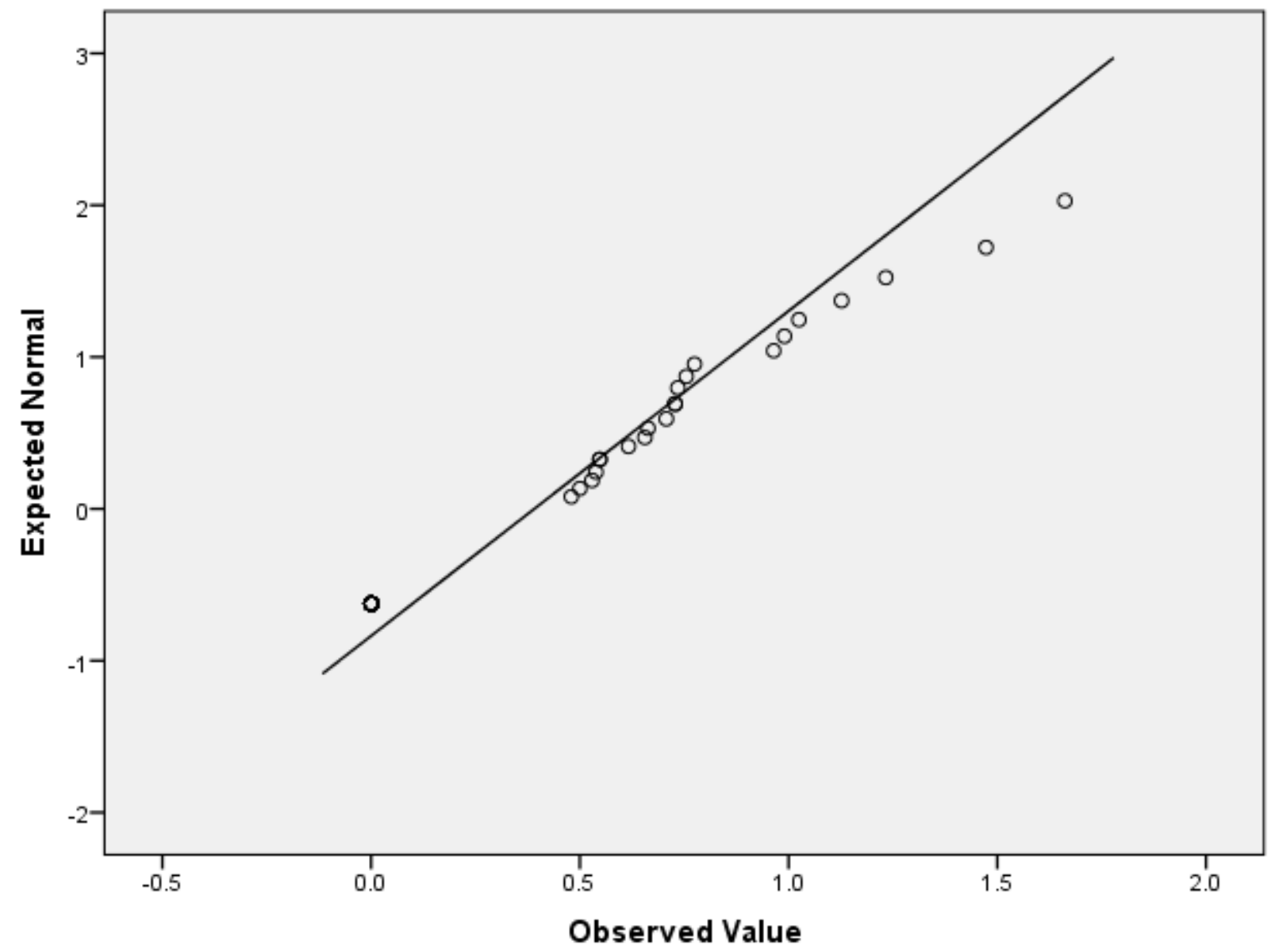

Figure 29. Anxiety related words - neutral condition Q-Q plot. 


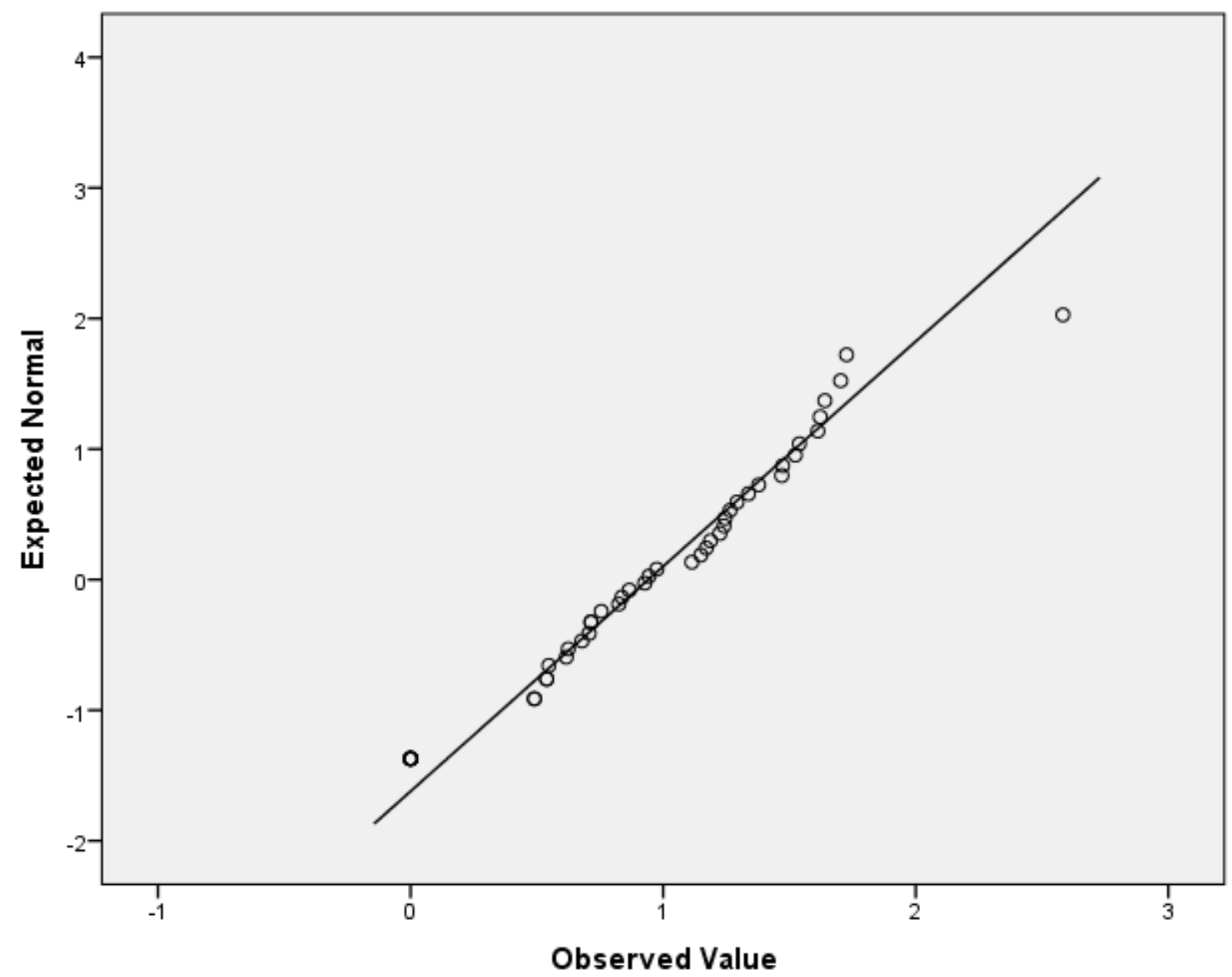

Figure 30. Negative emotion words - neutral condition Q-Q plot. 
APPENDIX I. 
Worry writing task instructions:

"What have you most worried about lately? Ok, now we are curious to know about what goes through your mind when you are worrying. Just before we start, please pretend you are re-experiencing the last time you worried about XXX. Close your eyes and imagine yourself in the last time you worried about XXX. Just let the thoughts about XXX naturally come to your mind (give participant $1 \mathrm{~min}$ ). Now what we would like you to do is to write the thoughts that go through your mind while you are worrying. Do not worry about any typing errors, or even making any sense, since worry is an internal, personal activity. You don't need to write exclusively about your main worry topic, you can write about anything that comes to your mind while you are worrying. So this is a brief example of the kinds of things you may write about: I am afraid my children will not have a good future, will their grades be good to get in a good University?- then the worries would continue to go on and on, like in a text. So what we are asking you to do is to basically "to transcribe the worries from your mind to the screen", is that clear? Do you have any questions? You will have plenty of time to complete this task. Please make sure you include a title in your writing."

Neutral writing task instructions:

"What was the most interesting thing you did in the last days? Ok, now we are interested in knowing what you think as you remember this event. Just before we start, please pretend you are experiencing that event again. Close your eyes and imagine yourself as you experienced that event. Just let the memories about that event come to your mind (give participant $1 \mathrm{mim}$ ). Now what we would like you to do is to write the 
thoughts that go through your mind while you think about that experience. Do not worry about any typing errors, or even making any sense, since this is just about your experience. You don't need to write only about [event], you can write about anything that comes to your mind as you recall it. So this is a brief example of the kinds of things you may write about: I think going to the party on Saturday was fun, what did we have for diner? - then the things you think of or remember would continue to go on and on, like in a text. So what we are asking you to do is to basically "write what you think about that event in the screen", is that clear? Do you have any questions? You will have plenty of time to complete this task. Please make sure you include a title in your writing." 


\section{CURRICULUM VITAE}

Elena M. C. Geronimi Bortoleto, M.A.

Department of Psychological and Brain Sciences

317 Life Sciences Building

University of Louisville

Louisville, KY 40292

\section{Education}

Ph.D. - Clinical Psychology, University of Louisville, expected December 2019 Dissertation Title: Understanding worry and mindfulness through psycholinguistics

Mentor: Janet Woodruff-Borden, Ph.D.

Master of Arts - Clinical Psychology, University of Louisville, 2014

Bachelor of Arts - Psychology Major, concentration in Natural and Social Sciences, University Louisville, 2011

Bachelor of Business Administration - Federal University of Rio de Janeiro, Brazil, 2004

\section{Languages}

Fluent in English, Spanish, French, Portuguese, and Italian

Study abroad experience, Lyon, France, 2004

Attended a semester of Business Administration in University Jean Moulin

Member of AECLA (Student Association for the Latin American Culture)

\section{Research Experience}

The Anxiety and Stress in Kids Lab

July 2012 - July 2018

University of Louisville, Louisville-KY

Mentor: Janet Woodruff-Borden, Ph.D.

Graduate Student Research Assistant

Activities include:

o Recruitment and running participants

o Data collection and management 
o Data analysis

o Manuscript preparation

o Supervision of post bachelor lab assistants

o Maintaining IRB approval (July 2012-June 2014)

The Anxiety and Stress in Kids Lab 2012

August 2010 - June

University of Louisville, Louisville-KY

Mentor: Janet Woodruff-Borden, Ph.D.

Volunteer Post-Baccalaureate Research Assistant

Responsibilities included:

o Recruited and ran participants

o Administered measures

o Coded and transcribed data

o Assisted with results analysis

o Assisted with manuscript preparation

\section{$\underline{\text { Publications }}$}

Geronimi, E. M. C., Richards, A, Szabo-Long, C., \& Woodruff-Borden, J. (2019). A preliminary investigation of cognitive features associated with worry among African American youth. The Journal of Black Psychology. doi: $10.1177 / 0095798419870076$

Geronimi, E. M. C., Arellano, B., \& Woodruff-Borden, J. (2019). Relating mindfulness and executive function in children. Clinical Child Psychology and Psychiatry. doi: 10.1177/1359104519833737

Gramszlo, C., Geronimi, E. M. C., Arellano, B., \& Woodruff-Borden, J. (2018). Testing a cognitive pathway between temperament and childhood anxiety. Journal of Child and Family Studies, 27(2), 580-590. doi: 10.1007/s10826-0170914-2

Geronimi, E. M. C., Patterson, H. L., \& Woodruff-Borden, J. (2016). Relating worry and executive functioning during childhood: The moderating role of age. Child Psychiatry \& Human Development, 47(3), 430-439. doi: 10.1007/s10578015-0577-4

Affrunti, N. W., Geronimi, E. M. C., \& Woodruff-Borden, J. (2015). Language of perfectionistic parents predicting child anxiety diagnostic status. Journal of Anxiety Disorders. doi: 10.1016/j.janxdis.2015.01.001

Geronimi, E. M. C., \& Woodruff-Borden, J. (2015). The language of worry: Examining linguistic elements of worry models. Cognition and Emotion, 29(1-2), 311-318. doi: 10.1080/02699931.2014.917071 
Affrunti, N. W., Geronimi, E. M. C., \& Woodruff-Borden, J. (2014).

Temperament, peer victimization, and nurturing parenting in child anxiety: A moderated mediation model. Child Psychiatry and Human Development, 45(4), 483-492.

doi: $10.1007 / \mathrm{s} 10578-013-0418-2$

Williams, S. R., Cash, E., Daup, M., Geronimi, E. M. C., Sephton, S. E., \& Woodruff-Borden, J. (2013). Exploring patterns in cortisol synchrony among anxious and nonanxious mother and child dyads: A preliminary study. Biological Psychology, 93, 287-295. doi: 10.1016/j.biopsycho.2013.02.015

\section{$\underline{\text { Presentations }}$}

Geronimi, E. M. C., \& Woodruff-Borden, J. (2018). Understanding the language of depression in late childhood and early adolescence. Poster presented at the Association for Behavioral and Cognitive Therapies Annual Convention, Washington, DC.

Geronimi, E. M. C., Arellano, B., \& Woodruff-Borden, J. (2017). Linking mindfulness and executive function in children: The mediating role of intolerance of uncertainty. Poster presented at the Association for Behavioral and Cognitive Therapies Annual Convention, San Diego, CA.

Geronimi, E. M. C., Gramzlo, C., Arellano, B., Affrunti, N. W., \& WoodruffBorden, J. (2016). Temperament and Mindfulness in Children: The Mediating Role of Intolerance of Uncertainty. Poster presented at the Association for Behavioral and Cognitive Therapies Annual Convention, New York, NY.

Affrunti, N.W., Geronimi, E.M.C., Gramszlo, C., Arellano, B. \& WoodruffBorden, J. (October, 2016). Negative affect, perfectionism, and intolerance of uncertainty in a model of risk for child anxiety diagnostic status. Poster presented at the Association for Behavioral and Cognitive Therapies Annual Convention, New York, NY.

Arellano, B., Affrunti, N. W., Geronimi, E. M. C., Gramszlo, C., \& WoodruffBorden, J. (2016). GAD severity predicts parenting behavior during a mildly stressful task. Poster presented at the Association for Behavioral and Cognitive Therapies Annual Convention, New York, NY.

Geronimi, E. M. C., Szabo-Long, C., Patterson, H., Affrunti, N.W., Richards, A., \& Woodruff-Borden, J. (2015). Understanding Worry in Adolescents: Linguistic Features of Theoretical Worry Models. Poster presented at the Association for Behavioral and Cognitive Therapies Annual Convention, Chicago, IL. 
Affrunti, N.W., Geronimi, E. M. C., Szabo-Long, C., Patterson, H., Richards, A., \& Woodruff-Borden, J. (2015). The effect of maternal psychopathology on parent-child agreement of child anxiety symptoms: A hierarchical linear modeling approach. Poster presented at the Association for Behavioral and Cognitive Therapies Annual Convention, Chicago, IL.

Affrunti, N.W., Geronimi, E. M. C., Szabo-Long, C., Patterson, H., Richards, A., \& Woodruff-Borden, J. (2015). Fearful temperament moderates the relation between perfectionism and child worry and anxiety. Poster presented at the Association for Behavioral and Cognitive Therapies Annual Convention, Chicago, IL.

Patterson, H., Affrunti, N., Geronimi, E. M. C., Richards, A., Szabo-Long, C. \& Woodruff Borden, J. (2015). Preliminary evidence for a physiological avoidance mechanism in children: comparison with adult research on heart rate variability and worry. Poster presented at the Association for Behavioral and Cognitive Therapies Annual Convention, Chicago, IL.

Szabo-Long, C., Richards, A., Geronimi, E. M. C., Affrunti, N., Patterson, H., \& Woodruff Borden, J. (2015). Ethnic identity moderates risk for worry in african american youth. Poster presented at the Association for Behavioral and Cognitive Therapies Annual Convention, Chicago, IL.

Geronimi, E. M. C., Affrunti, N. W., Meghan A. Jones, \& Woodruff-Borden, J. (2014). The language of overcontrolling parental behaviors. Poster presented at the Association for Behavioral and Cognitive Therapies Annual Convention, Philadelphia, PA.

Geronimi, E. M. C., Affrunti, N. W., Meghan A. Jones, \& Woodruff-Borden, J. (2014). Verb tense patterns in individuals with GAD versus controls: An indication of temporal bias. Poster presented at the Association for Behavioral and Cognitive Therapies Annual Convention, Philadelphia, PA.

Geronimi, E. M. C., Affrunti, N. W., Meghan A. Jones, \& Woodruff-Borden, J. (2014). Cardiac modulation as a predictor of worry in children. Poster presented at the Association for Behavioral and Cognitive Therapies Annual Convention, Philadelphia, PA.

Affrunti, N. W., Geronimi, E. M. C., \& Woodruff-Borden, J. (2014). Executive functioning mediates the relation between child perfectionism, anxiety, and worry. Poster presented at the Association for Behavioral and Cognitive Therapies Annual Convention, Philadelphia, PA.

Affrunti, N. W., Geronimi, E. M. C., \& Woodruff-Borden, J. (2014). Parent anxiety, perfectionism, and overcontrolling parenting in child anxiety. Poster presented in the ADAA Annual Conference in Chicago, IL. 
Affrunti, N. W., Geronimi, E. M. C., \& Woodruff-Borden, J. (2014). Family factors in the development, maintenance, and treatment for childhood anxiety. Symposium presented in the ADAA Annual Conference in Chicago, IL.

Geronimi, E. M. C., Affrunti, N. W., \& Woodruff-Borden, J. (2013).

Investigating linguistic features of GAD parents versus controls. Poster presented at the Association for Behavioral and Cognitive Therapies Annual Convention, Nashville, TN.

Geronimi, E. M. C., Affrunti, N. W., \& Woodruff-Borden, J. (2013). Linguistic expressions of worry models in individuals with GAD. Poster presented at the Association for Behavioral and Cognitive Therapies Annual Convention, Nashville, TN.

Affrunti, N. W., Geronimi, E. M. C., \& Woodruff-Borden, J. (2013). The influence of parent and child perfectionism on child anxiety and worry. Poster presented at the Association for Behavioral and Cognitive Therapies Annual Convention, Nashville, TN.

Affrunti, N. W., Geronimi, E. M. C., \& Woodruff-Borden, J. (2013). The association of executive function and temperament in a model of risk for childhood anxiety. Poster presented at the Association for Behavioral and Cognitive Therapies Annual Convention, Nashville, TN.

Geronimi, E. M. C., Williams, S. R., Carrico, C., Kertz, S. J., \& WoodruffBorden, J. (2012). Autonomic responsiveness, intolerance of uncertainty and behavior control in child worry and anxiety. Poster presented at the Association for Behavioral and Cognitive Therapies Annual Convention, National Harbor, MD.

Williams, S. R., Geronimi E. M. C., Carrico, C., \& Woodruff-Borden, J. (2012). Physiological symptoms of anxiety, family functioning, and parent factors as predictors of salivary cortisol diurnal slope in childhood. Poster presented at the Association for Behavioral and Cognitive Therapies Annual Convention, National Harbor, MD.

Geronimi, E. M. C., Williams, S. R., Kertz, S. J., \& Woodruff-Borden, J. (2011). Examining the autonomic responsiveness of children with high and low worry to threat and nonthreat pictures. Poster presented in the 2011 Undergraduate Research Symposium at the University of Louisville.

Geronimi, E. M. C., Williams, S. R., Kertz, S. J., Schrock, M., Hosey, R., \& Woodruff-Borden, J. (2011). Examining the autonomic responsiveness of children with high and low worry to threat and nonthreat pictures. Poster presented at the 
Association for Behavioral and Cognitive Therapies Annual Convention, Toronto, Canada.

\section{Clinical Experience}

\section{Internship}

Children and Adolescent Outpatient Services, Hutchings Psychiatric Center, Syracuse- $N Y$

Superisor: Kristyn Saveliev, Ph.D. September 2018 - August 2019

Responsibilities include:

o Providing evidence based treatments (individual and group modalities) to a population of children and adolescents experiencing a wide range of diagnoses.

o Evaluations pertaining a number of domains including, learning disability, and several psychological disorders (e.g., anxiety; depression; bipolar disorder)

Adult Inpatient Services (Treatment Unit One), Hutchings Psychiatric Center, Syracuse- $N Y$

Superisor: Steve Moore, Psy.D. September 2018 - August

2019

Responsibilities include:

o Providing evidence based treatments (individual and group modalities) to a population of adults experiencing a wide range of diagnoses.

o Evaluations pertaining several psychological disorders (e.g., depression; bipolar disorder; schizophrenia)

Children and Adolescent Inpatient Services Hutchings Psychiatric Center, Syracuse-NY

Superisor: Carl Vondensteinen, Ph.D. $\quad$ February 2019 - August 2019 Responsibilities include:

o Co-leading a process group with children presenting with different diagnoses and conditions (e.g., ODD; Depression; severe suiciadality) and backgrounds.

\section{Clinical Practica}

Integrative Intervention Team, Psychological Services Center, University of Louisville, Louisville-KY

Supervisor: Richard Lewine, Ph.D. January 2017 - May 2018

Responsibilities include: 
o Providing integrative treatment to a population of adults experiencing a wide range of diagnoses from anxiety and depressive disorders to bipolar and personality disorders

o Attending weekly supervision meetings

o Peer supervision

o Peer observation

Strong Minds: Child and Adolescent Psychology Specialists, Private Practice, Louisville-KY

Supervisor: Tanya Stockhammer, Ph.D. January 2017 - May 2018

Responsibilities include:

o Evidence-based treatment to children and adolescents, and their families

o School consultation

o Weekly supervision meetings

Children with ADHD and Related Difficulties Treatment Team, Psychological Services Center, University of Louisville, Louisville-KY

Supervisor: Paul Rosen, Ph.D. September 2015 - March 2016

Responsibilities include:

o Evidence-based assessment and treatment to children and adolescents, and their families

o School consultation

o Client reports

o Weekly supervision meetings

o Peer supervision

o Peer observation

Mindfulness Interventions Treatment Team, Psychological Services Center, University of Louisville, Louisville-KY

Supervisor: Paul Salmon, Ph.D. September 2014 - August

2015

Responsibilities include:

o Providing mindfulness-based treatment to a population of adults experiencing a wide range of anxiety and mood disorders

o Attending weekly supervision meetings

o Peer supervision

o Peer observation

Cognitive-Behavioral Therapy for Anxiety Disorders Treatment Team, Psychological Services Center, University of Louisville, Louisville-KY

Supervisor: Janet Woodruff-Borden, Ph.D. July 2012 - August 2014

Responsibilities include:

o Providing cognitive behavioral treatment to a population of adults 
experiencing a wide range of anxiety and mood disorders

o Attending weekly supervision meetings

o Peer supervision

o Peer observation

Anxiety Disorders Diagnostic Interviews, The Anxiety and Stress in Kids Lab, Psychological Services Center, University of Louisville, Louisville-KY

Supervisor: Janet Woodruff-Borden, Ph.D. July 2014 - Present

Responsibilities include:

o Administering the Anxiety Disorders Interview Schedule for DSM-IV and DSM -V; client and parent/child versions

o Writing feedback reports

Assessment Practicum, Psychological Services Center, University of Louisville, Louisville-KY

Supervisors: Bernadette Walter, Ph.D. \& David Winsch, Ph.D.

Responsibilities include:

July 2012 - May 2018

o Evaluations pertaining a number of domains including, advanced placement, learning disability, and several psychological disorders (e.g., anxiety, depression, ADHD) for children and adults

o Standardized test administration, scoring, and interpretation

o Preparation of integrated reports

o Client feedback

\section{Grants}

Geronimi, E. M. C., Szabo-Long, C. M., Affrunti, N. W., Woodruff-Borden, J. (2016). Understanding the Nature of Worry through Linguistic Patterns. Graduate Student Council's Spring 2016 Research Grant. Amount requested \$300 (funded)

Geronimi, E. M. C. (2011). Children autonomic responses to threat and nonthreat stimuli. Amber M. Heuerman Undergraduate Honors Research Fund. Amount requested: $\$ 500.00$ (funded)

Geronimi, E. M. C. (2011). Examining the autonomic responsiveness of children with high and low worry to threat and nonthreat pictures. Undergraduate Research Scholar Grant-Vice President for Research. Amount requested: $\$ 275.00$ (funded)

\section{Honors}

Geronimi, E. M. C. (July 2014 - June 2016) Fellowship awarded by the School of Interdisciplinary and Graduate Studies, University of Louisville 
Psychology Honors Thesis - “Autonomic Inflexibility in Children”, faculty mentor - Janet Woodruff-Borden, Ph.D., Developmental Psychopathology Laboratory, University of Louisville, 2011

\section{Employment History}

Teaching Assistantship - University of Louisville / Jan. 2017 - May 2018

o Assisting with Psychology undergraduate lectures

o Grading exams and assignments

o Offering office hours for questions on the content, reviewing exams, etc.

o Proctoring exams

People Planning and Development Analyst - Zilor / Jul. 2005 - Jan. 2010

Managed installation of SAP (System Analysis and Program Development) software - a managerial tool that was installed in all HR areas

Acted on different HR processes, including:

o Developed training programs for different areas

o Recruited and selected new employees

o Designed compensation policies and acted on its processes

o Created organization chart modifications and adaptations

\section{Italian Teacher - CNI Language School / Feb. - Dec. 2006}

Taught in the Acceleration Program, an intensive languages program designed for people who want to learn quickly and effectively

\section{Purchase Project Management Intern - “Peugeot Citroën” / Apr. - Dec. 2003}

o Coordinated new automobile pieces implementation and technical modifications

o Administrated the purchase systems

o Represented the Project Management Department in weekly interdisciplinary meetings with Quality, Logistics, Engineering and Plant employees

o Participated in the elaboration, development, and implementation of "Gestion" system that managed all projects in progress in the Purchase Department

Finance Intern - “Unimed Rio” / Aug. - Dec. 2002

o Analyzed and monitored the company's budget

o Conducted profitability comparisons among the suppliers

o Elaborated managerial expenses reports 